\title{
The way to understanding Chronic Postsurgical Pain
}

Citation for published version (APA):

Hoofwijk, D. M. N. (2018). The way to understanding Chronic Postsurgical Pain: From clinical and psychological predictors to incorporating genetics. [Doctoral Thesis, Maastricht University]. Datawyse / Universitaire Pers Maastricht. https://doi.org/10.26481/dis.20180905dh

Document status and date:

Published: 01/01/2018

DOI:

$10.26481 /$ dis.20180905dh

Document Version:

Publisher's PDF, also known as Version of record

\section{Please check the document version of this publication:}

- A submitted manuscript is the version of the article upon submission and before peer-review. There can be important differences between the submitted version and the official published version of record.

People interested in the research are advised to contact the author for the final version of the publication, or visit the DOI to the publisher's website.

- The final author version and the galley proof are versions of the publication after peer review.

- The final published version features the final layout of the paper including the volume, issue and page numbers.

Link to publication

\footnotetext{
General rights rights.

- You may freely distribute the URL identifying the publication in the public portal. please follow below link for the End User Agreement:

www.umlib.nl/taverne-license

Take down policy

If you believe that this document breaches copyright please contact us at:

repository@maastrichtuniversity.nl

providing details and we will investigate your claim.
}

Copyright and moral rights for the publications made accessible in the public portal are retained by the authors and/or other copyright owners and it is a condition of accessing publications that users recognise and abide by the legal requirements associated with these

- Users may download and print one copy of any publication from the public portal for the purpose of private study or research.

- You may not further distribute the material or use it for any profit-making activity or commercial gain

If the publication is distributed under the terms of Article $25 \mathrm{fa}$ of the Dutch Copyright Act, indicated by the "Taverne" license above, 

The way to understanding

\title{
Chronic Postsurgical Pain
}

\author{
From clinical and psychological predictors \\ to incorporating genetics
}

\author{
PROEFSCHRIFT \\ ter verkrijging van de graad van doctor aan de Universiteit Maastricht, \\ op gezag van de Rector Magnificus, \\ Prof. Dr. Rianne M. Letschert \\ volgens het besluit van het College van Decanen, \\ in het openbaar te verdedigen \\ op woensdag 5 september 2018 om 16.00 uur \\ door \\ Daisy M. N. Hoofwijk, \\ Geboren op 31 juli 1985, Sittard, Nederland
}




\section{Promotores}

Prof. Dr. W. F. F. A. Buhre

Prof. Dr. E. A. J. Joosten

\section{Co-promotores}

Dr. H.-F. Gramke

Dr. A. A. A. Fiddelers

\section{Beoordelingscommissie}

Prof. Dr. C. G. Faber (voorzitter)

Prof. Dr. I. C. Heyligers

Prof. Dr. R. F. P. M. Kruitwagen

Prof. Dr. G.J. Scheffer (Radboud UMC, Nijmegen)

Dr. M. P. Buise (Catharina Ziekenhuis, Eindhoven) 


\section{Contents}

Chapter 1 General introduction

Chapter 2 Prevalence and predictive factors of chronic postsurgical pain and poor global recovery one year after outpatient surgery

Chapter 3 Prevalence and predictive factors of chronic postsurgical pain and global surgical recovery one year after outpatient knee arthroscopy;

a prospective cohort study

Chapter 4 Severity of preoperative pain is associated with the amount of postoperative hospital-based health care costs during the first year after outpatient surgery

Chapter 5 Genetic polymorphisms and their association with the prevalence and severity of chronic postsurgical pain - A systematic review

Chapter 6 Genetic polymorphisms and prediction of chronic postsurgical pain 3 and 12 months after hysterectomy

Chapter 7 General discussion

Chapter 8 Summary

Samenvatting

Chapter 9 Valorisation addendum

Chapter 10 Acknowledgements Dankwoord

Chapter 11 Curriculum Vitae

List of publications 

Chapter

General introduction 



\section{General introduction}

In the Netherlands, approximately one and a half million surgical procedures are performed each year, in a population of 17 million inhabitants. ${ }^{1}$ Over $50 \%$ of these procedures are relatively minor and take place in an ambulatory setting. ${ }^{1}$ Even though many procedures are performed for life threatening disease (such as malignancy or severe cardiovascular disease), a large proportion of the surgical procedures are performed in order to improve the patient's quality of life ( $Q \circ \mathrm{L})$, by aiming to treat symptoms such as pain or functional impairment. ${ }^{1}$ Examples of surgical procedures to improve patients' QoL are orthopaedic procedures (e.g. knee arthroscopy, joint replacement), ENT-procedures (e.g. removal of tonsils, cochlear implant), neurosurgical procedures (e.g. discectomy) or general surgical procedures (e.g. hemorrhoidectomy, varicose vein surgery).

Many patients know and accept the fact that the acute postoperative phase comes with side effects such as postsurgical pain and diminished functional reserves, which results in a decreased QoL and a decreased capacity to participate in society. ${ }^{2,3}$

On the other hand, many patients and health care providers do not realise that these acute postsurgical effects may eventually result into long term side effects, as for instance chronic postsurgical pain (CPSP), and then may have major impact on life and society.

\section{Acute postsurgical pain (APSP)}

Research has shown that acute pain after surgery is still a serious problem. ${ }^{2-4}$ It is estimated that over $80 \%$ of all surgical patients experiences not adequately managed pain during the acute postoperative phase. ${ }^{4}$ Negative consequences of APSP have been clearly demonstrated as postoperative pain, along with nausea and vomiting, is one of the most important reasons for delayed hospital discharge, for contact with the general practitioner, and for unanticipated hospital admission. ${ }^{4,5}$ Furthermore, APSP causes discomfort and suffering. This implies that adequate pain management has a moral ground and is an essential part of optimal patient care. For this reason, the effectiveness of postoperative pain management is used as a quality indicator in Dutch hospitals. ${ }^{6}$ APSP is also associated with increased perioperative morbidity and mortality (e.g. myocardial infarction, stroke pulmonary infections, delirium). 4, 7, 8 Another important consequence of APSP is an increased risk of developing CPSP., ${ }^{9,10}$

The prevalence of APSP varies with the type of surgery performed. ${ }^{2}{ }^{11}$ Inpatient surgery is often more extensive than outpatient surgery, but more frequent pain measurements are possible, and more effective treatment techniques are available (e.g. epidural and neural catheter techniques, patient controlled analgesia). Acute pain treatment after outpatient surgery is more of a challenge and even though procedures are usually less extensive than inpatient procedures, research has shown that 
outpatient procedures are not always less painful. ${ }^{11}$ Some outpatient procedures, such as tonsillectomy and hemorrhoidectomy, are ranked amongst the most painful of all surgical procedures in the very large German 'Quality Improvement in Postoperative Pain Treatment' (i.e. QUIPS) cohort. ${ }^{11}$ During the last decades, multimodal analgesia techniques (such as loco-regional techniques) and safer analgesics (e.g. combined weak opioid analgesics) have become available for the treatment of pain after outpatient surgery, but has so far not resulted in a significant decrease in the prevalence of acute postsurgical pain. ${ }^{4}$

\section{Chronic postsurgical pain (CPSP)}

Initially, a definition for CPSP was proposed by Macrae et al. CPSP was defined as pain persisting for more than two months after surgery, and other possible causes for the pain should be excluded (e.g. malignancy, infection). ${ }^{12}$ According to this definition it was particularly important to exclude the possibility that the persistent pain was a continuation of pre-existing pain. ${ }^{12}$ Because it proved especially difficult to exclude preexisting pain as a possible cause for CPSP, a refined updated definition has recently been proposed by Werner et al. ${ }^{13}$ Pre-existing pain is no longer an exclusion criterion, but the CPSP has to be of increased intensity, and is preferably accompanied by a change in location or characteristic of the pain. ${ }^{13}$ Research has demonstrated that 10 $50 \%$ of all surgical patients report CPSP, ${ }^{9,12,14,15}$ and in $2-10 \%$ of the cases the reported pain is severe. 9

The transition from acute to chronic postsurgical pain is a complex process, of which the exact mechanisms are not yet fully understood. Based on the available theories, neurobiological changes in the nociceptive network which are induced during acute postoperative pain play a crucial role. ${ }^{9,16,17}$ Surgery and tissue damage lead to an increase in the release of inflammatory mediators at the level of peripheral nociceptors. ${ }^{9}$ This activates intracellular signalling pathways, which leads to a reduced threshold of these peripheral nociceptors and causes hypersensitivity (i.e. peripheral sensitisation). As surgery often causes nerve damage a neuropathic component is likely to be involved as well. Damaged nerves can produce persistent maladaptive plasticity, which finally results in ectopic firing of the remaining axonal sprouts. ${ }^{9,} 18$ Both the increased input of peripheral nociceptors, as well as the continued ectopic firing, will lead to an increased activation of central pain neurons in the spinal dorsal horn and the threshold of the so-called Pain Gate is decreased. ${ }^{19}$

This causes altered gene transcription of dorsal horn central sensory pain neurons, and this in turn leads to long-lasting more structural changes of these cells and their connections. ${ }^{9,20}$ Ectopic firing not only affects the central pain neurons in the dorsal horn, but may also induce the loss of neurons, especially the inhibitory interneurons, which are known to play a pivotal role in the regulation (i.e. opening and closing) of the spinal Pain Gate. ${ }^{9,16}$ Microglia are activated in the spinal cord and produce increased 
excitatory signalling in the dorsal horn. ${ }^{9,21,22}$ All these changes in the central nervous system are responsible for the process of central sensitisation, as observed in patients with CPSP. Besides changes at the level of the peripheral nerve and at the spinal cord Pain Gate, changes in function, structure and connectivity are also observed in supraspinal areas like the cortex and cortical grey matter. ${ }^{17}$ As there is no one to one relations between the duration and intensity of APSP and the development of CPSP, other mechanisms must play a role as well.

\section{Prediction of CPSP}

Researchers have attempted to unravel the risk factors or predictors for the development of CPSP. Since CPSP is associated with negative consequences such as patient suffering and high costs for society (e.g. absenteeism from work, health care costs), it is very important to identify these predictors. ${ }^{23,24}$ Especially risk factors that can be identified in the preoperative phase are of importance, since this might be able to offer targets for prevention of perioperative peripheral and central sensitisation processes. Research on predictive factors for CPSP has thus far focused on inpatients undergoing major surgery (e.g. cardiac surgery, thoracotomy, joint replacement, caesarean section, etc.). ${ }^{9,25-27}$

Among the clinical predictive factors, preoperative pain appears to be one of the most important predictors for the development of CPSP. ${ }^{10,15,28-30}$ As is the case with APSP, preoperative pain might lead to peripheral and central sensitization, which leaves the nervous system vulnerable for new pain inducing stimuli. ${ }^{15}$ Other described risk factors for the development of CPSP are type of surgery, ${ }^{10}$ surgical technique, ${ }^{9}, 10,31,32$ younger age, ${ }^{30,31}$ and female gender. 9,33

As psychosocial factors have proven to play an important role in chronic pain in general, ${ }^{34}$ the association between psychological risk factors and CPSP has also been extensively studied. Anxiety and more specifically fear for the surgical procedure (i.e. surgical fear), as well as pain catastrophizing have proven to be important risk factors for the development of CPSP. ${ }^{9,28,35,36}$ Other psychological factors, such as depression, pessimism and expectations of pain have also been correlated to CPSP. ${ }^{9}, 10$ More recently is has been suggested that these psychological variables are often correlated to each other, which is why a more general psychological composite variable, such as 'psychological robustness' results in a stronger association with CPSP than all other psychological variables separately. ${ }^{37,38}$

Unfortunately, most research in the field of CPSP only focused on demographic, clinical, and psychological predictors has not been able to explain all the variance observed in the prevalence and severity of CPSP. For this reason, genetic factors have become of interest to further improve prediction models which may then allow to better identify patients at risk. 


\section{Genetics}

Data based on twin studies and human pedigrees estimate that the heritability of chronic pain in general ranges from 30-70\%. ${ }^{39}$ These results suggest that future studies should try to incorporate genetic factors besides clinical and psychological factors, when trying to unravel the predictors for CPSP.

When trying to identify genetic predictors, several research methods are available. An important method is based on the use and analysis of single nucleotide polymorphisms (SNPs), which allows to study the link between genetic factors and clinical syndromes. ${ }^{40}$ In a SNP, a single base is substituted by another base in a specific locus at the chromosome. This substitution leads to different alleles, which can ultimately result in the decoding of a different protein (i.e. 'loss of function'), or of the same protein with other functional capacities (i.e. 'gain of function'). ${ }^{41}$ Several SNPs in close proximity to each other, often form a haplotype, which implies that they are inherited together. ${ }^{41} \mathrm{~A}$ major limitation of the SNP analysis is based on the fact that most clinical syndromes, especially pain syndromes, are complex and are very rarely caused by only one specific SNP, but rather by many SNPs which act in a concerted action. In order to unravel most potential SNPs correlated with CPSP a genome-wide association study (GWAS) can be performed. In this method an extremely large amount (millions) of SNPS spread over the entire genome, are studied simultaneously. ${ }^{42}$ The downside of performing a GWAS, is that it requires replication of data in multiple very large patient cohorts. ${ }^{43}$ Another way to acquire more insight into SNPs that could possibly be associated with the development CPSP, is by performing a systematic analysis of the available literature. SNPs that have been associated with CPSP in relatively small and heterogeneous patient cohorts are interesting to research in a larger homogeneous cohort. Finally, identifying SNPS associated with CPSP is only the first step towards a better understanding of the development of CPSP. In light of what we already know about clinical and psychological risk factors for the development of CPSP, it is even more important to explore if the addition of genetic polymorphisms to pre-existing prediction models of CPSP results in an increased accuracy of these models and thus improves the understanding of the complex pain syndrome known as CPSP.

\section{Aims and outline of this thesis}

The aim of this thesis is to improve our insight in the complex interaction of clinical, demographic, psychological and genetic factors and the development of CPSP after a wide variety of surgical procedures. In order to achieve this, the following research questions will be addressed:

- $\mathrm{RQ}$ 1. What is the prevalence of chronic postsurgical pain (CPSP) after relatively minor procedures in an outpatient setting? 
- RQ 2. Which demographic, clinical and psychological factors are associated with the development of CPSP after outpatient surgery?

- RQ 3. Is moderate to severe preoperative pain associated with an increase in hospital costs and postoperative health care utilization after outpatient surgery?

- RQ 4. Which genetic polymorphisms are associated with the prevalence and severity of CPSP?

- RQ 5. Does the addition of genetic risk factors to an existing clinical and demographic prediction model for CPSP after hysterectomy increase the accuracy of this model?

The first and second research questions are answered in chapter II en III respectively. Chapter II describes the results of a large prospective cohort study, which was performed amongst patients undergoing a wide variety of outpatient surgical procedures. These patients were followed during the first postoperative year. Chapter III describes the prevalence and predictive factors of CPSP and in patients after knee arthroscopy. This surgical procedure was chosen because it was one of the most frequently performed outpatient procedures in the Netherlands. ${ }^{1}$

$\mathrm{RQ} 3$ is addressed in chapter IV, where we describe whether preoperative pain, an important risk factor for CPSP, was associated with increased hospital costs, health care utilization and absenteeism from work and other activities during the first postoperative year after outpatient surgery.

RQ 4 and RQ 5, both dealing with genetics and CPSP, are addressed in chapter $V$ en VI. In chapter $V$, the available literature with regard to the genetic polymorphisms associated with CPSP is reviewed. In chapter VI genetic polymorphisms which are associated with CPSP after hysterectomy for benign indication were studied in homogeneous patient cohort. The addition of these genetic polymorphisms to a previously published predictive model for CPSP in the same patient population, is also described. Chapter VII is a general discussion of our findings and describes possible future research directions. The findings of this thesis are summarized in chapter VIII. 


\section{References}

1. Operaties in het ziekenhuis; soort opname, leeftijd en geslacht, 1995-2010. 05-02-2014. http://statline.cbs.nl/Statweb/publication/?DM=SLNL\&PA=80386NED\&D1=a\&D2=0\&D3=0\&D4=a\&D5=| \&HDR=T\&STB=G4\%2cG1\%2cG2\%2cG3\&VW=T.

2. Gramke HF, de Rijke JM, van Kleef M, et al. The prevalence of postoperative pain in a cross-sectional group of patients after day-case surgery in a university hospital. Clin J Pain 2007; 23(6): 543-8.

3. Stessel B, Fiddelers AA, Joosten EA, Hoofwijk DM, Gramke HF, Buhre WF. Prevalence and Predictors of Quality of Recovery at Home After Day Surgery. Medicine 2015; 94(39): e1553.

4. Gan TJ. Poorly controlled postoperative pain: prevalence, consequences, and prevention. J Pain Res 2017; 10: 2287-98.

5. Pavlin DJ, Chen C, Penaloza DA, Polissar NL, Buckley FP. Pain as a factor complicating recovery and discharge after ambulatory surgery. Anesth Analg 2002; 95(3): 627-34.

6. Inspectie voor de gezondheidszorg I. Basisset Medisch Specialistische Zorg Kwaliteitsindicatoren. 2017. https://http://www.igj.nl/documenten/indicatorensets/2016/08/08/basisset-medisch-specialistischezorg-2017.

7. Kalkman CJ, Visser K, Moen J, Bonsel GJ, Grobbee DE, Moons KG. Preoperative prediction of severe postoperative pain. Pain 2003; 105(3): 415-23.

8. Ballantyne JC, Carr DB, deFerranti S, et al. The comparative effects of postoperative analgesic therapies on pulmonary outcome: cumulative meta-analyses of randomized, controlled trials. Anesth Analg 1998; 86(3): 598-612.

9. Kehlet $\mathrm{H}$, Jensen TS, Woolf CJ. Persistent postsurgical pain: risk factors and prevention. Lancet 2006; 367(9522): 1618-25.

10. Macrae WA. Chronic pain after surgery. Br J Anaesth 2001; 87(1): 88-98.

11. Gerbershagen HJ, Aduckathil S, van Wijck AJ, Peelen LM, Kalkman CJ, Meissner W. Pain intensity on the first day after surgery: a prospective cohort study comparing 179 surgical procedures. Anesthesiology 2013; 118(4): 934-44.

12. Macrae WA. Chronic post-surgical pain: 10 years on. Br J Anaesth 2008; 101(1): 77-86.

13. Werner MU, Kongsgaard UE. I. Defining persistent post-surgical pain: is an update required? Br J Anaesth 2014; 113(1): 1-4.

14. Lavand'homme P. The progression from acute to chronic pain. Curr Opin Anaesthesiol 2011; 24(5): 54550.

15. Fletcher D, Stamer UM, Pogatzki-Zahn E, et al. Chronic postsurgical pain in Europe: An observational study. Eur J Anaesthesiol 2015; 32(10): 725-34.

16. Shipton EA. The transition from acute to chronic post surgical pain. Anaesth Intensive Care 2011; 39(5): 824-36.

17. Chapman CR, Vierck CJ. The Transition of Acute Postoperative Pain to Chronic Pain: An Integrative Overview of Research on Mechanisms. J Pain 2017; 18(4): 359 e1- e38.

18. Devor M. Sodium channels and mechanisms of neuropathic pain. J Pain 2006; 7(1 Suppl 1): S3-S12.

19. Melzack R, Wall PD. Pain mechanisms: a new theory. Science 1965; 150(3699): 971-9.

20. Berger JV, Knaepen L, Janssen SP, et al. Cellular and molecular insights into neuropathy-induced pain hypersensitivity for mechanism-based treatment approaches. Brain research reviews 2011; 67(1-2): 282310.

21. Guan Z, Hellman J, Schumacher M. Contemporary views on inflammatory pain mechanisms: TRPing over innate and microglial pathways. F1000Research 2016; 5.

22. Ji RR, Chamessian A, Zhang YQ. Pain regulation by non-neuronal cells and inflammation. Science 2016; 354(6312): 572-7.

23. Parsons B, Schaefer C, Mann R, et al. Economic and humanistic burden of post-trauma and post-surgical neuropathic pain among adults in the United States. J Pain Res 2013; 6: 459-69. 
24. Hollisaaz MT, Noorbala MH, Irani N, et al. Severity of chronic pain affects health care utilization after kidney transplantation. Transplant Proc 2007; 39(4): 1122-5.

25. Bayman EO, Brennan TJ. Incidence and severity of chronic pain at 3 and 6 months after thoracotomy: meta-analysis. J Pain 2014; 15(9): 887-97.

26. Jin J, Peng $L$, Chen $Q$, et al. Prevalence and risk factors for chronic pain following cesarean section: a prospective study. BMC anesthesiology 2016; 16(1): 99.

27. Gjeilo KH, Klepstad P, Wahba A, Lydersen S, Stenseth R. Chronic pain after cardiac surgery: a prospective study. Acta Anaesthesiol Scand 2010; 54(1): 70-8.

28. Peters ML, Sommer M, de Rijke JM, et al. Somatic and psychologic predictors of long-term unfavorable outcome after surgical intervention. Ann Surg 2007; 245(3): 487-94.

29. Niraj G, Rowbotham DJ. Persistent postoperative pain: where are we now? Br J Anaesth 2011; 107(1): 25-9.

30. Katz J, Seltzer Z. Transition from acute to chronic postsurgical pain: risk factors and protective factors. Expert Rev Neurother 2009; 9(5): 723-44.

31. Aasvang E, Kehlet H. Chronic postoperative pain: the case of inguinal herniorrhaphy. Br J Anaesth 2005; 95(1): 69-76.

32. Cerfolio RJ, Price TN, Bryant AS, Sale Bass C, Bartolucci AA. Intracostal sutures decrease the pain of thoracotomy. The Annals of thoracic surgery 2003; 76(2): 407-11; discussion 11-2.

33. Katz J, Poleshuck EL, Andrus CH, et al. Risk factors for acute pain and its persistence following breast cancer surgery. Pain 2005; 119(1-3): 16-25.

34. Turk DC, Okifuji A. Perception of traumatic onset, compensation status, and physical findings: impact on pain severity, emotional distress, and disability in chronic pain patients. J Behav Med 1996; 19(5): 43553.

35. Theunissen HM, Peters ML, Bruce J, Gramke HF, Marcus MA. Preoperative Anxiety and Catastrophizing: A Systematic Review and Meta-analysis of the Association With Chronic Postsurgical Pain. Clin J Pain 2012; 28(9): 819-41.

36. Theunissen $M$, Peters $M L$, Schouten EG, et al. Validation of the surgical fear questionnaire in adult patients waiting for elective surgery. PloS one 2014; 9(6): e100225.

37. Bruce J, Thornton AJ, Powell R, et al. Psychological, surgical, and sociodemographic predictors of pain outcomes after breast cancer surgery: a population-based cohort study. Pain 2014; 155(2): 232-43.

38. Theunissen M, Peters ML, Schepers J, et al. Recovery 3 and 12 months after hysterectomy: epidemiology and predictors of chronic pain, physical functioning, and global surgical recovery. Medicine 2016; 95(26): e3980.

39. Clarke H, Katz J, Flor H, Rietschel M, Diehl SR, Seltzer Z. Genetics of chronic post-surgical pain: a crucial step toward personal pain medicine. Can J Anaesth 2015; 62(3): 294-303.

40. Carlton VE, Ireland JS, Useche F, Faham M. Functional single nucleotide polymorphism-based association studies. Human genomics 2006; 2(6): 391-402.

41. Alberts B, Lewis J, Bray D. Molecular Biology of the Cell; 2000.

42. Kraft P, Cox DG. Study designs for genome-wide association studies. Advances in genetics 2008; 60: 465504.

43. Geschwind DH, Flint J. Genetics and genomics of psychiatric disease. Science 2015; 349(6255): $1489-94$. 



\section{Chapter}

\section{Prevalence and predictive factors of chronic postsurgical pain and poor global recovery one year after outpatient surgery}

Daisy M.N. Hoofwijk MD, Audrey A.A. Fiddelers PhD, Madelon L. Peters PhD, Björn Stessel MD, Alfons G.H. Kessels MD MSc, Elbert A. Joosten PhD, Hans-Fritz Gramke MD PhD, and Marco A.E. Marcus MD PhD. 


\section{Abstract}

\section{Objectives}

To prospectively describe the prevalence and predictive factors of chronic postsurgical pain (CPSP) and poor global recovery in a large outpatient population at a university hospital, one year after outpatient surgery.

\section{Methods}

A prospective cross-sectional cohort study was performed. During eighteen months, patients presenting for preoperative assessment were invited to participate. Outcome parameters were measured using questionnaires at three timepoints: one week preoperatively, four days postoperatively and one year postoperatively. A value of $>3$ on an 11-point numeric rating scale (NRS) was considered to indicate moderate to severe pain. A score of $\leq 80 \%$ on the Global Surgical Recovery Index (GSR) was defined as poor global recovery.

\section{Results}

A total of 908 patients were included. The prevalence of moderate to severe preoperative pain was $37.7 \%$, acute postsurgical pain $26.7 \%$, and CPSP $15.3 \%$. Risk factors for the development of CPSP were surgical specialty, preoperative pain, preoperative analgesic use, acute postoperative pain, lack of optimism and poor preoperative quality of life. The prevalence of poor global recovery was $22.3 \%$. Risk factors for poor global recovery were previous related surgery, preoperative pain, preoperative analgesic use, surgical fear, acute postoperative pain, poor preoperative and acute postoperative quality of life, and related surgery during the first postoperative year.

\section{Discussion}

Moderate to severe CPSP after outpatient surgery is common, and should not be underestimated. Patients at risk for developing CPSP can be identified during the preoperative phase. 


\section{Introduction}

Acute postsurgical pain after outpatient surgery is a common problem, and both the prevalence and risk factors for the development of acute postsurgical pain have been extensively studied. ${ }^{1-8}$ The development of chronic postsurgical pain (CPSP) after outpatient surgery has been less extensively studied, even though it is a burden for the patient and society.

CPSP has been defined as pain persisting longer than two months after surgery. ${ }^{9}$ However, in studies that investigate the problem of CPSP, follow-up times vary widely from several months to years. ${ }^{9}, 10$ The prevalence of moderate to severe CPSP varies from $10 \%$ to $50 \%$, whereas $2 \%$ to $10 \%$ of patients report severe CPSP. ${ }^{11}$ CPSP is associated with negative outcomes such as a reduced quality of life and increased health care utilization. ${ }^{12,13}$ Some specific types of surgery such as thoracotomy, lower limb amputation, joint arthroplasty, breast cancer surgery and inguinal hernia repair have been extensively studied and have been associated with severe CPSP. ${ }^{11}$

The studies that address CPSP have mostly been performed in inpatients. ${ }^{11,14,15}$ With the exception of a few studies examining specific outpatient procedures (e.g. inguinal hernia repair, breast cancer surgery), research about the presence of CPSP after outpatient surgery is scarce. The prevalence and risk factors of CPSP after outpatient surgery might be different from those of inpatient surgery for several reasons. First of all, the extent and impact of outpatient surgery can be different with regard to tissue damage and anesthetic techniques. Second, previous studies have shown that the treatment of acute postoperative pain might be less optimal for outpatients than for inpatients. ${ }^{16,17}$ This might lead to more peripheral and central sensitization and consequently more severe CPSP. Third, the psychological impact of outpatient surgery might be different (e,g. less anxiety and catastrophizing), because the disease which constitutes the indication for surgery may be less severe and because of the absence of hospital admission. In this context it is important and justified to collect specific information about the prevalence and the predictors of CPSP after outpatient procedures. First of all, identifying patients at risk for developing CPSP might lead to better tailored pharmacotherapeutic and psychotherapeutic strategies to decrease the prevalence of CPSP. Secondly, more knowledge about the prevalence of CPSP after outpatient surgery will enable informing patients more accurately about this serious complication.

Global recovery after surgery measures overall functional recovery. CPSP is associated with a negative impact on the cost-effectiveness of outpatient surgery and it may cause absenteeism from work and social activities. ${ }^{14}$ However, not all patients experiencing CPSP may be functionally impaired, some may consider themselves fully recovered and participate in regular work and social activities. In contrast, other may experience suboptimal or emotional recovery without experiencing CPSP. Not much 
research regarding global recovery has been performed, but predictors for the development of CPSP might not be the same as predictors for a poor global recovery. ${ }^{18}$

The aim of this study is to prospectively describe the prevalence and predictive factors of CPSP, as well as the prevalence and predictive factors of a poor global recovery, one year after outpatient surgery, in a large group of outpatients treated at a university hospital.

\section{Materials and methods}

\section{Patients}

This study was designed as a prospective longitudinal study. It was approved by the institutional Ethics Committee of the Maastricht University Medical Center+, and all patients gave informed consent to participate. Every patient who underwent elective outpatient surgery was eligible for inclusion, regardless of the nature of the procedure. Potential participants were excluded if they were younger than 18 years, were unable to express themselves, were visually impaired, or if their understanding of the Dutch language was insufficient.

\section{Instruments}

Information was collected by means of three successive questionnaires; a preoperative pain questionnaire (Q1), an acute postsurgical pain questionnaire (Q2) and a chronic postsurgical pain questionnaire (Q3). Q1 contained questions regarding demographic factors (e.g. age, gender, educational level, work status), average and present pain intensity, the influence of pain on daily activities, prescribed analgesic use, over-thecounter analgesic use, the effect of analgesic use on pain severity, and the number of visits to a medical doctor or other health care professionals. Furthermore, patients were specifically asked to only rate the pain they thought was related to the indication for the surgical procedure. All questions regarding pain were measured on an 11-point numeric rating scale (NRS; where $0=$ no pain, and $10=$ worst pain imaginable). Q1 also contained parts of four validated questionnaires. For two of these, shortened versions were used to keep the total number of questions within limits en thus avoiding patient refusal due to long questionnaires (i.e. diminishing patient burden). To measure catastrophic thinking, six of the thirteen original items of the Pain Catastrophizing Scale (PCS) were used. These were the two questions loading highest on each of the three subscales (i.e. items 5 and 12 for Helplessness, items 9 and 11 for Rumination, and items 6 and 13 for Magnification). The selection of these items was based on unpublished data from our previous research. ${ }^{18,}{ }^{19}$ The eight-item Surgical Fear Questionnaire (SFQ) was included to measure fear of potential consequences of 
surgery. Four items refer to fear of short-term consequences (e.g. pain, side effects) and four items refer to fear of long-term consequences (e.g. deterioration of health). ${ }^{20}$ Also, four of the originally ten questions of the Life Orientation Test Revised (LOT-R) were used to measure the personality trait optimism. ${ }^{21}$ The four filler questions were omitted and the four questions (two positively phrased and two negatively phrased) loading highest on, respectively the optimism and pessimism factor in a previous study by our group were selected. ${ }^{18}$ Finally, the EuroQol (EQ-5D) was used to measure five dimensions of quality of life (i.e. mobility, self-care, usual activity, pain/discomfort and anxiety/depression). ${ }^{22}$

Q2 contained questions regarding average and present pain intensity, the influence of pain on daily activities, prescribed and over-the-counter analgesic use, the effect of analgesic use on pain severity, visits to health care professionals, postoperative sideeffects and complications (e.g. nausea, vomiting, wound infections). We specifically asked the patients to only rate the pain they thought was related to the surgical procedure. Q2 also contained the EQ-5D and the 1-item Global Surgical Recovery index (GSR), which represents a single question about the extend to which patients considered themselves recovered from the surgery ("if 100\% recovery means your health is back to the same level as it was before the surgery, what percentage of recovery are you at now?"). ${ }^{18}$ The GSR has been found to correlate well with a more elaborate instrument measuring surgical recovery. ${ }^{23} \mathrm{Q} 3$ contained the same questions as Q2, as well as a question about the number of sick days during the past year.

\section{Procedure}

Between November 2008 and April 2010, patients planned for outpatient surgery and presenting at the outpatient clinic for preoperative assessment at the Maastricht University Medical Center +, were asked to participate in our study. The purpose and methods of the study were explained by the anesthesiologist or physician assistant performing the assessment. If consent was obtained, the patient received an envelope containing an information letter about the study, Q1, Q2, and two return envelopes. If no contraindications were present, the patient also received a standardized prescription for postoperative analgesics (i.e. acetaminophen $1000 \mathrm{mg}$ four times a day, with an upgrade to acetaminophen/tramadol 650/75 mg four times a day). The patient received verbal and written instructions to start with acetaminophen and to switch to acetaminophen/tramadol in case of insufficient analgesia.

Patients were instructed to complete Q1 one week before the surgical procedure. Patients who did not return Q1 were considered to be unwilling to participate, and no further attempts to contact them were made. Q2 had to be completed four days after the surgery. Patients who did return Q1 but did not return Q2, were reminded by regular mail or telephone. One year after the procedure all participants received Q3 by regular mail. All clinical information (e.g. ASA physical status, surgical procedure, type of 
anesthesia, duration of the procedure, duration of hospital stay) was acquired by systematic chart review.

\section{Statistical analysis}

All patients who returned both Q1 (because this questionnaire contained part of the predictor variables) and Q3 (because this contained the outcome parameters) were included in the analyses. Patient characteristics and mean pain scores were evaluated using descriptive statistics. For Q1 and Q3 average pain intensity during the last week was used, for Q2 the actual pain intensity. An NRS $>3$ was defined as a cut-off value to define moderate to severe pain and an NRS $>7$ was defined as severe pain. ${ }^{24,25}$ A GSR $\leq$ $80 \%$ was defined as poor global recovery. ${ }^{18}$

Before analyzing the database for possible predictors of CPSP and poor global recovery, missing data were imputed using multiple data imputation according to the method described by Van Buuren et al. ${ }^{26}$ In this method each missing value is replaced by a plausible value based on their predicted distribution. We imputed each of these missing values ten times, leading to ten different data sets. Results are based on the pooled outcome based on these ten data sets. Only predictor values were imputed, missing outcome variables were not. First, univariate logistic regression analyses were performed for each predictive variable. Only variables with a $p$-value $<0.1$ were included in the final multiple logistic regression model. Stepwise forward multiple logistic regression analyses were subsequently performed to determine which variables were predictive of CPSP and of poor global recovery. The model was constructed using four successive steps, based on the availability of the variables during the preoperative and postoperative phase. The first step contained the variables that are easiest to obtain, before patients visit the outpatient clinic (i.e. sex, age, and surgical specialty). For the first step a forced entry method was used. The second step contained variables that are easily obtained during the preoperative assessment (i.e. ASA-level, work status, education level, previous surgery, preoperative pain, and preoperative analgesic use). The third step contained variables that are more difficult to obtain, but can still be obtained in the preoperative phase. All psychological variables were added to the model in this step because these variables require patients to fill out multiple questionnaires (i.e. expected postoperative pain, surgical fear, pain catastrophizing, optimism, and preoperative quality of life). The fourth step contained all variables that can only be obtained during or after the surgery (i.e. anesthetic technique, duration of the surgery, duration of the hospital admission, acute postsurgical pain, poor global recovery on the fourth postoperative day, postoperative nausea, postoperative infection, additional surgery during the first postoperative year, and postoperative quality of life). A $p$-value of $<0.05$ was considered to be statistically significant. To estimate the models' ability to discriminate between patients with and without CPSP and with good versus poor global recovery, the area under the curve (AUC) was 
calculated. In addition, a correlation analysis between CPSP and GSR was performed. All analyses were performed using SPSS version 20.0 (SPSS Inc, Chicago, IL) and STATA version 11.2 (StataCorp, Texas, USA).

\section{Results}

During eighteen months, 2500 patients were invited to participate. Of these, 1396 patients (56\%) returned Q1. Of all patients that returned Q1, $1282(92 \%)$ returned Q2 and of these patients 906 patients (71\%) returned Q3. Of the 114 patients that returned Q1 but not Q2, 26 patients (23\%) did return Q3 and were also included. Figure 1 depicts the flowchart of inclusion. Of the 932 patients that were included, 24 patients were excluded because the inclusion had not been in accordance with study protocol; three patients were younger than 18 years at the time of the surgery, one patient was mentally impaired, and 20 patients had undergone a planned inpatient procedure. This resulted in data of 908 patients for the final analyses. Of these patients 908 returned Q1, 883 patients returned Q2, and 908 patients returned Q3.

Procedures performed per surgical specialty are shown in Table 1. Patient characteristics are shown in Table 2. Because the patient characteristics were calculated before missing values were imputed, the results are presented as absolute numbers and percentages, and do not always add up to a total of 908 patients. A small majority of patients were female (56.4\%) and the mean age was 53 years (range 18 to 88 years). The average duration of surgery was 49 minutes. Most patients $(n=716)$ received general anesthesia, a small number received regional anesthesia (e.g. spinal anesthesia, peripheral nerve blocks, intravenous regional anesthesia, and retrobulbar or subTenon's block) in addition to or instead of general anesthesia. Of all patients, 575 (63.3\%) were treated on an ambulatory basis, and 310 (34.1\%) in terms of a short-stay admission ( $<24$ hours). Twenty three patients $(2.6 \%)$ had to stay in hospital for $>24$ hours ( 15 patients stayed for 3 days, 6 patients for 4 days and 2 patients for 5 days) because of unforeseen complications. Of all patients, 67 patients (7.4\%) underwent another surgical procedure during the first year after the surgery. This second surgery was related to the original procedure in 42 patients (4.6\%). 


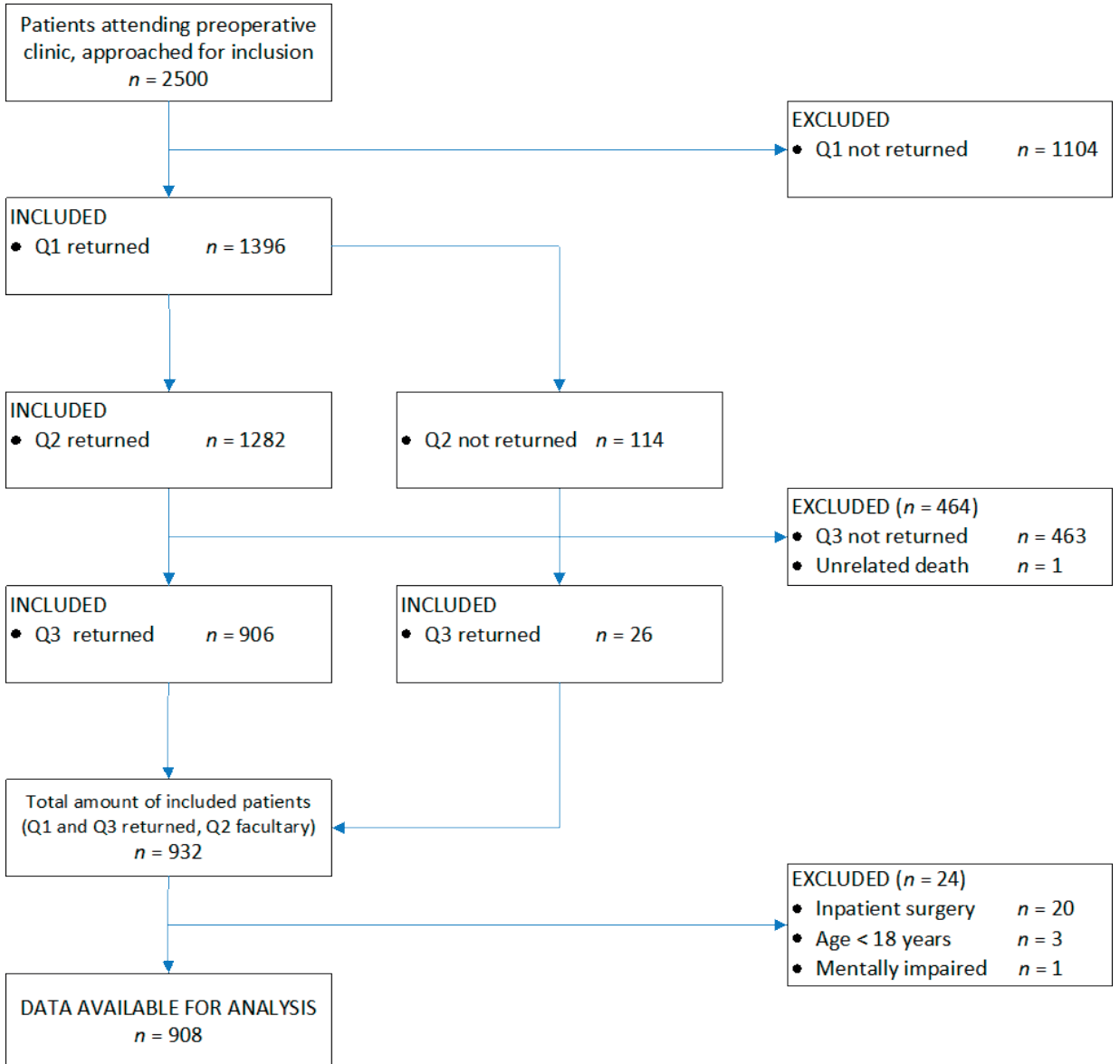

Figure 1: Flowchart depicting the inclusion and exclusion of the study population.

$\mathrm{Q} 1$ = questionnaire one week before the surgery; $\mathrm{Q} 2$ = questionnaire four days after the surgery; $\mathrm{Q} 3=$ questionnaire one year after the surgery

Table 1: Procedures performed by each surgical specialty

\begin{tabular}{ll}
\hline Ophthalmology $(n=70)$ & Urology $(n=55)$ \\
Cataract sugery / vitrectomy (49) & Brachytherapy prostate (26) \\
Strabismus surgery (11) & Scrotal surgery (11) \\
Dacrocystorhinostomy (5) & Uterine suspension surgery (11) \\
Other (5) & Other (7) \\
Gynaecology ( $n=70)$ & Plastic surgery ( $n=96)$ \\
Hysteroscopy (33) & Dupuytren fasciotomy (25) \\
Laparoscopic sterilisation / ovariectomy (20) & Breast reduction (11) \\
Surgical abortion (7) & Surgical correction of scar (9) \\
Vulva condylomata / Bartholin gland (5) & Tenolysis (9) \\
Diagnostic laparoscopy (3) & Replacement of breast implants (8) \\
Uterine suspension surgery (2) & Carpal tunnel release (7) \\
General surgery ( $n=271)$ & Skin biopsy / ganglion / lipoma (7) \\
Inguinal hernia repair (60) & Breast augmentation (6) \\
Lumpectomy (37) & Liposuction (3) \\
\hline
\end{tabular}




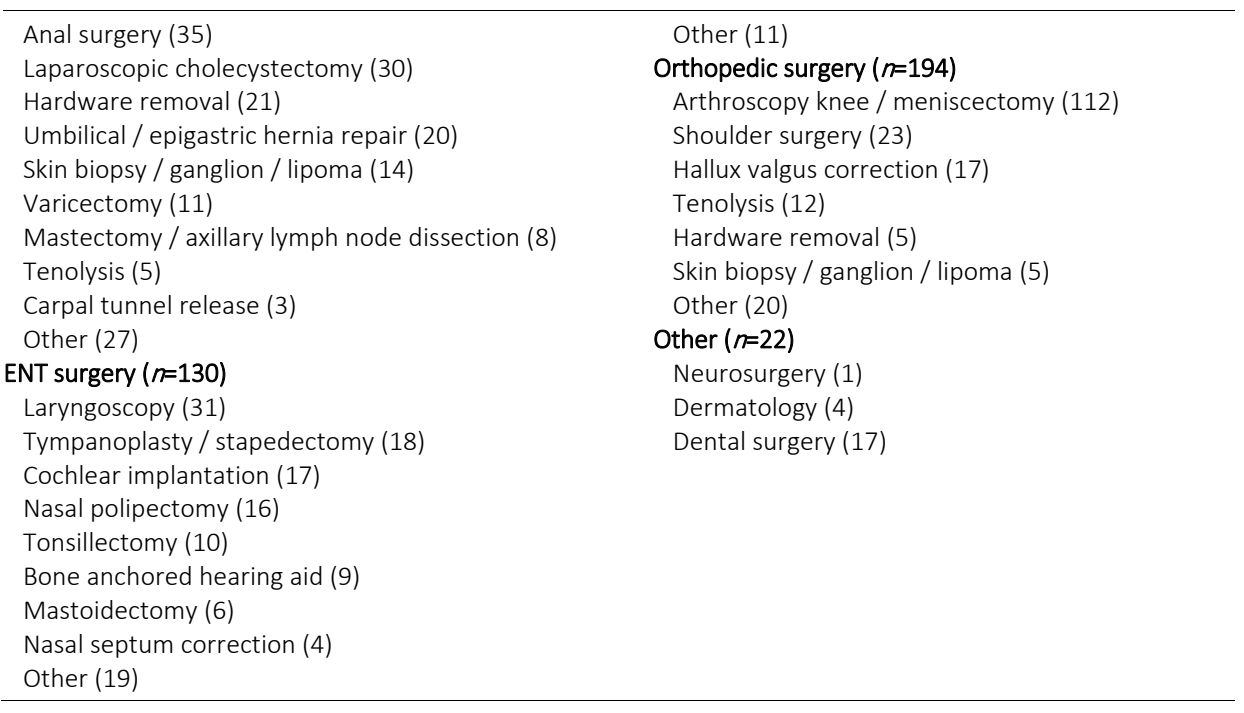

Table 2: Patient characteristics

\begin{tabular}{llll}
\hline & $N(\%)$ & $N(\%)$ \\
\hline $\begin{array}{l}\text { Age (years) } \\
<45\end{array}$ & $266(29.3)$ & Preoperative pain & NRS 0-3 \\
$45-59$ & $295(32.5)$ & NRS 4-10 & $553(62.3)$ \\
$>59$ & $347(38.2)$ & Preoperative analgesic use & $334(37.7)$ \\
Gender & & Yes & $220(24.5)$ \\
$\quad$ Male & $396(43.6)$ & No & $677(75.5)$ \\
$\quad$ Female & $512(56.4)$ & ASA physical status & \\
Educational background & I & II & $405(50.0)$ \\
$\quad$ Elementary school & $54(6.0)$ & III & $39(4.4)$ \\
Intermediate & $656(73.1)$ & Type of anesthesia & \\
$\quad$ Higher degree, university & $187(20.8)$ & General & $716(79.0)$ \\
Work situation & & Loco-regional & $143(15.8)$ \\
$\quad$ Working & $591(65.2)$ & General and loco-regional & $47(5.2)$ \\
Not working & $316(34.8)$ & & \\
\hline
\end{tabular}

NRS indicates numeric rating scale 


\section{Prevalence of pain}

A substantial number of patients $(n=334 ; 37.7 \%)$ experienced moderate to severe pain during the week before completing Q1. Four days after the surgery, 235 patients (26.7\%) experienced moderate to severe pain, and one year after surgery this was the case for 139 patients (15.3\%). A total of 29 patients (3.2\%) experienced severe CPSP.
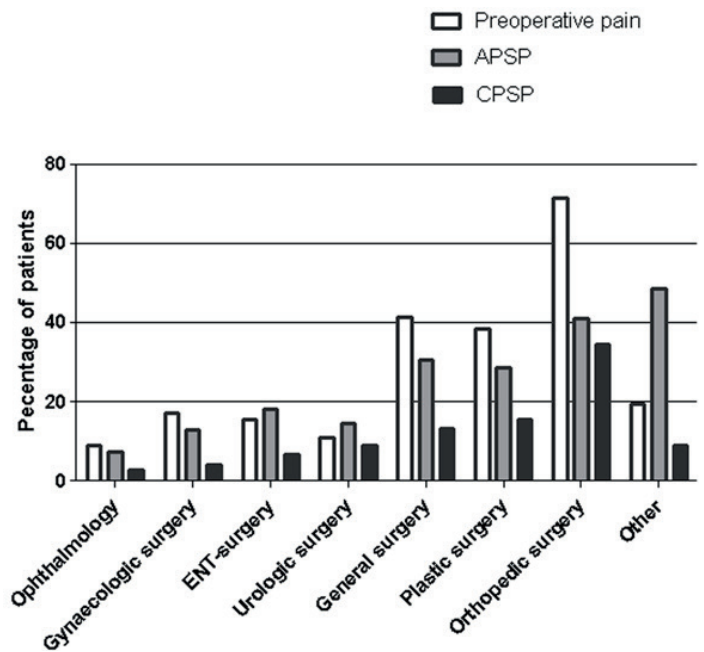

Figure 2: Prevalence of preoperative, acute postoperative and chronic postoperative pain according to type of surgery. Percentage of patients with a numeric rating scale $>3$. CPSP indicates chronic postsurgical pain.

Figure 2 shows the percentages of patients with moderate to severe preoperative, acute postoperative and chronic postsurgical pain for the different surgical specialties. During both the preoperative phase and the postoperative phase, orthopedic and general surgical procedures were most often associated with moderate to severe pain. Preoperative pain was rated as an NRS $>3$ bij $71.5 \%$ of orthopedic patients and by $41.4 \%$ of general surgical patients. Postoperatively, $41.2 \%$ and $30.5 \%$ of these patients scored an NRS $>3$. Orthopedic procedures were also most often associated with moderate to severe CPSP; $34.5 \%$ of the orthopedic patients scored and NRS > 3 one year after surgery. Other procedures that were relatively often associated with CPSP were general surgical and plastic surgical procedures. Ophthalmologic, urologic and gynecologic procedures resulted in the lowest pain scores at all three measuring points.

The prevalences of moderate to severe CPSP for the more extensively studied outpatient procedures was $13.1 \%$ for inguinal hernia repair, $10.0 \%$ for breast surgery, 9.6\% for general abdominal procedures and $10.0 \%$ for laparoscopic cholecystectomy. 


\section{Predictors of CPSP}

Table 3 shows the logistic regression analysis for CPSP and poor global recovery. No colinearity existed for any of the variables included. ASA-level, work status, educational level, previous surgery, expected postoperative pain, anesthetic technique, duration of the surgery, duration of hospital admission, postoperative nausea, postoperative infection, postoperative analgesic use, surgery during the first postoperative year, and postoperative quality of life, were all nonsignificant in the univariate logistic regression analyses and were therefore not included in this model. The most important predictors for CPSP after outpatient surgery were the surgical specialty, preoperative pain, preoperative analgesic use, surgical fear, and acute postsurgical pain. Of the patients with preoperative analgesic use and no preoperative pain (which is assumed to be adequately treated preoperative pain), the prevalence of CPSP was $8.0 \%$ as opposed to $32.0 \%$ for patients with moderate to severe preoperative pain (assumed to be inadequately treated preoperative pain). Higher optimism and higher preoperative quality of life were significant protective factors for the development of CPSP.

\section{Prevalence of Poor Global Recovery}

The average GSR index one year after outpatient surgery was $84.6 \%$ (SD 25.1\%). A total of 199 patients (22.3\%) scored a GSR of $\leq 80 \%$ and were thus defined as poor global recovery. Surgical specialties that were most often associated with poor global recovery were orthopedic surgery (i.e. GSR $\leq 80 \%$ in $39.2 \%$ of the patients), plastic surgery (24.7\%), ophthalmologic surgery $(22.9 \%)$, ear-nose-throat surgery $(21.8 \%)$, urologic surgery (20.0\%) and general surgery (17.8\%). Gynecologic surgery was least often associated with poor global recovery, only $5.9 \%$ of these patients scored a GSR $\leq 80 \%$.

\section{Predictors of Poor Global Recovery}

ASA-level, work status, expected postoperative pain, pain catastrophizing, anesthetic technique, duration of surgery, duration of hospital admission, postoperative nausea, postoperative infection, and postoperative analgesic use, were not statistically significant in the univariate logistic regression analysis en where not included in the final model. Significant predictors for poor global recovery were the surgical specialty (i.e. orthopedic surgery), preoperative pain, preoperative analgesic use, poor global recovery four days postoperative, surgical fear, related surgery during the year before the index surgery and follow-up surgery during the first postoperative year. Furthermore, higher preoperative and postoperative quality of life, as well as higher optimism, protected against a poor global recovery. 
Table 3: Results of the logistic regression analysis for CPSP (Numeric Rating Scale $>3$ ) and poor global recovery (Global Surgical Recovery Index < 80\%)

\begin{tabular}{|c|c|c|c|c|}
\hline Independent variables & $\begin{array}{l}\text { Chronic Postsurgical Pain } \\
\text { OR }(95 \% \mathrm{Cl})\end{array}$ & $A \cup C$ & $\begin{array}{l}\text { Poor Global Surgical } \\
\text { Recovery } \\
\text { OR }(95 \% \mathrm{Cl})\end{array}$ & $A \cup C$ \\
\hline Step 1 & & 0.73 & & 0.65 \\
\hline \multicolumn{5}{|l|}{ Age } \\
\hline Old vs. young & $0.99(0.98-1.00)$ & & $1.00(0.99-1.01)$ & \\
\hline \multicolumn{5}{|l|}{ Sex } \\
\hline Male vs. female & $0.82(0.55-1.21)$ & & $0.85(0.62-1.16)$ & \\
\hline \multicolumn{5}{|l|}{ Surgical specialty (vs. ophthalmology) } \\
\hline 1. Gynaecology & $1.21(0.48-3.09)$ & & $0.37(0.15-0.90)$ & \\
\hline 2. Ear-nose-throat surgery & $2.24(0.75-6.74)$ & & $0.83(0.42-1.61)$ & \\
\hline 3. Urology & $3.50(1.48-8.28)$ & & $0.84(0.55-1.27)$ & \\
\hline 4. General surgery & $4.97(2.37-10.42)$ & & $0.86(0.64-1.16)$ & \\
\hline 5. Plastic surgery & $5.89(2.72-12.75)$ & & $1.14(0.80-1.61)$ & \\
\hline 6. Orthopaedic surgery & $16.41(3.88-69.34)$ & & $2.67(1.96-3.62)$ & \\
\hline 7. Other & $2.65(0.35-20.16)$ & & $0.27(0.12-0.59)$ & \\
\hline Step 2 & & 0.80 & & 0.71 \\
\hline \multicolumn{5}{|l|}{ Educational level } \\
\hline Middle vs. low & & & $1.60(0.83-3.10)$ & \\
\hline High vs. low & & & $0.91(0.42-1.95)$ & \\
\hline \multicolumn{5}{|l|}{ Previous surgery } \\
\hline Not related & & & $1.40(0.86-2.30)$ & \\
\hline Related & & & $1.91(1.25-2.92)$ & \\
\hline \multicolumn{5}{|l|}{ Preoperative pain (NRS $>3$ ) } \\
\hline Yes vs. No & $4.22(2.54-7.02)$ & & $1.78(1.22-2.58)$ & \\
\hline \multicolumn{5}{|l|}{ Preoperative analgesic use } \\
\hline Yes vs. No & $2.16(1.40-3.34)$ & & $1.52(1.05-2.21)$ & \\
\hline Step 3 & & 0.85 & & 0.75 \\
\hline \multicolumn{5}{|l|}{ Surgical fear - long term } \\
\hline Per point (range 0-40 points) & $1.04(1.01-1.06)$ & & $1.03(1.01-1.05)$ & \\
\hline \multicolumn{5}{|l|}{ Optimism (LOT) } \\
\hline Per point (range 0-20 points) & $0.90(0.82-0.98)$ & & $0.89(0.83-0.95)$ & \\
\hline \multicolumn{5}{|l|}{ Preoperative QoL (EQ-5D) } \\
\hline Per point (range 0.59-1.00 points) & $0.09(0.04-0.22)$ & & $0.30(0.14-0.67)$ & \\
\hline Step 4 & & 0.85 & & 0.78 \\
\hline \multicolumn{5}{|l|}{ Acute postsurgical pain (NRS > 3 ) } \\
\hline Yes vs. No & $1.62(1.02-2.58)$ & & & \\
\hline \multicolumn{5}{|l|}{ Poor GSR on day 4 (GSR $\leq 80 \%)$} \\
\hline Yes vs. No & & & $2.41(1.44-4.04)$ & \\
\hline \multicolumn{5}{|l|}{ Surgery during first postoperative $y r$} \\
\hline Not related & & & $0.72(0.26-1.94)$ & \\
\hline Related & & & $2.64(1.27-5.49)$ & \\
\hline \multicolumn{5}{|c|}{ Acute postoperative Quality of Life (EQ-5D) } \\
\hline Per point (range 0.59-1.00 points) & & & $0.24(0.12-0.50)$ & \\
\hline
\end{tabular}

Step 1 used a forced entry method, while in the other steps only significant variables (univariate $p<0.1$ ) are entered. Odds ratio's (OR) with 95\% confidence interval (CI) and area under the curve (ACU) are presented per step. 


\section{Correlation Analysis}

Because the data for CPSP and GSR were not parametric, a Spearman $\rho$ was performed to determine the correlation between CPSP and GSR. The correlation coefficient was 0.656 ( $p<0.001)$. Furthermore, patients without CPSP have a median GSR index of $100 \%$ (interquartile range $90 \%$ to $100 \%$ ), and patients with CPSP have a median GSR index of $50 \%$ (interquartile range $25 \%$ - $75 \%$ ).

\section{Discussion}

In our study, $15.3 \%$ of all patients experienced moderate to severe CPSP after outpatient surgery, and $2.3 \%$ experienced severe CPSP. These findings fall in the range of the prevalences previously reported in several review papers. Kehlet et al reported a prevalence of $10 \%$ to $50 \%$ moderate to severe CPSP, and $2 \%$ to $10 \%$ severe CPSP. ${ }^{11}$ Another review article reports even higher incidences; $15 \%$ to $60 \%$ for moderate to severe CPSP and $4 \%$ to $10 \%$ for severe disabling CPSP. ${ }^{15}$ A recent publication researching only general surgical procedures, reported an incidence of $14.8 \%$ for moderate to severe CPSP. ${ }^{27}$ However, these and several other papers mostly describe surgical procedures that are usually not performed on an outpatient basis (e.g. limb

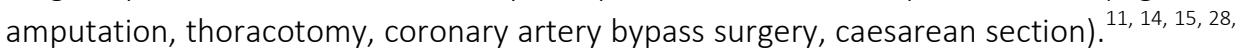
${ }^{29}$ So far, large-scale studies on CPSP after outpatient surgery are lacking; only relatively small studies focusing on specific outpatient procedures are available. There is reason to assume that both the prevalence and predictors of CPSP might be different in outpatients as compared with inpatients. Inpatient procedures are often associated with more extensive tissue damage, longer duration of hospital admission and a longer recovery period. In addition, the treatment of postoperative pain for outpatients might not be as optimal as for inpatients. ${ }^{16,17}$ Despite these dissimilarities the prevalence of CPSP found in the present study seems to be comparable with the ones reported for inpatient surgery.

One surgical procedure that has been extensively studied in the context of CPSP is inguinal hernia repair. The prevalence of CPSP for these procedures was $13.1 \%$ in our study, which is within the range reported by other studies (5\% to $35 \%$ ). ${ }^{11,14,28,30,31}$ One potentially important predictor for the development of CPSP after inguinal hernia repair is the surgical technique (e.g. mesh vs. non-mesh, open vs. laparoscopic). ${ }^{30,} 32$ Unfortunately, our study did not include and document data about the various surgical techniques.

The prevalence of CPSP after breast surgery has also been extensively studied. We found a CPSP prevalence of $10 \%$ after breast surgery, which contrasts to $20 \%$ to $50 \%$ reported in other major studies. ${ }^{11,14,28,33}$ Most studies have examined CPSP after surgery for breast cancer (i.e. mastectomies and lumpectomies). The procedures in our 
study, however, were lumpectomies $(n=37)$, a few mastectomies $(n=8)$, and the remaining were cosmetic surgical procedures $(n=25)$. A small retrospective study focusing uniquely cosmetic breast augmentation, found a CPSP prevalence of $9.5 \%{ }^{34}$ This might indicate that CPSP is more frequent after surgery for breast cancer than after cosmetic breast surgery. This might be explained by the fact that surgery for breast cancer is often associated with axillary surgery (i.e. nerve damage), chemotherapy and radiotherapy, all of which are risk factors for the development of CPSP. ${ }^{14,33}$

A third outpatient procedure that has previously been studied in the context of CPSP is cholecystectomy. In our population, CPSP occurred in $9.6 \%$ of the patients after general abdominal procedures and in $10 \%$ of patients after laparoscopic cholecystectomy. This in line with the $5 \%$ to $50 \%$ previously reported. ${ }^{14,35}$

In addition to reporting the prevalence of CPSP after outpatient procedures, another major aim of our study was to identify predictors of CPSP after outpatient surgery. Previous studies on risk factors for the development of CPSP have mostly dealt with inpatient procedures. ${ }^{11,14}$ As mentioned above, the factors identified for these procedures may not necessarily be the same as those for outpatient procedures. To identify the factors that were related to the presence of CPSP, we performed a stepwise logistic regression analysis. The first step in the logistic regression model contained easily obtainable variables, such as age, sex and surgical specialty. In our model these three variables were capable of a reasonably adequate prediction (AUC 0.73), which was mostly dependent on the surgical specialty. Orthopedic surgery was associated with the most severe CPSP. Orthopedic procedures are often associated with preoperative pain (which is often the reason to operate) and acute postoperative pain. These patients might therefore be prone to peripheral and central sensitization, which might cause the development of CPSP. Age and sex were not found to be significant predictors in our model. Previous studies found inconsistent evidence regarding age and sex as risk factors for CPSP, although various studies suggest that higher age may be associated with a decreased chance of developing CPSP. ${ }^{11,14,36}$

The second step of our model contained variables that can be easily obtained during preoperative assessment. This step significantly improved our model to an AUC of 0.80 . The presence of moderate to severe preoperative pain was shown to be the most important predictor of the entire model. Most studies addressing CPSP have also demonstrated this association. ${ }^{14,18,30,36}$ A possible reason for this association is the fact that peripheral and central sensitization may develop more easily in patients with long lasting pain. The presence of CPSP after orthopedic procedures might, however, also be a continuation of the preoperative pain, especially when pain was the indication for the surgery and the surgery was unsuccessful. Another important predictor for CPSP in our model was preoperative analgesic use, which has also been demonstrated before. ${ }^{37}$ It may, however, not be the use of analgesics in itself that is predictive of CPSP, but rather the efficacy of preoperative analgesic use. Our study demonstrated that patients with preoperative analgesics but without preoperative pain experienced significantly less 
CPSP than patients with inadequately treated preoperative pain. Treating preoperative pain with the correct and sufficient dose of analgesics might thus be a way of decreasing the prevalence and severity of CPSP. More research on this topic is needed.

The third step of our logistic regression analysis slightly improved our model by adding the psychological variables. Psychological risk factors for the development of CPSP after inpatient surgery have been extensively studied and especially anxiety and pain catastrophizing have been demonstrated to be associated with the risk of developing CPSP. ${ }^{10,14,18}$ In our study, all psychological variables included were associated with CPSP in the univariate analyses. In the multivariate analysis, long-term surgical fear, optimism, and quality of life remained in the model. Pain catastrophizing was no longer significant in the multivariate analyses. It might be speculated that patient undergoing minor outpatient procedures are less prone to catastrophize compared with patients undergoing more extensive inpatient procedures. Another explanation might lie in the use of a shortened version of the Pain Catastrophizing Scale, which might be less reliable. An alternative interpretation for the different findings amongst studies might be that anxiety, pain catastrophizing and optimism are to some extent correlated with each other. Letting them compete in a multivariate regression analysis might thus be artificial and lead to unstable results. In that case, another approach could be to identify broader psychological profiles, such as was done by Bruce et al. ${ }^{33}$ A composite variable consisting of high optimism, high positive affect, and low trait anxiety and depression, which they called 'psychological robustness' was associated with a decreased risk of developing CPSP after breast cancer surgery.

The fourth step added all perioperative and postoperative variables. The only statistically significant predictor for CPSP after outpatient surgery in this step was the presence of acute postoperative pain. Acute postoperative pain has been reported to be an important predictor for CPSP in other studies. ${ }^{11,14,18,32}$ In our population acute postoperative pain also predicted CPSP, but our model improved only slightly. Hence, acute postoperative pain seems less important than preoperative pain in predicting CPSP. It might be speculated that neuroplastic changes related to the development of CPSP, like peripheral and central sensitization, are more extensive and severe after long lasting preoperative pain than after relatively brief acute postoperative pain.

In addition to identifying predictors for CPSP, we also examined predictors of global perceived recovery. Some predictors for poor global recovery were identical to those for CPSP. This is not surprising, as pain is part of overall functional recovery and the two outcomes showed a substantial correlation. Nevertheless some additional predictors were identified: related surgery in the year before the surgical procedure, as well as preoperative an postoperative quality of life. Undergoing multiple operations within a relatively short timeframe and already being in a suboptimal condition as indicated by lower quality of life scores, may negatively impact the recovery process. Our model for predicting poor global recovery was fairly good (AUC 0.76), even though all four steps 
were necessary to achieve this predictability. However, more research on this subject is needed.

Our study included all outpatient procedures performed in our hospital, which has led to a heterogeneous study population. However, so far hardly any research on CPSP in a general outpatient population had been performed and the aim of this study was to examine whether CPSP is an important problem in this population as well. The heterogeneity was statistically corrected for by adding the surgical specialty to our regression model. Unfortunately, the groups of patients were too small to perform spate regression analyses for all the different kind of procedures performed. The results do demonstrate that some procedures are more often associated with CPSP than others (e.g. orthopedic surgery). However, when other possible predictors were added to the model, such as preoperative pain and analgesic use, the contribution of the surgical specialty became less prominent. This demonstrates that there may be common factors predicting outcome of various surgical procedures.

Our study has several limitations. The first limitation of our study might be that it is not possible to limitation it is not possible to determine whether CPSP was a new problem or whether it was a continuation of already existing preoperative pain. The proposed definition by Macrae states that this should be ruled out. ${ }^{9}$ Nevertheless, the aim of our study was to identify patients at risk for developing CPSP and preoperative pain seemed to be the single most important risk factor. For the individual patient is does not matter whether it is a new problem or not, they simply want to be able to prevent it or treat it. Future studies, however, should try to establish detailed assessment to determine whether the postsurgical pain is indeed newly developed pain. Another limitation is that patients were not asked to differentiate between pain at rest an pain with movement. Several previous studies have made this distinction and have shown that there can be a difference. ${ }^{33,}{ }^{35}$ We recommend that future studies do differentiate between pain at rest and pain with movement.

An important next step will be to investigate if and how it is possible to prevent CPSP, especially in patients at risk. As preoperative pain seems to be the single most important risk factor, one might hypothesize that a strategy of effectively treating this pain might reduce the incidence of CPSP. Whether it is possible to reverse the central sensitization that has already taken place, is a topic for future research. We feel that patients undergoing surgery should be informed that CPSP is an important problem, even after outpatient procedures and this information should be used as one of the determinants as to whether an elective surgical procedure should take place or not.

In conclusion, our study demonstrates that CPSP after outpatient surgery is an important problem, with $15.3 \%$ of all patients experiencing moderate to severe pain one year after the surgery. The preoperative phase proved to be extremely important in identifying patients at risk. By asking patients about preoperative pain and analgesic use, and determining the surgical specialty, it may be possible to reasonably accurately predict the chance of developing CPSP. This prediction might be somewhat improved by 
identifying patients with an unfavorable psychological profile, exemplified by a lack of optimism, high levels of surgical fear, and a low preoperative quality of life in this study. Although acute postoperative pain has previously been found to be an important risk factor, the addition of this factor only slightly improved our predictive model. 


\section{References}

1. Wu CL, Berenholtz SM, Pronovost PJ, Fleisher LA. Systematic review and analysis of postdischarge symptoms after outpatient surgery. Anesthesiology 2002; 96(4): 994-1003.

2. Rawal N. Postoperative pain treatment for ambulatory surgery. Best Pract Res Clin Anaesthesiol 2007; 21(1): 129-48.

3. McGrath B, Elgendy H, Chung F, Kamming D, Curti B, King S. Thirty percent of patients have moderate to severe pain $24 \mathrm{hr}$ after ambulatory surgery: a survey of 5,703 patients. Can J Anaesth 2004; 51(9): 88691.

4. Gramke HF, de Rijke JM, van Kleef M, et al. The prevalence of postoperative pain in a cross-sectional group of patients after day-case surgery in a university hospital. Clin J Pain 2007; 23(6): 543-8.

5. Brattwall M, Warren Stomberg M, Rawal N, Segerdahl M, Jakobsson J, Houltz E. Patients' assessment of 4-week recovery after ambulatory surgery. Acta Anaesthesiol Scand 2011; 55(1): 92-8.

6. Beauregard L, Pomp A, Choiniere M. Severity and impact of pain after day-surgery. Can J Anaesth 1998; 45(4): 304-11.

7. Gramke HF, de Rijke JM, van Kleef $M$, et al. Predictive factors of postoperative pain after day-case surgery. Clin J Pain 2009; 25(6): 455-60.

8. Ip HY, Abrishami A, Peng PW, Wong J, Chung F. Predictors of postoperative pain and analgesic consumption: a qualitative systematic review. Anesthesiology 2009; 111(3): 657-77.

9. Macrae WA. Chronic pain after surgery. Br J Anaesth 2001; 87(1): 88-98.

10. Theunissen HM, Peters ML, Bruce J, Gramke HF, Marcus MA. Preoperative Anxiety and Catastrophizing: A Systematic Review and Meta-analysis of the Association With Chronic Postsurgical Pain. Clin J Pain 2012; 28(9): 819-41.

11. Kehlet H, Jensen TS, Woolf CJ. Persistent postsurgical pain: risk factors and prevention. Lancet 2006; 367(9522): 1618-25.

12. Nickel R, Raspe HH. [Chronic pain: epidemiology and health care utilization]. Der Nervenarzt 2001; 72(12): 897-906.

13. Parsons B, Schaefer C, Mann R, et al. Economic and humanistic burden of post-trauma and post-surgical neuropathic pain among adults in the United States. J Pain Res 2013; 6: 459-69.

14. Macrae WA. Chronic post-surgical pain: 10 years on. Br J Anaesth 2008; 101(1): 77-86.

15. Lavand'homme P. The progression from acute to chronic pain. Curr Opin Anaesthesiol 2011; 24(5): 54550.

16. Watt-Watson J, Chung F, Chan VW, McGillion M. Pain management following discharge after ambulatory same-day surgery. J Nurs Manag 2004; 12(3): 153-61.

17. Solca M, Savoia G, Mattia C, et al. Pain control in day surgery: SIAARTI guidelines. Minerva Anestesiol 2004; 70(1-2): 5-24.

18. Peters ML, Sommer M, de Rijke JM, et al. Somatic and psychologic predictors of long-term unfavorable outcome after surgical intervention. Ann Surg 2007; 245(3): 487-94.

19. Sullivan MJ, Bishop SR. The Pain Catastrophizing Scale: Development and Validation. Psychological Assessment 1995; 7(4): 524-32.

20. Theunissen $M$, Peters ML, Schouten EG, et al. Validation of the surgical fear questionnaire in adult patients waiting for elective surgery. PloS one 2014; 9(6): e100225.

21. Scheier MF, Carver CS, Bridges MW. Distinguishing optimism from neuroticism (and trait anxiety, selfmastery, and self-esteem): a reevaluation of the Life Orientation Test. J Pers Soc Psychol 1994; 67(6): 1063-78.

22. van Agt HM, Essink-Bot ML, Krabbe PF, Bonsel GJ. Test-retest reliability of health state valuations collected with the EuroQol questionnaire. Soc Sci Med 1994; 39(11): 1537-44.

23. Kleinbeck SV. Self-reported at-home postoperative recovery. Res Nurs Health 2000; 23(6): 461-72. 
24. Gerbershagen HJ, Rothaug J, Kalkman CJ, Meissner W. Determination of moderate-to-severe postoperative pain on the numeric rating scale: a cut-off point analysis applying four different methods. Br J Anaesth 2011; 107(4): 619-26.

25. Dihle A, Helseth S, Paul SM, Miaskowski C. The exploration of the establishment of cutpoints to categorize the severity of acute postoperative pain. Clin J Pain 2006; 22(7): 617-24.

26. van Buuren S, Boshuizen HC, Knook DL. Multiple imputation of missing blood pressure covariates in survival analysis. Stat Med 1999; 18(6): 681-94.

27. Simanski CJ, Althaus A, Hoederath S, et al. Incidence of chronic postsurgical pain (CPSP) after general surgery. Pain Med 2014; 15(7): 1222-9.

28. Niraj G, Rowbotham DJ. Persistent postoperative pain: where are we now? Br J Anaesth 2011; 107(1): 25-9.

29. Reddi D, Curran N. Chronic pain after surgery: pathophysiology, risk factors and prevention. Postgraduate medical journal 2014; 90(1062): 222-7; quiz 6.

30. Aasvang EK, Gmaehle E, Hansen JB, et al. Predictive risk factors for persistent postherniotomy pain. Anesthesiology 2010; 112(4): 957-69.

31. Bay-Nielsen M, Perkins FM, Kehlet H, Danish Hernia D. Pain and functional impairment 1 year after inguinal herniorrhaphy: a nationwide questionnaire study. Ann Surg 2001; 233(1): 1-7.

32. Aasvang E, Kehlet H. Chronic postoperative pain: the case of inguinal herniorrhaphy. Br J Anaesth 2005; 95(1): 69-76.

33. Bruce J, Thornton AJ, Powell R, et al. Psychological, surgical, and sociodemographic predictors of pain outcomes after breast cancer surgery: a population-based cohort study. Pain 2014; 155(2): 232-43.

34. von Sperling ML, Hoimyr H, Finnerup K, Jensen TS, Finnerup NB. Persistent pain and sensory changes following cosmetic breast augmentation. Eur J Pain 2011; 15(3): 328-32.

35. Bisgaard T, Rosenberg J, Kehlet H. From acute to chronic pain after laparoscopic cholecystectomy: a prospective follow-up analysis. Scand J Gastroenterol 2005; 40(11): 1358-64.

36. Katz J, Seltzer Z. Transition from acute to chronic postsurgical pain: risk factors and protective factors. Expert Rev Neurother 2009; 9(5): 723-44.

37. Keller SM, Carp NZ, Levy MN, Rosen SM. Chronic post thoracotomy pain. J Cardiovasc Surg (Torino) 1994; 35(6 Suppl 1): 161-4. 



\section{Chapter}

\section{Prevalence and predictive factors of chronic}

postsurgical pain and global surgical recovery one year after outpatient knee arthroscopy; a prospective cohort study

Daisy M.N. Hoofwijk MD, Audrey A.A. Fiddelers PhD, Peter J. Emans MD PhD, Elbert A. Joosten, PhD, Hans-Fritz Gramke MD PhD, Marco A.E. Marcus MD PhD, Wolfgang F.F.A. Buhre MD PhD 


\section{Abstract}

\section{Introduction}

Outpatient knee arthroscopy is one of the most commonly performed surgical procedures. Previous research has demonstrated that chronic postsurgical pain (CPSP) after outpatient surgery is prevalent. Our objective was to determine the prevalence and predictive factors of CPSP and Global Surgical Recovery (GSR) one year after knee arthroscopy.

\section{Methods}

A prospective longitudinal cohort study was performed. Patients were included during an eighteen month period. Data were collected by using three questionnaires: at one week preoperatively, four days postoperatively and one year postoperatively. A value of $>3$ on an 11-point numeric rating scale (NRS) was defined as moderate to severe pain. A score of $\leq 80 \%$ on the Global Surgical Recovery Index was defined as poor GSR. Stepwise logistic regression analysis was performed to determine which variables were predictors for CPSP and poor GSR.

\section{Results}

The prevalence of moderate to severe preoperative pain in patients undergoing knee arthroscopy ( $n=104$ ) was $71.2 \%$, of acute postsurgical pain $37.5 \%$, and of CPSP $32.7 \%$. Risk factors for CPSP were the presence of preoperative pain and preoperative analgesic use, with odds ratios of $6.31(1.25-31.74)$ and 4.36 (1.58-12.07) respectively. The prevalence of poor GSR one year after outpatient knee arthrosocpy was $50.0 \%$. Poor GSR four days after the surgery was a risk factor with an odds ratio of 8.38 (0.92-76.58) and quality of life four days after surgery was a protective factor with and odds ratio of $0.10(0.02-0.64)$.

\section{Discussion}

Both CPSP and poor GSR are common one year after knee arthroscopy. Patients at risk for CPSP can be identified during the preoperative phase. Prediction of poor GSR one year after surgery is mainly related to early postoperative recovery. 


\section{Introduction}

Arthroscopic knee surgery is one of the most commonly performed surgical procedures, especially in the outpatient setting. Indications for performing this procedure include functional complaints, as well as acute or persisting pain. Recently, the effectiveness of arthroscopic knee surgery has been questioned for various indications and is now considered controversial. ${ }^{1}$ Several randomized studies have been performed to compare optimal conservative treatment or sham surgery with arthroscopic knee surgery for various diagnoses, but were not able to demonstrate a benefit of surgical intervention. ${ }^{2-5}$ Nevertheless, due to methodological constraints, correct interpretation of the results is difficult. First of all, no study has yet been performed to determine which variables, besides the presence of osteoarthritis, might predict whether a patient might benefit from surgical intervention or not. In this respect, variables like those related to socio-demographic, clinical and psychological predictors are of main interest. Second, most studies determine the effectiveness of arthroscopic knee surgery in terms of functional complaints. In this context, the question of both acute and chronic postsurgical pain (APSP and CPSP) should not be overlooked, since pain is most often the major indication for surgery, and at the same time pain is also considered to be an important complication of the surgical procedure. ${ }^{6,7}$ Both APSP and CPSP have been associated with several negative consequences for the patient's general health, the cost-effectiveness of the surgical procedure and for society in general. ${ }^{8-10}$ Another important outcome parameter is global surgical recovery (GSR), measuring the patient's satisfaction and overall success of the procedure. ${ }^{11}$ Patients who experience CPSP might consider themselves fully recovered and participate in regular work and social activities. In contrast, some patients without CPSP, might experience suboptimal overall recovery. Interestingly, previous studies have demonstrated that predictors of poor GSR might not be identical to predictors of CPSP. ${ }^{7,12}$

The aim of this study is to describe the prevalence and predictive factors of chronic postsurgical pain and poor global surgical recovery one year after outpatient knee arthroscopy in order to be able to identify the patients who are at risk.

\section{Methods}

\section{Subjects}

This study is a subgroup analysis of a previously published prospective longitudinal cohort study performed to collect information about the prevalence and predictive factors of APSP and CPSP, and GSR in patients undergoing outpatient surgery. ${ }^{7}$ Approval to perform this study was given by the Ethics Committee of the Maastricht University 
Medical Center+ (MUMC+), and all patients gave written informed consent to participate. The study included all adult patients undergoing outpatient surgery, regardless of the nature of the procedure. Patients were excluded if they were younger than 18 years, were unable to express themselves, were visually impaired, or if their understanding of the Dutch language was insufficient. In this present article, only patients who underwent arthroscopic knee surgery are described.

\section{Instruments}

Information was collected by using three questionnaire packages; a preoperative pain questionnaire package (Q1), an acute postsurgical pain questionnaire package (Q2) and a chronic postsurgical pain questionnaire package (Q3). Q1 contained questions about preoperative pain intensity, analgesic use, health care utilization, quality of life, as well as questions about demographic and psychological candidate predictors. Q2 and Q3 contained questions about pain intensity, analgesic use, health care utilization, quality of life, global recovery, and postoperative side effects. ${ }^{7,12}$ All questions regarding pain were measured on an 11-point numeric rating scale (NRS; where $0=$ no pain, and $10=$ worst pain imaginable). Furthermore, patients were specifically asked if they thought the pain they were experiencing was related to the surgery. Global surgical recovery (GSR) was measured by using the Global Surgical Recovery index, which represents a single question about the extend to which patients considered themselves recovered from the surgery ("if $100 \%$ recovery means your health is back to the same level as it was before the surgery, what percentage of recovery are you at now ?"). ${ }^{12}$ In order to measure the patient's psychological status four validated questionnaires were used (i.e. Pain Catastrophizing Scale, Surgical Fear Questionnaire, Life Orientation Test Revised and the EuroQol). ${ }^{13-16} \mathrm{~A}$ detailed description of the questions asked and the questionnaires used, can be found elsewhere. ${ }^{7}$

\section{Study design}

Between November 2008 and April 2010, patients scheduled for elective outpatient surgery at the MUMC+ were asked to participate if they were planned for outpatient surgery. In case of consent, patients received an envelope with the first two questionnaires and a standardized prescription for postoperative analgesics (i.e. acetaminophen $1000 \mathrm{mg}$ four times a day, with an upgrade to acetaminophen/tramadol $650 / 75 \mathrm{mg}$ four times a day in the case of insufficient analgesia). Patients were asked to complete Q1 one week before the surgery and Q2 four days after the surgery. Patients who returned $\mathrm{Q} 1$, but not $\mathrm{Q} 2$, were reminded to complete $\mathrm{Q} 2$ by telephone or mail. Patients received Q3 one year after the surgery. All clinical information (e.g. ASA physical status, type of anesthesia, duration of the procedure, duration of hospital stay) was acquired by systematic chart review. The severity of osteoarthritis was graded 
during the arthroscopic procedure, according to the Outerbridge Classification. ${ }^{17}$ This classification consists of a 5-point scale, where 0 is no osteoarthritis, 1 is softening and swelling of articular cartilage, 2 is fragmentation and fissuring of articular cartilage affecting < 0.5 inches, 3 is fragmentation and fissuring of articular cartilage affecting > 0.5 inches, and 4 is cartilage erosion to the bone.

\section{Outcome measures}

The main outcome variables in this study were chronic postsurgical pain and global surgical recovery. In accordance with previous studies, NRS > 3 was used to define moderate to severe pain. ${ }^{7,18,19}$ Global surgical recovery was defined as poor when the GSR-index was $\leq 80 \%{ }^{7,12}$

\section{Statistical analysis}

Only patients who returned all three questionnaires were included in the analyses. In order to evaluate patient characteristics and mean pain scores, descriptive statistics were used. For Q1 and Q3 the average pain intensity during the last week was used, for Q2 the actual pain intensity.

We recently identified variables that were relevant predictors for the development of CPSP or poor GSR. ${ }^{7}$ The following variables were included in the analyses: age, gender, preoperative pain, preoperative analgesic use, expected postoperative pain, surgical fear, preoperative quality of life, early postoperative quality of life, acute postoperative pain, acute postoperative global recovery, comorbid osteoarthritis, and additional surgery during the first postoperative year. Missing data were imputed using multiple data imputation according to the Van Buuren method. ${ }^{20}$ Only missing predictors variables were imputed, missing outcome variables were not.

Predictor variables were initially tested in a univariate logistic regression analysis. If the $p$-value was $<0.1$, the variable was also included in the multiple logistic regression model. Stepwise forward four-step multiple logistic regression analysis was performed. The steps were based on the availability of the variables during the perioperative phase. The first step contained variables that were easiest to obtain; before the patient visits the outpatient clinic (i.e. gender and age). A forced entry method was used for the first step. The second step contained variables that can easily be obtained during the preoperative visit (i.e. preoperative pain and preoperative analgesic use). The third step contained variables that can be obtained preoperatively, but require more detailed questionnaires (i.e. expected postoperative pain, surgical fear and preoperative quality of life). The fourth step consisted of variables that can only be obtained during or after the surgery (i.e. comorbid osteoarthritis according to the Outerbridge Classification, acute postoperative pain, acute postoperative global recovery, additional surgery during the first postoperative year, acute postoperative quality of life. A $p$-value of 
$<0.05$ was considered to be statistically significant. The area under the curve (AUC) was calculated to determine the models' ability to discriminate. All analyses were performed by SPSS version 20.0 (SPSS Inc, Chicago, IL) and STATA version 11.2 (StataCorp, College Station, TX)

\section{Results}

Of the 908 patients that underwent outpatient surgery and were included into our database, 104 patients fulfilled the inclusion criteria of the current substudy. For the flowchart of the original 908 patients we would like to refer to our previous publication. ${ }^{7}$ Patient characteristics of the 104 patients are given in Table 1 . Because the patient characteristics were calculated before multiple imputation, the results do not always add up to a total of 104 patients. The mean age of the patients was 53.1 years (standard deviation 13.6 years) and there were 54 male and 50 female patients. Forty patients (38.5\%) used analgesics during the week before the surgery, and 62 patients (59.6\%) did not. A total of 8 patients (7.7\%) underwent another surgical procedure during the first postoperative year (i.e. 6 patients underwent total knee arthroplasty, 1 patient underwent a different arthroscopic procedure of the same knee, and 1 patient underwent anterior cruciate ligament reconstruction).

Table 1: Baseline patient characteristics.

\begin{tabular}{llll}
\hline & $\mathrm{N}(\%) /$ & $\mathrm{N}(\%)$ \\
\hline Age (years) & & Type of surgery \\
$<45$ years & $28(26.9)$ & Lavage and debridement & $21(20.2)$ \\
$\geq 45$ years & $76(73.1)$ & Partial meniscectomy & $81(77.9)$ \\
Gender & & Microfracture chondral defect & $2(1.9)$ \\
Male & $54(51.9)$ & Indication for surgery & \\
Female & $50(48.1)$ & Pain & $98(94.2)$ \\
Educational background & & Symptoms of locking & $15(14.4)$ \\
Elementary school & $8(7.7)$ & Instability & $7(6.7)$ \\
Intermediate & $80(76.9)$ & Effusion & $5(4.8)$ \\
Higher degree, university & $16(15.4)$ & Outerbridge Classification & \\
Work situation & & 0 and 1 (no osteoarthritis) & $30(28.8)$ \\
Paid work & $53(51.0)$ & 2 and 3 (mild osteoarthritis) & $39(37.5)$ \\
Unpaid work & $23(22.1)$ & 4 (severe osteoarthritis) & $16(15.4)$ \\
Not working & $28(26.9)$ & Type of anesthesia & \\
ASA physical status & & General & $50(48.1)$ \\
I & $52(50.0)$ & Spinal & $44(42.3)$ \\
II & $46(44.2)$ & Combination & \\
III & $3(2.9)$ & & \\
\hline
\end{tabular}

NRS = Numeric Rating Scale 


\section{Prevalences of acute and chronic postsurgical pain and poor global recovery}

The prevalences of preoperative pain, APSP and CPSP, as well as the GSR of patients undergoing knee arthroscopy are demonstrated in Table 2. Seventy-four patients (71.2\%) experienced moderate to severe pain during the week before surgery. In 72 patients (69.2\%) pain interfered with usual activities. Thirty-nine patients (37.5\%) experienced moderate to severe APSP on the fourth postoperative day. Pain interference had not decreased. Thirty-four patients (32.7\%) experienced moderate to severe CPSP one year after the procedure, and in 32 patients (30.8\%) pain interfered with daily activities. One year after the knee arthroscopy, 17 patients (16.4\%) reported more pain than preoperatively, 13 patients (12.5\%) reported the same amount of pain, and 74 patients $(71.1 \%)$ reported less pain than preoperatively. Table 3 demonstrates the percentage of patients with APSP and CPSP for patients with and without moderate to severe preoperative pain.

Ninety-two patients (88.5\%) scored a GSR $\leq 80 \%$ four days after the surgery, 9 patients (8.7\%) scored GSR $>80 \%$ and thus showed good recovery, and of 3 patients data were missing. One year after surgery, 52 patients (50.0\%) presented a GSR $\leq 80 \%$, 48 patients (46.2\%) a GSR $>80 \%$, and the data of 4 patients were missing.

Table 2: Preoperative, acute postoperative and chronic postoperative pain intensity and global surgical recovery index.

\begin{tabular}{llll}
\hline & Preoperative & $\begin{array}{l}4 \text { days } \\
\text { postoperative } \\
\mathrm{N}(\%)\end{array}$ & $\begin{array}{l}1 \text { year } \\
\text { postoperative } \\
\mathrm{N}(\%)\end{array}$ \\
\hline $\begin{array}{l}\text { Global Surgical Recovery Index (\%) } \\
\text { Median (IQR) }\end{array}$ & & $50(40-70)$ & $80(60-99)$ \\
Mean pain intensity1 (NRS 0-10) & & & \\
Median (IQR) & $5(3-7)$ & $4(2-6)$ & $2(0-5)$ \\
NRS 0-3 & $29(27.9)$ & $46(44.2)$ & $70(67.3)$ \\
NRS 4-10 & $74(71.2)$ & $58(55.8)$ & $34(32.7)$ \\
Information missing & $1(1.0)$ & - & - \\
Present pain intensity (NRS 0-10) & & & $1(0-3)$ \\
Median (IQR) & $5(2-6)$ & $3(1-4)$ & $80(76.9)$ \\
NRS 0-3 & $41(39.4)$ & $65(62.5)$ & $24(23.1)$ \\
NRS 4-10 & $62(59.6)$ & $39(37.5)$ & - \\
Information missing & $1(1.0)$ & - & $2(0-4)$ \\
Pain interference with usual activities (NRS 0-10) & & & $72(69.2)$ \\
Median (IQR) & $5(3-7)$ & $5.5(3-7)$ & $32(30.8)$ \\
NRS 0-3 & $30(28.8)$ & $32(30.8)$ & - \\
NRS 4-10 & $72(69.2)$ & $72(69.2)$ & - \\
Information missing & $2(1.9)$ & - & \\
\hline
\end{tabular}

$\mathrm{IQR}=$ Interquartile Range. NRS = Numeric Rating Scale. 
Table 3: Number and percentages of patients with acute postsurgical pain (APSP) and chronic postsurgical pain (CPSP) for patients with and without moderate to severe preoperative pain.

\begin{tabular}{lll}
\hline Preoperative NRS & APSP & CPSP (\%) \\
& $n(\%)$ & $n(\%)$ \\
\hline NRS 0-3 $(n=29)$ & $5(17.2)$ & $2(6.9)$ \\
NRS 4-10 $(n=75)$ & $34(45.6)$ & $32(42.7)$ \\
\hline
\end{tabular}

NRS = numeric rating scale

\section{Predictors of CPSP and poor global recovery}

Based on a logistic regression analysis for CPSP and poor GSR no colinearity for any of the variables was observed (Table 4). Surgical fear, acute postoperative GSR, the presence of ostheoarthritis and additional surgery in the year after the index procedure, were not statistically significant predictors for CPSP in the univariate analysis and were thus not added to the final model. Variables predictive of CPSP in the multiple regression model were the presence of preoperative pain and the preoperative use of analgesics (AUC of 0.78). The only significant predictors for poor GSR one year after surgery, were poor GSR four days after the surgery and poor quality of life four days after the procedure (AUC of $0.70)$. Surgical fear, the presence of comorbid osteoarthritis and additional surgery in the year after the index procedure were no predictors for poor GSR.

The severity of osteoarthritis according to the Outerbridge Classification versus the GSR and pain intensity one year after knee arthroscopy is shown in figure 1 and figure 2.

Table 4: Results of the linear regression analysis for chronic postsurgical pain (CPSP) and global surgical recovery (GSR).

\begin{tabular}{|c|c|c|c|c|}
\hline Independent variable & $\begin{array}{l}\text { CPSP } \\
\text { OR }(95 \% \mathrm{CI})\end{array}$ & $A \cup C$ & $\begin{array}{l}\text { Poor GSR } \\
1 \text { yr after surgery } \\
\text { OR }(95 \% \mathrm{Cl})\end{array}$ & $A \cup C$ \\
\hline Step 1 & & 0.57 & & 0.52 \\
\hline \multicolumn{5}{|l|}{ Age } \\
\hline$\geq 45$ yrs vs. $<45$ yrs & $0.80(0.32-2.02)$ & & $1.00(0.42-2.43)$ & \\
\hline \multicolumn{5}{|l|}{ Gender } \\
\hline Male vs. female & $0.68(0.29-1.56)$ & & $0.84(0.38-1.85)$ & \\
\hline Step 2 & & 0.78 & & - \\
\hline \multicolumn{5}{|l|}{ Preoperative pain (NRS $>3$ ) } \\
\hline Yes vs. no & $6.31(1.25-31.74)$ & & & \\
\hline \multicolumn{5}{|l|}{ Preoperative analgesic use } \\
\hline Yes vs. no & $4.36(1.58-12.07)$ & & & \\
\hline Step 3 & & - & & - \\
\hline Step 4 & & - & & 0.70 \\
\hline \multicolumn{5}{|l|}{ GSR 4 days after surgery } \\
\hline$>80 \%$ vs. $\leq 80 \%$ & & & $8.38(0.92-76.58)$ & \\
\hline \multicolumn{5}{|l|}{ QOL 4 days after surgery } \\
\hline Per point (range -0.59-1.00 points) & & & $0.10(0.02-0.64)$ & \\
\hline
\end{tabular}

Step 1 used a forced entry method, while in other steps only significant variables (univariate $p<0.1$ ) are entered. Area under the curve $(A \cup C)$ are presented per step. NRS = numeric rating scale. $Q O L=$ quality of life. 


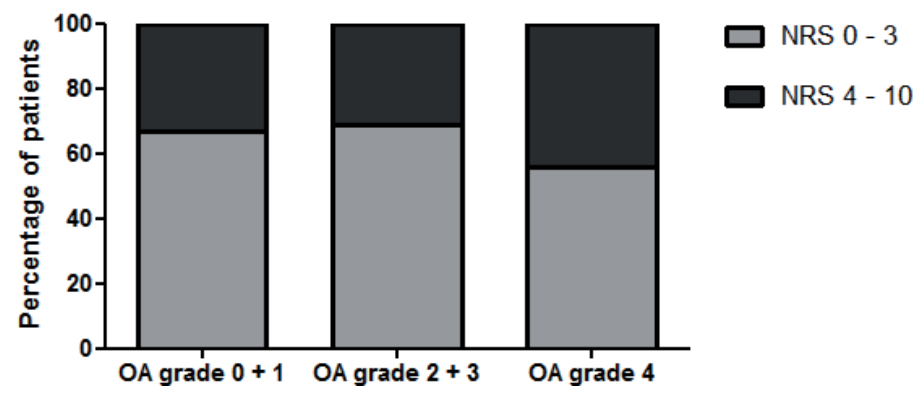

Figure 1: Presence of chronic postsurgical pain (CPSP) versus the severity of osteoarthritis according to the Outerbridge Classification. NRS = numeric rating scale, OA = osteoarthritis

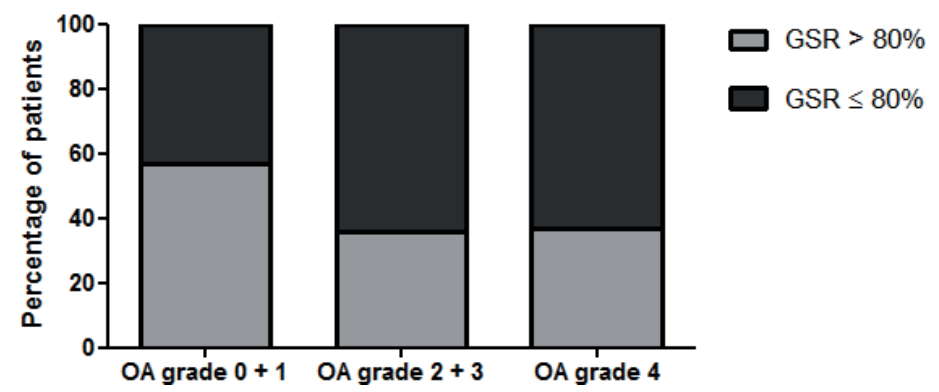

Figure 2: Presence of poor global surgical recovery (GSR) versus the severity of osteoarthritis according to the Outerbridge Classification. OA = osteoarthritis

\section{Discussion}

In this study we report a prevalence of preoperative pain in patients that underwent arthroscopic knee surgery of $71.2 \%$, a prevalence of acute postsurgical pain of $37.5 \%$ and a prevalence of chronic postsurgical pain one year after surgery of $30.8 \%$. During the last decade, the prevalence of CPSP after several inpatient procedures was reported to vary between $10 \%$ and $60 \% .{ }^{21-23}$ A more recent large cross-sectional study reported a prevalence of $18.3 \%$ of moderate to severe CPSP after various surgical procedures. ${ }^{24}$ With respect to knee arthroscopy a CPSP prevalence of $30 \%$ (NRS $\geq 1$ ) was reported one year after surgery. ${ }^{25}$ The prevalence of CPSP after knee arthroscopy as reported in our study is higher than reported by Rosseland and colleagues, but it should be noted that in their study only patients with moderate acute postoperative pain were included, whereas all patients with preoperative pain or severe acute postoperative pain were excluded. For that reason, the results of Rosseland and colleagues and our results regarding the prevalence of CPSP one year after knee arthroscopy are difficult to compare. Closely related, but not identical, data on the prevalence of CPSP in patients 
after joint arthroplasty have been published. ${ }^{26-28}$ It should be taken into account that arthroscopic knee surgery is a relatively minor outpatient procedure, which causes less tissue damage and no or even shorter hospital admission compared to joint arthroplasty. Nevertheless, the prevalence of CPSP after joint arthroplasty, varying between $37.5 \%$ six months after total hip replacement, 50.5\% three months after total knee replacement, and $37.5 \%$ one year after total shoulder replacement, does not significantly differ from our data in patients after arthroscopic knee surgery. ${ }^{26-28}$ This might indicate that, in case of orthopedic surgery, the extent of tissue damage and the duration of hospital admission are no reliable predictors for CPSP compared to other variables.

We previously reported a CPSP prevalence of $15.3 \%$ after various surgical outpatient procedures. ${ }^{7}$ This suggests that CPSP occurs more often after knee arthroscopy (32.7\%) compared to other outpatient procedures. This increased prevalence might be explained by a high prevalence of preoperative pain in these patients populations (71.2\% for arthroscopic knee surgery versus $37.7 \%$ for various outpatient procedures). Preoperative pain has proven to be one of the most important risk factors for the development of CPSP. . $^{2} 12,21,23,28,29$

Another aim of this study was to analyze risk factors of CPSP after outpatient knee arthroscopy. Age and gender were included into the first step of the logistic regression model. Neither age nor gender proved to be a significant predictor of CPSP in our model. Large recent studies on CPSP in a general population, as well as smaller studies performed in a specific orthopedic population, could not identify gender as a predictable risk factor for CPSP. ${ }^{7,12,24,26,28}$ In contrast, conflicting results have been reported for age. Some studies have not been able to find an effect, while other large studies observed an increasing chance of developing CPSP with decreasing age. $7,12,24,26,28$ This finding is not supported by the results of the present study in patients undergoing knee arthroscopy.

Significant predictors in the second step of our model were the presence of preoperative pain and preoperative analgesic use. The addition of these variables into the regression model resulted in an AUC of 0.78. As mentioned before, preoperative pain has been proven to be an important predictor of CPSP. ${ }^{7,12,21,23,28,29}$ This is most probably related to the fact that preoperative pain causes both peripheral and central sensitization, which in turn can cause CPSP to develop more easily. Preoperative analgesic use was also an important predictor of CPSP. We previously demonstrated that patients with preoperative analgesic use and preoperative pain were more likely to develop CPSP than patients with adequately treated preoperative pain. ${ }^{7}$ We therefore hypothesize that it is not analgesic use in itself that is the risk factor for CPSP, but rather the inefficacy of the analgesic use.

Psychological variables were added to the regression model in the third step. As these variables were not statistically significant in predicting CPSP, they were discarded from the model. Psychological traits have been extensively studied and previous 
research has demonstrated that these variables (e.g. surgical fear, pain catastrophizing, depression, pessimism) are important risk factors for the development of CPSP. ${ }^{7,12,27,29,30}$ We hypothesize that these variables were not significant in this study because knee arthroscopy is a relatively minor procedure without a large emotional aspect, as opposed to other surgical procedures (e.g. oncologic surgery).

All further perioperative and postoperative variables were added to the regression model in the fourth step, but none of these variables were statistically significant. Acute postoperative pain has been proven to be an important predictor for the development of CPSP after varying procedures. ${ }^{7,12,21,29}$ One small scale study did not report a predictive effect of acute postoperative pain on CPSP in patients that underwent total hip arthroplasty. ${ }^{26}$ The conclusion of this study is at least debatable as the absence of a significant effect might be related to the small amount of patients included. In our previous large-scale study, acute postoperative pain was shown to be a significant predictor of CPSP, although it only slightly improved the prediction model. ${ }^{7}$ We speculated that long-lasting preoperative pain is a more relevant predictor, because long-lasting preoperative pain might cause more extensive neuroplastic changes compared to brief acute postoperative pain. Additional related surgery in the first postoperative year was also added to the regression model in the fourth step, but was not a predictor. One could argue that this was not statistically significant because of the small number of patients that actually underwent a second procedure $(n=8)$. However, the prevalence of CPSP in these patients was $37.5 \%$ as opposed to $32.3 \%$ in the patients that did not undergo another related surgical procedure.

Another important variable that was added to the regression model was the severity of osteoarthritis according to the Outerbridge Classification. The degree of osteoarthritis was not a significant predictor, although we did observe a trend towards an increasing prevalence of CPSP in patients with concomitant grade 4 osteoarthritis. In this respect two relevant studies have been published. ${ }^{3,4}$ The first study described patients with a meniscal tear and concomitant osteoarthritis, and could not demonstrate any benefit of arthroscopy versus optimized physical therapy. ${ }^{4}$ The second study studied patients with a meniscal tear without osteoarthritis, and could also not find any benefit of arthroscopy versus sham surgery. ${ }^{3}$ The authors hypothesize that a meniscal tear could be an early sign of knee osteoarthritis rather than a separate clinical problem. It should, however, be noted that in this study patients with a traumatic meniscal rupture were excluded, while specifically this subpopulation might benefit from arthroscopy surgery. Unfortunately, in both studies no subgroup analysis was done in order to determine which patients might benefit from surgery and which patients might not. Here we did not observe a predictive effect of osteoarthritis on CPSP, but the present study was not powered for this secondary outcome. On the other hand, we observed a trend of an increased risk for the development of CPSP in patients with grade 4 osteoarthritis (figure 1a). In order to better answer the question whether the degree of osteoarthritis is a predictor of CPSP after knee arthroscopy, an adequate 
trial comparing knee arthroscopy with nonsurgical intervention in patients with a meniscal tear, with and without osteoarthritis and patients with and without a traumatic cause should be performed. Then subgroup analyses would have to be performed to determine which patients might benefit from surgical intervention.

The second outcome parameter of this study was global surgical recovery (GSR). This parameter was chosen because it measures overall recovery and previous studies have demonstrated that the risk factors for developing CPSP might not be identical to those for poor GSR. ${ }^{7,12}$ A substantial amount of patients in this study (i.e. 50.0\%) experienced poor GSR one year after knee arthroscopy. This percentage of $50.0 \%$ is relatively high as compared to those reported in other studies. The study of Peters and colleagues reported a prevalence of $34.4 \%$ six months after various inpatient procedures. ${ }^{12}$ Recently, we reported a prevalence of $22.3 \%$ one year after various outpatient procedures, and $39.2 \%$ in the subset of patients undergoing ambulatory orthopedic surgery. ${ }^{7}$ Clearly, the prevalence of poor GSR after outpatient knee arthroscopy is amongst the highest of all procedures, including other orthopedic procedures. It would be interesting to know in which domain knee arthroscopy patients experience suboptimal recovery (e.g. functional, emotional, daily activities, social activities). We were able to define two significant risk factors for poor GSR one year after knee arthroscopy. First, poor GSR four days after surgery and second poor quality of life four days after surgery. Unfortunately, this implies that patients at risk for a long-term poor GSR after knee arthroscopy can only be identified after surgery. Hence the model cannot be used to predict preoperatively which patients might benefit from the surgery. Nevertheless, it would be of major interest to study whether modifications in postoperative therapy in patients with poor GSR four days after surgery, can change the one-year outcome.

The present study has a number of limitations. The most important limitation is that we were not able to determine if the CPSP was a newly developed postoperative problem, or rather a continuation of pre-existing preoperative pain. This might result in an overestimation of the incidence of CPSP after arthroscopic knee surgery. Table 3 shows that the prevalence of CPSP in patients without preoperative pain was $6.9 \%$. In these patients it is fairly certain that the newly acquired pain is CPSP and thus a complication of the procedure. Furthermore, we have demonstrated that $16.4 \%$ of the patients reports worse pain after one year than before the procedure. In patients with moderate to severe preoperative pain, the prevalence of CPSP was $42.7 \%$. One could argue that most of these patients have benefitted of the arthroscopic procedure, because fewer patients are in moderate to severe pain after one year. However, since the natural cause of the pain without arthroscopic knee surgery is unknown, our study is not able to either support or reject that theory either. Future studies should therefore focus on trying to establish whether CPSP is indeed newly developed pain. The second limitation of the present study is the fact that the performed arthroscopies were quite heterogeneous (i.e. lavage and debridement, partial meniscectomy, microfracture 
chondral defect). Variable procedures might result in small but significant changes in predicting CPSP and poor GSR, and this can lead to instabilities in the logistic regression model. However, we accepted this variability, because it reflects clinical reality much better than focusing on a specific group of arthroscopic treatments (e.g. meniscal tear). A third limitation of the present study was the fact that certain parameters were acquired by retrospective systematic chart review (i.e. performed arthroscopic procedure, indication for the surgery). This was necessary because the present study is based on a subgroup analysis of a previously performed study. ${ }^{7}$ For this reason we are not able to clarify the discrepancy between the prevalence of pain as the indication for the procedure (94.2\%) and preoperative pain as measured by our questionnaire (71.2\%). A possible explanation might be that orthopedic surgeons are more likely to look at a composite endpoint (e.g. consisting of pain, annoyance, bad feeling, functional complaints, etc.) instead of standardized pain questionnaires. A second possible explanation for the discrepancy could be the natural course of the pain. Patients may experience serious pain when visiting the orthopedic outpatient clinic, but the pain could have diminished by the time the surgery is performed, usually several weeks later. This would require a re-evaluation of the pain complaints immediately before performing the surgery.

In conclusion, our study demonstrates that a substantial amount of patients experienced CPSP after outpatient knee arthroscopy. Patients with preoperative pain and patients with preoperative analgesic use were at risk for experiencing CPSP. The prevalence of poor GSR one year after outpatient knee arthroscopy was 50\%. A poor GSR four days after surgery and poor quality of life four days after surgery were strong predictors for poor GSR one year after surgery. Even though increasing severity of osteoarthritis according to the Outerbridge Classification was not a significant predictor for either CPSP or poor GSR one year after surgery, we did demonstrate a trend towards a bad outcome. 


\section{References}

1. Krych AJ, Carey JL, Marx RG, et al. Does arthroscopic knee surgery work? Arthroscopy. May 2014;30(5):544-545.

2. Moseley JB, O'Malley K, Petersen NJ, et al. A controlled trial of arthroscopic surgery for osteoarthritis of the knee. N Engl J Med. Jul 11 2002;347(2):81-88.

3. Sihvonen R, Paavola M, Malmivaara A, et al. Arthroscopic partial meniscectomy versus sham surgery for a degenerative meniscal tear. N Engl J Med. Dec 26 2013;369(26):2515-2524.

4. Katz JN, Brophy RH, Chaisson CE, et al. Surgery versus physical therapy for a meniscal tear and osteoarthritis. N Engl J Med. May 2 2013;368(18):1675-1684.

5. Kirkley A, Birmingham TB, Litchfield RB, et al. A randomized trial of arthroscopic surgery for osteoarthritis of the knee. N Engl J Med. Sep 11 2008;359(11):1097-1107.

6. Reigstad O, Grimsgaard C. Complications in knee arthroscopy. Knee Surg Sports Traumatol Arthrosc. May 2006;14(5):473-477.

7. Hoofwijk DM, Fiddelers AA, Peters ML, et al. Prevalence and Predictive Factors of Chronic Postsurgical Pain and Poor Global Recovery One Year after Outpatient Surgery. Clin J Pain. Jan 62015.

8. Parsons B, Schaefer C, Mann R, et al. Economic and humanistic burden of post-trauma and post-surgical neuropathic pain among adults in the United States. J Pain Res. 2013;6:459-469.

9. Joshi GP, Ogunnaike BO. Consequences of inadequate postoperative pain relief and chronic persistent postoperative pain. Anesthesiol Clin North America. Mar 2005;23(1):21-36.

10. Pavlin DJ, Chen C, Penaloza DA, Polissar NL, Buckley FP. Pain as a factor complicating recovery and discharge after ambulatory surgery. Anesth Analg. Sep 2002;95(3):627-634.

11. Kleinbeck SV. Self-reported at-home postoperative recovery. Res Nurs Health. Dec 2000;23(6):461-472.

12. Peters ML, Sommer M, de Rijke JM, et al. Somatic and psychologic predictors of long-term unfavorable outcome after surgical intervention. Ann Surg. Mar 2007;245(3):487-494.

13. Osman A, Barrios FX, Kopper BA, Hauptmann W, Jones J, O'Neill E. Factor structure, reliability, and validity of the Pain Catastrophizing Scale. J Behav Med. Dec 1997;20(6):589-605.

14. Theunissen M, Peters ML, Schouten EG, et al. Validation of the surgical fear questionnaire in adult patients waiting for elective surgery. PloS one. 2014;9(6):e100225.

15. Scheier MF, Carver CS, Bridges MW. Distinguishing optimism from neuroticism (and trait anxiety, selfmastery, and self-esteem): a reevaluation of the Life Orientation Test. I Pers Soc Psychol. Dec 1994;67(6):1063-1078.

16. van Agt HM, Essink-Bot ML, Krabbe PF, Bonsel GJ. Test-retest reliability of health state valuations collected with the EuroQol questionnaire. Soc Sci Med. Dec 1994;39(11):1537-1544.

17. Outerbridge RE. The etiology of chondromalacia patellae. J Bone Joint Surg Br. Nov 1961;43-B:752-757.

18. Gerbershagen HJ, Rothaug J, Kalkman CJ, Meissner W. Determination of moderate-to-severe postoperative pain on the numeric rating scale: a cut-off point analysis applying four different methods. Br J Anaesth. Oct 2011;107(4):619-626.

19. Dihle A, Helseth S, Paul SM, Miaskowski C. The exploration of the establishment of cutpoints to categorize the severity of acute postoperative pain. Clin J Pain. Sep 2006;22(7):617-624.

20. van Buuren S, Boshuizen HC, Knook DL. Multiple imputation of missing blood pressure covariates in survival analysis. Stat Med. Mar 30 1999;18(6):681-694.

21. Kehlet $\mathrm{H}$, Jensen TS, Woolf CJ. Persistent postsurgical pain: risk factors and prevention. Lancet. May 13 2006;367(9522):1618-1625.

22. Lavand'homme P. The progression from acute to chronic pain. Curr Opin Anaesthesiol. Oct 2011;24(5):545-550.

23. Katz J, Seltzer Z. Transition from acute to chronic postsurgical pain: risk factors and protective factors. Expert Rev Neurother. May 2009;9(5):723-744.

24. Johansen A, Romundstad L, Nielsen CS, Schirmer H, Stubhaug A. Persistent postsurgical pain in a general population: prevalence and predictors in the Tromso study. Pain. Jul 2012;153(7):1390-1396. 
25. Rosseland LA, Solheim N, Stubhaug A. Pain and disability 1 year after knee arthroscopic procedures. Acta Anaesthesiol Scand. Mar 2008;52(3):332-337.

26. Clarke H, Kay J, Mitsakakis N, Katz J. Acute pain after total hip arthroplasty does not predict the development of chronic postsurgical pain 6 months later. J Anesth. Aug 2010;24(4):537-543.

27. Masselin-Dubois A, Attal N, Fletcher D, et al. Are psychological predictors of chronic postsurgical pain dependent on the surgical model? A comparison of total knee arthroplasty and breast surgery for cancer. J Pain. Aug 2013;14(8):854-864.

28. Bjornholdt KT, Brandsborg B, Soballe K, Nikolajsen L. Persistent pain is common 1-2 years after shoulder replacement. Acta orthopaedica. Feb 2015;86(1):71-77.

29. Macrae WA. Chronic post-surgical pain: 10 years on. Br J Anaesth. Jul 2008;101(1):77-86.

30. Theunissen HM, Peters ML, Bruce J, Gramke HF, Marcus MA. Preoperative Anxiety and Catastrophizing: A Systematic Review and Meta-analysis of the Association With Chronic Postsurgical Pain. Clin J Pain. 2012;28(9):819-841. 



\section{Chapter}

\section{Severity of preoperative pain is associated with the amount of postoperative hospital- based health care costs during the first year after outpatient surgery}

Daisy M.N. Hoofwijk MD, Audrey A.A. Fiddelers PhD, Björn Stessel MD PhD, Hans-Fritz Gramke MD PhD, Carmen D. Dirksen PhD, Sander M.J. van Kuijk PhD, Elbert A. Joosten PhD, Wolfgang F. Buhre MD PhD 


\section{Abstract}

\section{Objectives}

The aim of the study is to determine whether there is an association between the severity of preoperative pain and the amount of postoperative hospital-based health care costs during the first year after outpatient surgery. Identifying patients at risk for high postoperative health care costs during the preoperative phase might create possibilities for developing cost-effective interventions.

\section{Methods}

A prospective longitudinal cohort study was performed. The amount of postoperative hospital-based health care costs was gathered from our data management system. Patient characteristics and pain scores were measured by three questionnaires ( 1 week preoperatively, 4 days postoperatively and 1 year postoperatively). Multiple linear regression analysis was used to test the association between preoperative pain severity and postoperative hospital-based health care costs.

\section{Results}

1353 patients were included. The average amount of postoperative hospital-based health care costs was $€ 1.520$ ( $\mathrm{Cl} € 1.406-€ 1.633)$. Increasing preoperative pain severity was associated with higher postoperative hospital-based health care costs $(p<0,001)$, especially after orthopaedic and urologic surgery. Patients with moderate to severe preoperative pain (Numeric Rating Scale (NRS) > 3) visited their general practitioner, medical specialist or other health care professional significantly more often during the first postoperative year. These patients were also more often absent from paid work.

\section{Discussion}

Preoperative pain severity is positively associated with postoperative hospital-based health care costs during the first year after outpatient surgery. Future studies have to be performed to determine whether preoperative interventions are able to reduce postoperative health care expenditures in patients with preoperative pain. 


\section{Introduction}

Pain in general is an important health problem in the developed world, which is associated with increased consumption of health care resources, absenteeism from paid work and a substantial economic burden. ${ }^{1-5}$ Health care costs associated with chronic pain after surgery have been less extensively studied, even though previous studies have demonstrated that $10-60 \%$ of all surgical patients suffer from chronic postsurgical pain (CPSP). ${ }^{6-8}$ A small study reports that patients with severe neuropathic CPSP or post-trauma pain visit physicians more frequently and use more prescribed medication than patients with less severe neuropathic CPSP or post-trauma pain. ${ }^{9}$ Another small study reports that CPSP after kidney transplantation is associated with increased health care utilization. ${ }^{10}$ Even though these studies report an association between CPSP and health care utilization, they do not actually demonstrate an association between CPSP and increased health care costs.

We do know that preoperative pain is very strongly associated with the severity of CPSP. ${ }^{11-14}$ The general prevalence of preoperative pain is approximately $40 \%$, but varies widely between surgical specialties; from $9 \%$ for ophthalmologic surgery to $71 \%$ for orthopaedic surgery. ${ }^{11}$ A causal relationship between the severity of preoperative pain and CPSP, through neuroplastic changes, is likely. ${ }^{6}$ It is therefore thought that treating preoperative pain might lead to a decrease in the prevalence and severity of CPSP, and might thus lead to decreased postoperative health care utilization. There is some evidence suggesting that postoperative health care consumption could be reduced by preoperative neuroscience education in patients with preoperative pain. ${ }^{15}$ However, no literature is available that demonstrates a clear relationship between the severity of preoperative pain and postoperative health care consumption.

The primary aim of this study is to determine the amount of hospital-based health care costs during the first postoperative year after outpatient surgery and to determine whether there is an association between the severity of preoperative pain and the postoperative hospital-based health care costs. This study will be the first to explore this association. The first secondary aim is a subgroup analysis of this association between the severity of preoperative pain and the postoperative hospital-based health care costs per surgical specialty. Another secondary aim is the determination of other health care costs (e.g. primary care costs, paramedical costs) and indirect costs based on the loss of productivity for both patients with and without preoperative pain. 


\section{Materials and methods}

\section{Subjects}

This study was designed as a prospective longitudinal cohort study. It was approved by the Ethics Committee of the Maastricht University Medical Center+ (MUMC+), and all patients gave informed consent. All adult patients who were planned for elective outpatient surgery were eligible to participate, except patients with visual impairment, inability to express themselves or insufficient understanding of the Dutch language were excluded.

\section{Outcome measures}

The primary outcome measure was the amount of hospital-based health care costs during the first year after outpatient surgery and the relationship between the costs and the severity of preoperative pain. The first secondary outcome measure was the relationship between the costs and the severity of preoperative pain per surgical specialty. The other secondary outcome measures were the number of visits to a general practitioner, medical specialist, emergency department or other health care professional and the costs associated with these visits, as well as the number of absent days from paid work, school and regular activities, and the indirect costs associated by absenteeism from paid work (i.e. loss of productivity).

\section{Study design}

A large prospective cohort study was performed. The aim of the study was to identify the prevalence and risk factors of preoperative, acute postoperative and chronic postoperative pain after outpatient surgery, as well as the costs associated with these procedures and the severity of pain. The data regarding prevalence and risk factors for acute and chronic postsurgical pain have been published in another paper. ${ }^{11}$

An elaborate description of the study design is presented elsewhere. ${ }^{11}$ Patients planned for outpatient surgery were asked to participate when presenting at the outpatient clinic for preoperative assessment at the Maastricht University Medical Center+. Participating patients received three questionnaires. The first questionnaire (Q1) was to be completed one week before the surgery, the second questionnaire (Q2) on the fourth postoperative day and the third questionnaire (Q3) one year after the surgery. If no contraindications were present, patients received a standardized prescription for postoperative analgesics (acetaminophen $1000 \mathrm{mg} 4$ times a day, with an upgrade to acetaminophen/tramadol 650/75 mg 4 times a day).

Q1 was the baseline questionnaire and contained questions about demographic characteristics (e.g. age, gender, educational level, and work status), average and 
present pain intensity, the influence of pain on daily activities, analgesic use and the effect of analgesic use on pain severity, the number of visits to a healthcare professional during the past twelve months (i.e. general practitioner, medical specialist, emergency department, or other medical professional), as well as the number of days of absenteeism from work, school or other regular activities during the past twelve months. Q1 also contained parts of four validated questionnaires to evaluate psychological characteristics (i.e. Pain Catastrophizing Scale, Life Optimism Trait, Surgical Fear Questionnaire and EuroQol-5D). ${ }^{16-19}$

Q2 and Q3 were two similar questionnaires with questions about pain intensity, the influence of pain on daily activities, and analgesic use. Patients were asked about the number of postoperative visits to a medical professional because of pain, as well as the number of absent days from work, school or other regular activities since the surgical procedure. These questionnaires also contained the EuroQol-5D and the Global Surgical Recovery Index. ${ }^{19,20}$

Data about hospital-based health care costs (i.e. the primary outcome) were collected from the patient data management system of the MUMC+. We collected data about the health care use for each patient and calculated the associated costs, by using the standardized prices charged by the hospital in 2009. Data collection started the moment the patient left the hospital after the index surgery, until exactly one year after the surgery. The index surgery and the day admission were not included, because these were assumed to be the same for all patients. All hospital-based costs were included (e.g. visits to outpatient clinics, admissions, imaging techniques, etc.), with the exception of laboratory tests and histologic examinations.

Data about the number of visits to health care professionals and associated costs, as well as indirect costs (i.e. the secondary outcomes) were collected via the study questionnaires. Patients reported the number of visits with questions about pain to a general practitioner, medical specialist in any hospital, emergency department of any hospital, or other medical professional (e.g. physical therapists, chiropractor, etc.) since the surgery. Patients also reported the number of days they were absent from regular work activities, school or other social activities. Costs for these secondary outcomes were calculated by using Dutch standardized prices for $2009 .{ }^{21}$

\section{Statistical analysis}

Patients who returned Q1 were included in the analyses of the primary outcome parameter. For the secondary outcome parameters, only patients who returned both Q1 and Q3 were included, because these outcomes were reported by the patients in Q3. In accordance with previous publications, an NRS $>3$ was defined as moderate to severe pain. ${ }^{11,22,23}$ Patient characteristics were evaluated by using descriptive statistics. Differences in patient characteristics between patients with and without preoperative pain were evaluated by using the Mann-Whitney $U$ test and the Kruskal-Wallis test for 
continuous variables that were not normally distributed, and the Chi-square test for categorical variables.

Before performing the analyses for the primary and secondary outcomes, missing data were imputed using multiple imputation. The number of imputations was set to 5, and we used predictive mean matching to draw values. This method ensures no values beyond the range of observed values can be imputed, and is robust to non-normal distribution of variables. The results were pooled using Rubin's Rules if possible, with the exception of $p$-values. These are reported as a range because no universally valid method exists to pool $p$-values after multiple imputation. If no range is reported, all $p$ values were the same in the five datasets.

In order to analyse the association between the severity of preoperative pain and postoperative hospital-based health care costs, a multiple linear regression analysis was performed. Gender, age and severity of preoperative pain were included in the multiple linear regression model. Other preoperative variables were one by one included into the model. If, after inclusion of a variable, the regression coefficient of preoperative pain changed more than $10 \%$, the variable was considered to be a confounder and remained in the model. Preoperative variables that we tested were ASA physical status, educational level, work status, and psychological variables (i.e. optimism, pain catastrophizing and surgical fear).

For the first secondary outcome, subgroup multiple lineair regression analyses were performed for each surgical specialty. For the other secondary outcomes descriptive statistics were used. In order to differentiate between patients with and without moderate to severe preoperative pain, the Mann-Whitney $U$ test and the Kruskal-Wallis test for nonparametric data were used.

A $p$-value of $<0.05$ was considered to be statistically significant. All analyses were performed using SPSS version 24.0 (SPSS Inc, Chicage IL).

\section{Results}

\section{Flowchart and patient characteristics}

Between November 2008 and April 2010, 2500 patients were invited to participate. The flowchart of included and excluded patients is demonstrated elsewhere. ${ }^{11}$ Of all invited patients 1396 patients (56\%) returned Q1. Of these patients, 1353 patients did not meet any exclusion criteria and for these patients postoperative hospital based health care costs were calculated. These patients were used for the analyses of the primary outcome. A total of 912 patients $(67,4 \%)$ returned Q3 and were used for the analysis of the secondary outcomes.

The patient characteristics are shown in Table 1. Because the patient characteristics were calculated on the raw data (before the multiple imputation), the numbers do not 
always add up to a total of 1353 patients. A total of 508 patients (38,3\%) experienced moderate to severe preoperative pain during the week before the surgery, and 818 patients (61,7\%) did not. A small majority was female. Most patients were ASA physical status class I or II. Most procedures were performed by the following specialties: general surgery (395 patients, 29,2\%), orthopaedic surgery $(289,21,4 \%)$ and ear-nosethroat surgery $(204,15,1 \%)$. A list of the included procedures per specialty has been published elsewhere. ${ }^{11}$

Table 1: Patient characteristics for patients with and without moderate to severe preoperative pain.

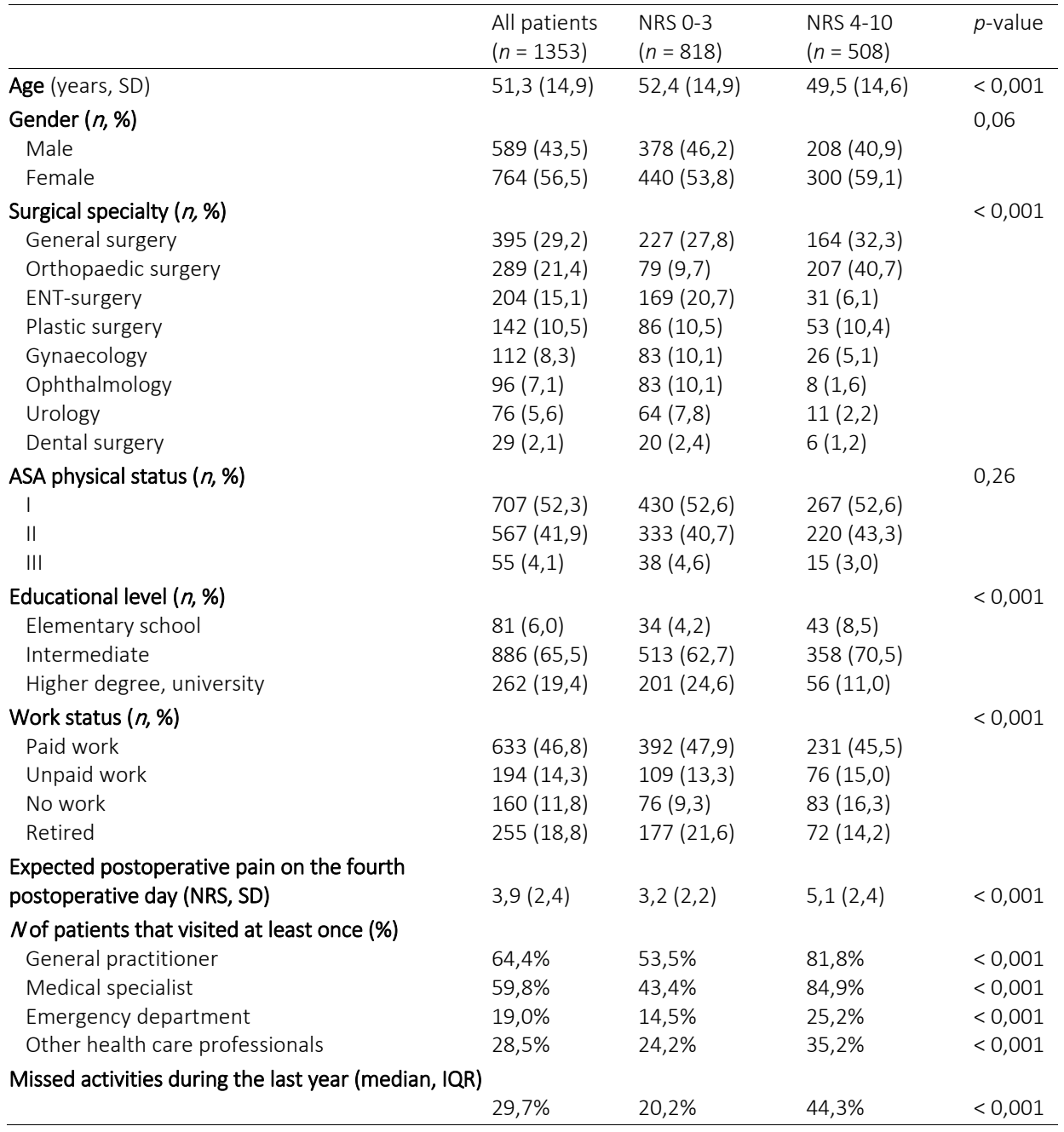

$p$-value demonstrates the significance level of the difference of the preoperative variable between patients with and with moderate to severe preoperative pain. 


\section{Hospital-based health care costs}

For the analyses of this outcome 1353 patients were included. The average amount of hospital-based health care costs during the first postoperative year after outpatient surgery was $€ 1.520$ (Confidence Interval (CI) €1.406-€1.633), with a minimum of €39 and a maximum of $€ 26.920$. The amount of hospital-based health care costs was statistically significantly different between the surgical specialties $(p<0,001)$. Table 2 demonstrates the average amount of health care costs per surgical specialty for all patients and for patients with and without moderate to severe preoperative pain. Most of the costs were created by patients after ENT-surgery (€1.768, Cl €1.337-€2.199), dental surgery (€1.697, Cl €170-€3.224), and orthopaedic surgery (€1.589, Cl €1.372$€ 1.805)$.

Table 2: Average hospital-based health care costs in euros per surgical specialty during the first postoperative year for patient with and without moderate to severe preoperative pain. (NRS = numeric rating scale, $\mathrm{Cl}=$ confidence interval).

\begin{tabular}{lllll}
\hline Surgical specialty & \multicolumn{2}{l}{$\begin{array}{l}\text { Average hospital based health care costs in euros } \\
\text { Mean }(95 \% \mathrm{Cl})\end{array}$} & $p$-value \\
\cline { 2 - 4 } & All patients & NRS 0-3 $(n=832)$ & NRS 4-10 $(n=521)$ & \\
\hline All specialties $(n=1353)$ & $€ 1.520(1.406-1.633)$ & $€ 1.431(1.291-1.570)$ & $€ 1.662(1.468-1.856)$ & 0,001 \\
General surgery $(n=394$ & $€ 1.551(1.346-1.756)$ & $€ 1.651(1.376-1.927)$ & $€ 1.412(1.108-1.717)$ & $0,42-0,56$ \\
Orthopaedic surgery $(n=289)$ & $€ 1.589(1.372-1.805)$ & $€ 1.157(944-1.370)$ & $€ 1.751(1.467-2.035)$ & 0,012 \\
ENT-surgery $(n=204)$ & $€ 1.768(1.337-2.199)$ & $€ 1.584(1.177-1.991)$ & $€ 2.756(1.113-4.400)$ & $<0,01$ \\
Plastic surgery $(n=142)$ & $€ 1.547(1.341-1.753)$ & $€ 1.530(1.275-1.785)$ & $€ 1.574(1.225-1.924)$ & $0,84-0,96$ \\
Gynaecology $(n=112)$ & $€ 1.252(966-1.538)$ & $€ 1.202(860-1.543)$ & $€ 1.417(917-1.916)$ & $0,05-0,06$ \\
Ophthalmology $(n=96)$ & $€ 1.228(905-1.551)$ & $€ 1.264(907-1.621)$ & $€ 947(381-1.514)$ & $0,12-0,40$ \\
Urology $(n=76)$ & $€ 1.005(771-1.238)$ & $€ 873(717-1.029)$ & $€ 1.782(509-3.054)$ & 0,033 \\
Dental surgery $(n=29)$ & $€ 1.697(170-3.224)$ & $€ 1.729(0-3.666)$ & $€ 1.598(0-3.543)$ & $0,48-0,86$ \\
\hline
\end{tabular}

$p$-value demonstrates significance between patients with and without moderate to severe preoperative pain.

The average amount of postoperative hospital-based health care costs was $€ 1.431$ euros ( $\mathrm{Cl} € 1.291-€ 1.570)$ for patients with a preoperative NRS of 0-3 and $€ 1.662(\mathrm{Cl}$ $€ 1.468-€ 1.856)$ for patients with a preoperative NRS of $4-10$. This difference was statistically significant $(p<0,001)$. Linear regression analysis demonstrated that age and work status were confounders, but even with these variables in the model, preoperative pain remained statistically significantly associated with the amount of postoperative hospital-based health care costs. Other preoperative variables (i.e. ASA physical status, educational level, life optimism trait, pain catastrophizing and surgical fear) were not statistically significant confounders.

In patients who underwent orthopaedic surgery, urologic surgery or ENT-surgery the amount of postoperative hospital-based health care costs was statistically significantly different between patients with and without moderate to severe preoperative pain. For these specialties multiple linear regression analyses were performed. In orthopaedic surgery, life optimism trait was a confounder, but even with this confounder in the model, preoperative pain remained associated with postoperative hospital-based health 
care costs. In patients after urologic surgery no confounders were identified. In patients who underwent ENT-surgery, the severity of preoperative pain was no longer significantly associated with the amount of postoperative hospital-based health care costs after addition of the patient's age into the model.

\section{Other health care costs}

Table 3a demonstrates the average amount of visits to health care providers for patients with and without moderate to severe preoperative pain. Table $3 b$ demonstrates the costs associated with these visits. Most patients did not visit their general practitioner ( 840 patients, 62,1\%), a medical specialist (895 patients, 66,1\%), an emergency department $(1193,88,2 \%)$ or other health care professional $(1081,79,9 \%)$ with questions about pain during the first postoperative year. Patients with moderate to severe preoperative pain visited their general practitioner $(p<0,001)$, medical specialist $(p<0,001)$, or other health care professional $(p<0,001)$, significantly more often than patients without moderate to severe preoperative pain. This was also associated with increased costs $(p<0,001)$. The number of visits to an emergency department did not differ between the two groups

\section{Indirect costs}

Table 3a shows the amount of absent days from paid and unpaid work for the two groups. Table 3b shows the indirect costs associated with absenteeism. At the time of the surgical procedure 437 patients $(47,9 \%)$ had a paid job. The median amount of absent days was 0 (IQ-range 0-14). Patients with moderate to severe preoperative pain were significantly more often absent from work than patients without moderate to severe preoperative pain $(p<0,01)$. The average costs associated with absenteeism from a paid job were $€ 4.789$. These costs were significantly higher for patients with moderate to severe preoperative pain (€6.643, 95\% Cl €4.477-€8.809) compared to patients without preoperative pain (€3.646, 95\% Cl €2.497-4.794, $p$ 0,01). Patients with unpaid work (e.g. students, volunteers, etc) at the time of the surgical procedure were not significantly more often absent during the first postoperative year. 
Table 3a: Secondary outcome parameters: percentage of patients that visited health care professional at least once, or were absent from work and regular activities at least once during the first postoperative year. Table also shows the median amount of visits or absent days for patients with at least one visit or one absent day.

\begin{tabular}{|c|c|c|c|}
\hline & $\begin{array}{l}\text { NRS } 0-3 \\
(n=564)\end{array}$ & $\begin{array}{l}\text { NRS } 4-10 \\
(n=348)\end{array}$ & $P$-value \\
\hline \multicolumn{4}{|c|}{$\begin{array}{l}\text { Visits to health care professionals (\% that visited at } \\
\text { least once, median } n \text { of visits for these patients) }\end{array}$} \\
\hline General practitioner & $20,2 \%(2)$ & $36,2 \%(2)$ & $<0,001$ \\
\hline Medical specialist & $17,2 \%(2)$ & $39,1 \%(3)$ & $<0,001$ \\
\hline Emergency department & $6,9 \%(1)$ & $10,1 \%(1)$ & $0,05-0,19$ \\
\hline Other health care professionals & $9,9 \%(13)$ & $25,0 \%(15)$ & $<0,001$ \\
\hline \multicolumn{4}{|c|}{$\begin{array}{l}\text { Absenteeism from work (\% that was absent at least } \\
\text { one day, median } n \text { of days for these patients) }\end{array}$} \\
\hline Paid work & $38,1 \%(14)$ & $50,9 \%(25)$ & $<0,01$ \\
\hline Unpaid work & $2,3 \%(2)$ & $9,7 \%(76)$ & $0,01-0,36$ \\
\hline \multicolumn{4}{|c|}{$\begin{array}{l}\text { Missed activities during the first postoperative year } \\
\text { (\% that missed at least one activity, median } n \text { missed }\end{array}$} \\
\hline activities for these patients) & $13,1 \%(14)$ & $54,1 \%(41)$ & $<0,001$ \\
\hline
\end{tabular}

Table 3b: Secondary outcome parameters: average costs in euros of visits to health care professionals and absenteeism from paid work during the first postoperative year. (NRS = numeric rating scale, $\mathrm{Cl}=$ confidence interval)

\begin{tabular}{llll}
\hline & $\begin{array}{l}\text { NRS 0-3 } \\
(n=564)\end{array}$ & $\begin{array}{l}\text { NRS 4-10 } \\
(n=348)\end{array}$ & $P$-value \\
\hline $\begin{array}{l}\text { Costs of visits during the first } \\
\text { postoperative year (costs, 95\% Cl) }\end{array}$ & & & \\
$\begin{array}{l}\text { General practitioner } \\
\text { Medical specialist }\end{array}$ & $€ 16(19-27)$ & $€ 33(25-40)$ & $<0,001$ \\
$\begin{array}{l}\text { Emergency department } \\
\text { Other health care professionals }\end{array}$ & $€ 13(9-87)$ & $€ 227(164-289)$ & $<0,001$ \\
$\begin{array}{l}\text { Costs of absenteeism from paid work } \\
\text { (costs, 95\% Cl) }\end{array}$ & $€ 3.646(2.497-413)$ & $€ 19(12-27)$ & $0,05-0,19$ \\
\hline
\end{tabular}

\section{Discussion}

Our study demonstrates for the first time, that the severity of preoperative pain is associated with an increased amount of hospital-based health care costs during the first year after outpatient surgery. As it is reported that the severity of preoperative pain is one of the most important risk factors for the development of chronic postsurgical pain (CPSP), ${ }^{11-14}$ it is far from unlikely to assume that patients with CPSP use more health care resources and thus generate more health care costs than patients without CPSP. A study by Parsons et al. demonstrated that patients with more severe post-trauma or post-surgical neuropathic pain create higher direct and indirect postoperative expenditures. ${ }^{9}$ The average annual calculated costs in this study were much higher than the average costs in our study. This difference can be explained by differences in the 
structure of health care systems (i.e. The Netherlands versus the United States) and by underreporting of costs in our study. For example, costs generated by prescription of medications were not measured in our study, but a significant percentage of the total costs in the study by Parsons et al was due to prescription medication. ${ }^{9}$

From our results we conclude that the association between the severity of preoperative pain and the amount of hospital-based health care costs was most clear for patients after orthopaedic and urologic surgery. Previous studies have shown that orthopaedic surgery is a risk factor for the development of acute and chronic postoperative pain. ${ }^{11,24}$ The prevalence of moderate to severe preoperative pain was much higher in this group of orthopaedic patients as compared to the total population $(72,7 \%$ versus $38,3 \%)$. In many of these patients the severity of preoperative pain is the reason to perform the surgical procedure. We hypothesize that in many of these orthopaedic patients the pre-existing pain continues into the postoperative phase, due to failure of the surgical procedure to relief this pain (e.g. knee arthroscopy). In addition to this, many of these patients undergo an expensive second surgical procedure during the first postoperative year (e.g. total knee replacement). This might account for the high amount of health care costs after orthopaedic surgery. Future studies are necessary to confirm these results and will hopefully be able to identify other risk factors for high hospital-based health care costs after orthopaedic surgery.

Future studies are needed to confirm preoperative pain as a risk factor for increased postoperative health care costs, as well as to identify other risk factors. The next step will be to determine if it is possible to reduce these costs by optimizing preoperative risk factors. Optimizing treatment of preoperative pain, might lead to a reduction in postoperative health care costs, as well as other negative outcomes (e.g. the prevalence CPSP). This might be achieved by conventional analgesics, but also via treatment of psychological risk factors and by improving preoperative patient education. In this respect it is interesting to note that preoperative neuroscience education by a physical therapist, in patients with preoperative pain due to radiculopathy, resulted in less health care expenditure during the first year after lumbar surgery as compared to routine preoperative care. ${ }^{15} \mathrm{~A}$ study to evaluate the effect of mindfulness training prior to hip or knee arthroplasty on physical function and postoperative health care consumption is being performed. ${ }^{25}$ No studies are available that test the association between the optimization of conventional analgesics during the preoperative period and postoperative health care expenditures.

Our study has several limitations. First of all, the calculated amount of hospitalbased health costs was based on the procedures and visits performed in our own hospital. It is unknown if patients visited other hospitals during the first year after the surgery. Because our hospital is a large university hospital, where patients from a large area are treated, it is possible that patients visited smaller local hospitals for other medical problems. This might lead to underreporting of the actual use of health care facilities and thus to lower calculated overall hospital-based health care costs. In case of 
future studies, data should be collected via the patients insurance companies in order to overcome this limitation. A second limitation is the self-reporting of the number of visits to their general practitioner, medical specialist, emergency department or other health care professional during the last year. Previous studies have shown that the validity of self-reported visits to outpatient clinics is low. ${ }^{26-28}$ Whether the accuracy of the self-reported number of visits decreases with time is controversial. ${ }^{26,27}$ Even though self-reported number of visits might seem more accurate with shorter recall periods, this is probably not due to more accurate reporting, but rather related to relatively more over-reporting and less under-reporting. ${ }^{27}$

In conclusion, our study demonstrates that the severity of preoperative pain is statistically significantly associated with the amount of hospital-based health care costs during the first year after outpatient surgery. This association is strongest for patients after orthopaedic or urologic surgery. Patients with moderate to severe preoperative pain visited their general practitioner, medical specialist or other health care professional more often, than patients with mild to no pain. This study demonstrates that it is of utmost importance to treat preoperative pain. Future studies will have to be performed in order to determine which preoperative interventions (e.g. optimizing analgesic therapy, psychological interventions, etc). will be able to reduce postoperative health care expenditures in patients with preoperative pain. 


\section{References}

1. Schaefer C, Sadosky A, Mann R, et al. Pain severity and the economic burden of neuropathic pain in the United States: BEAT Neuropathic Pain Observational Study. ClinicoEconomics and outcomes research : CEOR 2014; 6: 483-96.

2. Nickel R, Raspe HH. [Chronic pain: epidemiology and health care utilization]. Der Nervenarzt 2001; 72(12): 897-906.

3. Breivik H, Collett B, Ventafridda V, Cohen R, Gallacher D. Survey of chronic pain in Europe: prevalence, impact on daily life, and treatment. Eur J Pain 2006; 10(4): 287-333.

4. Liedgens H, Obradovic M, De Courcy J, Holbrook T, Jakubanis R. A burden of illness study for neuropathic pain in Europe. ClinicoEconomics and outcomes research : CEOR 2016; 8: 113-26.

5. Juniper M, Le TK, Mladsi D. The epidemiology, economic burden, and pharmacological treatment of chronic low back pain in France, Germany, Italy, Spain and the UK: a literature-based review. Expert opinion on pharmacotherapy 2009; 10(16): 2581-92.

6. Kehlet $\mathrm{H}$, Jensen TS, Woolf CJ. Persistent postsurgical pain: risk factors and prevention. Lancet 2006; 367(9522): 1618-25.

7. Simanski CJ, Althaus A, Hoederath S, et al. Incidence of chronic postsurgical pain (CPSP) after general surgery. Pain Med 2014; 15(7): 1222-9.

8. Lavand'homme P. The progression from acute to chronic pain. Curr Opin Anaesthesiol 2011; 24(5): 54550.

9. Parsons B, Schaefer C, Mann R, et al. Economic and humanistic burden of post-trauma and post-surgical neuropathic pain among adults in the United States. J Pain Res 2013; 6: 459-69.

10. Hollisaaz MT, Noorbala MH, Irani N, et al. Severity of chronic pain affects health care utilization after kidney transplantation. Transplant Proc 2007; 39(4): 1122-5.

11. Hoofwijk DM, Fiddelers AA, Peters ML, et al. Prevalence and Predictive Factors of Chronic Postsurgical Pain and Poor Global Recovery One Year after Outpatient Surgery. Clin J Pain 2015.

12. Macrae WA. Chronic post-surgical pain: 10 years on. Br J Anaesth 2008; 101(1): 77-86.

13. Sommer M, Geurts JW, Stessel B, et al. Prevalence and predictors of postoperative pain after ear, nose, and throat surgery. Archives of otolaryngology--head \& neck surgery 2009; 135(2): 124-30.

14. Katz J, Seltzer Z. Transition from acute to chronic postsurgical pain: risk factors and protective factors. Expert Rev Neurother 2009; 9(5): 723-44.

15. Louw A, Diener I, Landers MR, Puentedura EJ. Preoperative pain neuroscience education for lumbar radiculopathy: a multicenter randomized controlled trial with 1-year follow-up. Spine 2014; 39(18): 1449-57.

16. Sullivan MJ, Bishop SR. The Pain Catastrophizing Scale: Development and Validation. Psychological Assessment 1995; 7(4): 524-32.

17. Scheier MF, Carver CS, Bridges MW. Distinguishing optimism from neuroticism (and trait anxiety, selfmastery, and self-esteem): a reevaluation of the Life Orientation Test. J Pers Soc Psychol 1994; 67(6): 1063-78.

18. Theunissen $M$, Peters ML, Schouten EG, et al. Validation of the surgical fear questionnaire in adult patients waiting for elective surgery. PloS one 2014; 9(6): e100225.

19. van Agt HM, Essink-Bot ML, Krabbe PF, Bonsel GJ. Test-retest reliability of health state valuations collected with the EuroQol questionnaire. Soc Sci Med 1994; 39(11): 1537-44.

20. Peters ML, Sommer M, van Kleef M, Marcus MA. Predictors of physical and emotional recovery 6 and 12 months after surgery. Br J Surg 2010; 97(10): 1518-27.

21. Hakkaart L, Tan SS, Bouwmans CAM. Handleiding voor kostenonderzoek, methoden en standaard kostprijzen voor economische evaluaties in de gezondheidszorg: College voor zorgverzekeringen (CVZ); Geactualiseerde versie 2010. 
22. Gerbershagen HJ, Rothaug J, Kalkman CJ, Meissner W. Determination of moderate-to-severe postoperative pain on the numeric rating scale: a cut-off point analysis applying four different methods. Br J Anaesth 2011; 107(4): 619-26.

23. Dihle A, Helseth S, Paul SM, Miaskowski C. The exploration of the establishment of cutpoints to categorize the severity of acute postoperative pain. Clin J Pain 2006; 22(7): 617-24.

24. Gramke HF, de Rijke JM, van Kleef $M$, et al. Predictive factors of postoperative pain after day-case surgery. Clin J Pain 2009; 25(6): 455-60.

25. Dowsey MM, Castle DJ, Knowles SR, Monshat K, Salzberg MR, Choong PF. The effect of mindfulness training prior to total joint arthroplasty on post-operative pain and physical function: study protocol for a randomised controlled trial. Trials 2014; 15: 208.

26. Bellon JA, Lardelli P, Luna JD, Delgado A. Validity of self reported utilisation of primary health care services in an urban population in Spain. Journal of epidemiology and community health 2000; 54(7): 544-51.

27. Icks A, Dittrich A, Brune $M$, et al. Agreement found between self-reported and health insurance data on physician visits comparing different recall lengths. J Clin Epidemiol 2017; 82: 167-72.

28. Wolinsky FD, Miller TR, An H, et al. Hospital episodes and physician visits: the concordance between selfreports and medicare claims. Medical care 2007; 45(4): 300-7. 


\section{Chapter}

Genetic polymorphisms and their association with the prevalence and severity of chronic postsurgical pain - A systematic review 


\section{Abstract}

\section{Background}

Although several demographic, clinical and psychological risk factors for chronic postsurgical pain (CPSP) have been identified, genetic variants including single nucleotide polymorphisms have also become of interest as potential risk factors for the development of CPSP. The aim of this review is to summarize the current evidence on genetic polymorphisms associated with the prevalence and severity of CPSP in adult patients.

\section{Methods}

A systematic review of the literature was performed, and additional literature was obtained by reference tracking. The primary outcome was CPSP, defined as pain at least two months after the surgery. Studies performed exclusively in animals were excluded.

\section{Results}

Out of the 1001 identified studies, 14 studies were selected for inclusion. These studies described 5269 participants in 17 cohorts. A meta-analysis was not possible because of heterogeneity of data and data analysis. Associations with the prevalence or severity of CPSP were reported for genetic variants in the COMT gene, OPRM1, potassium channel genes, GCH1, CACNG, CHRNA6, P2X7R, cytokine associated genes, HLA, DRD2, and ATXN1.

\section{Discussion}

Research on the topic of genetic variants associated with CPSP is still in its initial phase. Hypothesis-free, genome-wide association studies on large cohorts are needed in this field. In addition, future studies may also integrate genetic risk factors and patient characteristics, clinical and psychological predictors for CPSP. 


\section{Introduction}

Chronic postsurgical pain (CPSP) is a common problem that can occur after any type of surgery. ${ }^{1,2}$ The prevalence of moderate to severe CPSP varies between 10 and $60 \% .{ }^{1,3,4}$ Chronic postsurgical pain not only has a negative influence on the quality of life of the patient, but it also has major socioeconomic consequences. ${ }^{5,6}$ Several studies have been performed to determine the risk factors for the development of CPSP, and a number of patient characteristic, clinical and psychological risk factors have already been identified. ${ }^{1,2,7-9}$ However, these studies have also demonstrated that a substantial amount of the variance in CPSP cannot be explained by these predictors. ${ }^{1,2,9}$

Recently, polymorphisms of several genes have also become of interest as potential risk factors for the prevalence and severity of CPSP. It is suspected that some genes predispose to CPSP whereas others are associated with a protective effect. Data from twin studies and human pedigrees estimate that the heritability of chronic pain ranges from 30 to $70 \% .{ }^{10}$ Research has focused on variants in genes that encode for proteins involved in nerve conduction and transmission, opioid receptor signalling and inflammatory processes. ${ }^{11-15}$

The lack of ability of patient characteristic, clinical and psychological factors to explain all the variance in the prevalence and severity of CPSP, coupled with the interest in genetic polymorphisms as predictors of other chronic pain states, leads to two questions. Are genetic polymorphisms associated with the prevalence and severity of CPSP? If this is the true, does adding these polymorphisms to the existing prediction models improve their accuracy?

Therefore, the objective of this systematic review is to summarize the current evidence with respect to genetic polymorphisms as predictors for the prevalence (i.e. the number of patients in the study population that develop CPSP) and severity of CPSP after any type of surgery in adult patients. Finding consistent and reliable genetic predictors might improve the understanding of the molecular mechanisms underlying the development of CPSP and reveal therapeutic targets, and might also improve preoperative prediction models and thus offer surgeons and anaesthetists the possibility to identify patients at risk for developing CPSP.

\section{Methods}

\section{Search strategy}

This review was performed according to the Preferred Reporting Items for Systematic reviews and Meta-Analysis (PRISMA) guidelines. A systematic literature review was conducted, using the databases of PubMed, Embase and PsychINFO. The final search 
strategy was as follows: ("Pain, Postoperative"[Mesh] OR ("persistent pain" AND surgery) OR ("chronic postsurgical pain") OR ("chronic post surgical pain") OR cpsp OR ("persistent postoperative pain") OR ("chronic postoperative pain") OR (“chronic pain" AND surgery)) AND (genetic* OR gene OR genes OR snp OR snps OR polymorphism*) NOT "pain treatment" NOT "pain therapies" NOT "pain management" NOT "pain control". Electronic searches were limited to the availability of abstracts, and to studies published in the English, French, German or Dutch language. Additional searches were carried out by reference tracking and expert consultation.

\section{Study selection and inclusion criteria}

Studies eligible for inclusion were observational cohort studies, case-control studies and randomized controlled trials, performed in adult patients undergoing any type of surgical procedure. Conference abstracts and case reports were excluded, as were studies performed exclusively in animals. Studies were included if the study population had already undergone surgery and the follow-up time was at least two months. This time frame was chosen in line with the definition of CPSP. ${ }^{16}$ Pain had to be the primary outcome parameter, but the method by which pain was measured was not a specific inclusion or exclusion criterion. All included studies had to measure genetic polymorphisms directly. Indirect measurement, via the analysis of gene products, resulted in exclusion of the study. The abstracts retrieved from the search were all read and assessed independently by two authors (D.M.N.H. and R.R.I.v.R.). If an abstract fulfilled all inclusion criteria, or if any aspect of the abstract was unclear, the full text was obtained and assessed by the two reviewers (D.M.N.H. and R.R.I.v.R.). Differences in opinion were resolved through structured discussion.

\section{Data extraction and data analysis}

The following information was extracted from each study: reporting author, publication year, study design, number of participants, ethnicity of participants, type of surgery, primary and secondary outcome measures, follow-up time, polymorphisms studied, and type of statistical analyses used. The quality of the studies was assessed using an eightitem checklist (scores 0-8), based on the Critical Appraisal Checklist for Cohort/Case Control of the Joanna Briggs institute, ${ }^{17}$ and the checklist for measuring study quality developed by Downs and Black. ${ }^{18}$ The quality of each study was assessed independently by two authors (D.M.N.H. and R.R.I.v.R.) This eight-item checklist was used in previous research, where substantial agreement between raters was demonstrated. ${ }^{8}$ An explanation of this quality checklist is available elsewhere. ${ }^{8}$ We considered performing a meta-analysis for polymorphisms that were reported by more than one study. Unfortunately, an accurate and reliable meta-analysis could not be performed because only a few polymorphisms were reported by more than one study. Furthermore, study 
design (e.g. included surgical procedures, definition of CPSP, follow-up time), statistical analysis, and the manner of reporting the results (e.g. odds ratios (ORs) versus only $p$ values) varied too much between authors.

\section{Results}

The systematic search and reference tracking resulted in 1002 publications (Figure 1). Of these initial 1002 articles, 49 duplicates were removed and 920 articles were excluded after screening of the abstracts. Most of these 920 articles were either conference abstracts or animal studies. Hence, a total of 33 full-text articles were screened, of which 18 were excluded (in four articles pain was not an outcome parameter, 12 articles did not measure CPSP, two articles did not include genetic analyses, and one article included patients younger that 18 years old). A total of 14 articles, including 5269 participants in 17 cohorts, were finally used for the analyses.

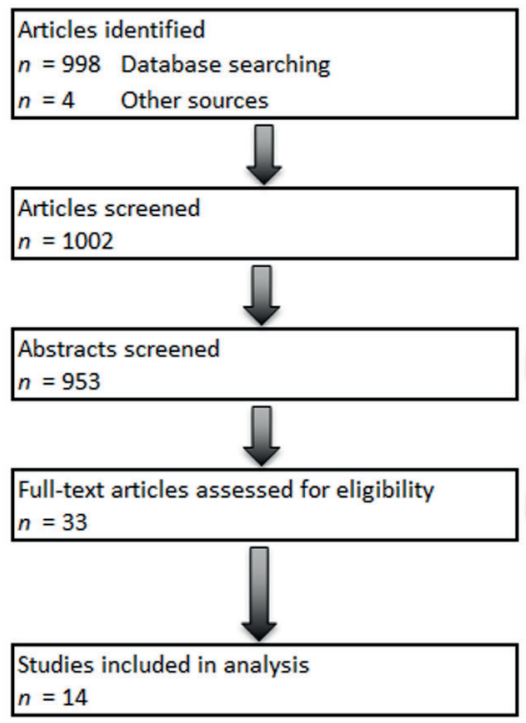

Duplicates removed

$n=49$

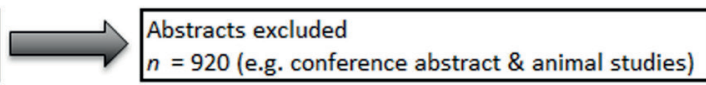

Figure 1: Flowchart of the process of article selection.

The characteristics of the patient cohorts are summarized in Table 1. The most common surgical procedures were breast surgery and inguinal hernia repair. In all studies CPSP was measured at three months or more after the surgical procedure. Seven of the cohorts studied a Caucasian population, three cohorts studied an Israeli population (of Ashkenazi and non-Ashkenazi descent), one cohort studied a Chinese population and the remaining studies included participants of diverse ethnicities or did not report the ethnicity of the participants. The sample size of the included cohorts varied between 42 
and 1005 participants. The relatively low sample size of many studies, combined with the number of polymorphisms investigated, resulted in low statistical power in most studies. Moreover, the statistical analyses used in the studies differed considerably; one study used multiple testing corrections by the Bonferroni method, ${ }^{11}$ two other studies chose not to correct for multiple testing because they considered their investigation to be of exploratory nature, and the remaining studies did not report whether they performed multiple testing correction. ${ }^{13,15}$ The quality of the studies varied between four and seven points on the eight-item quality checklist. Most points were lost because of the quality of the statistical analyses (i.e. lack of statistical power), and because of data dredging (i.e. retrospective studies).

Table 2 lists the results of each cohort, including the association between the studied polymorphisms and the prevalence or severity of CPSP. Only polymorphisms that were reported as significantly associated with CPSP in at least one study are mentioned in Table 2. A complete table of all polymorphisms studied is available as additional content (supplemented data). Only statistically significant results will be mentioned in the text. Numerical outcomes, effect sizes, confidence intervals ( $\mathrm{Cl}^{\prime} \mathrm{s}$ ) and information on multiple testing correction are listed in the text if they were mentioned in the original studies. Unfortunately, many studies did not provide numerical outcomes, but stated only whether an association between the polymorphism and prevalence or severity of CPSP was found or not.

\section{COMT}

The COMT gene encodes for the catechol-o-methyl transferase enzyme, which is involved in the inactivation of catecholamines. ${ }^{19,} 20$ Polymorphisms in the COMT gene in relation to CPSP, have been studied in six investigations. ${ }^{11,21-25}$ Two studies reported a significant association between polymorphisms in the COMT gene and CPSP. ${ }^{21,25}$ The minor allele of rs4818 ( $p=0.031)$ and rs4633 ( $p=0.01)$ and the major alleles of rs6269 ( $p$ $=0.02)$ and $r$ 4680 ( $p=0.018)$ were associated with an increased severity of CPSP in a Caucasian population 12 months after lumbar discectomy. ${ }^{21}$ This difference was reported as statistically significant only for pain measured by the 6-point Likert scale used in the Oswestry Disability Index, and not when measured by the Visual Analogue Scale (VAS). The high pain sensitivity haplotype (rs6269A, rs4633C, rs4818C, rs4680G) as described by Diatchenko and colleagues ${ }^{26}$ was associated with lower VAS scores 12 months after lumbar discectomy (OR 1.45, $p=0.046) .^{21}$ This was in contrast to previous findings in experimental pain studies, but the authors did not offer an explanation for these findings. ${ }^{21}$ Furthermore, an association was reported between COMT diplotype (rs4633 and rs4818), pain catastrophizing scale and the severity of CPSP, measured at three, four and five months after arthroscopic shoulder surgery. ${ }^{25}$ Patients with low COMT activity (average and high pain sensitivity diplotype) and high pain catastrophizing scale scores experienced higher pain ratings than patients with other combinations. 


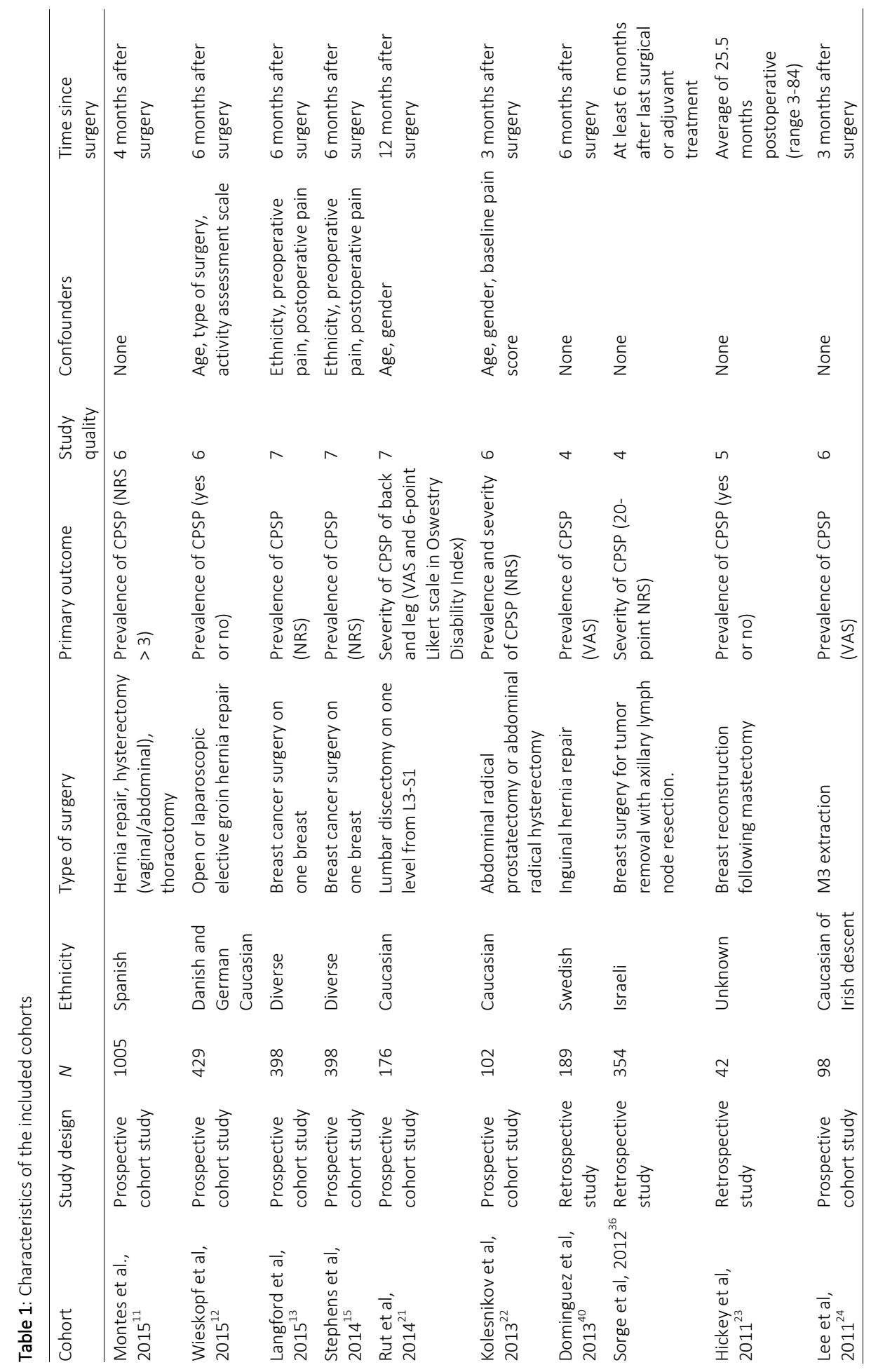




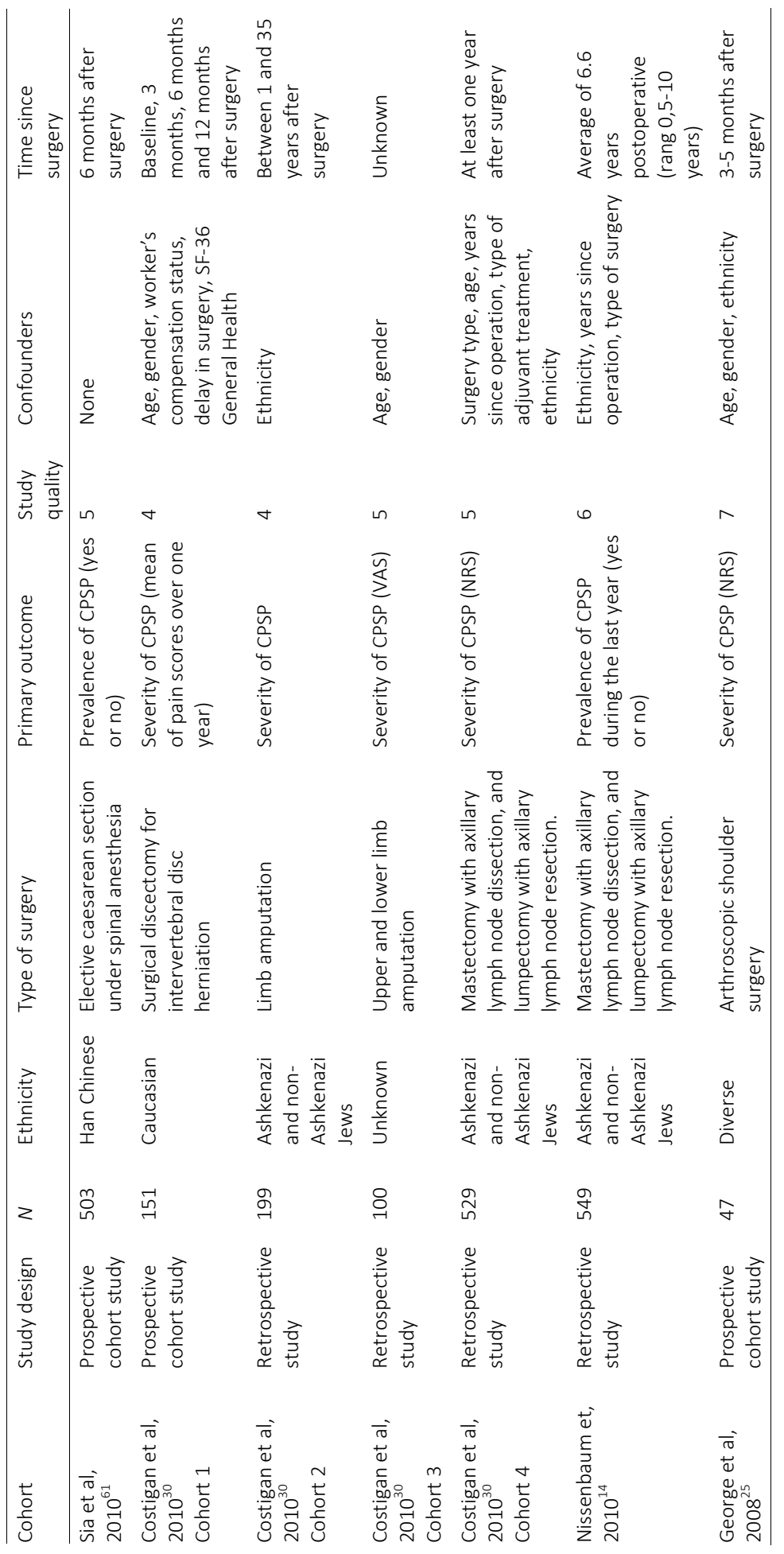


Table 2: Results of the individual studies. The table demonstrates the effect of the minor allele of the polymorphism on the prevalence and/or the severity of chronic postsurgical pain (CPSP). Genes without statistically significant associations were omitted. $+=$ minor allele associated with more CPSP, $-=$ minor allele associated with less CPSP, $0=$ no effect was found, Yes = effect found, but directionality unknown. 0/+= assocation only before multiple testing correction.

\begin{tabular}{|c|c|c|c|c|c|c|c|c|c|c|c|c|c|c|c|c|c|c|}
\hline & & 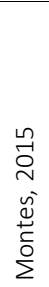 & 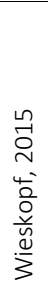 & 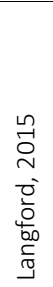 & 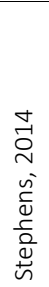 & 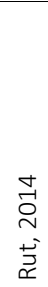 & 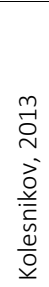 & 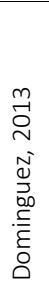 & 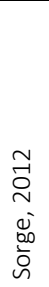 & 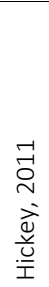 & 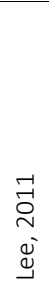 & 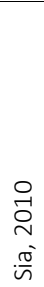 & 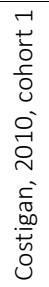 & 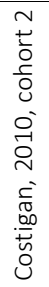 & 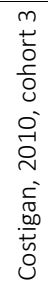 & 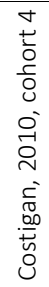 & 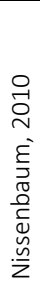 & 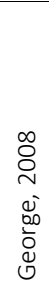 \\
\hline & & 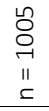 & $\begin{array}{l}\underset{\sim}{\sim} \\
\| \\
\simeq \\
\simeq\end{array}$ & $\begin{array}{c}\infty \\
\underset{m}{\infty} \\
\| \\
\simeq\end{array}$ & $\begin{array}{l}\infty \\
\text { } \\
\text { I } \\
\simeq \\
\simeq\end{array}$ & $\begin{array}{c}0 \\
\underset{1}{1} \\
\text { II } \\
\simeq\end{array}$ & 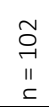 & $\begin{array}{l}\infty \\
\infty \\
\\
\| 1 \\
\subset\end{array}$ & $\begin{array}{l}\stackrel{+}{\mathrm{n}} \\
\stackrel{2}{\prime \prime} \\
\subseteq\end{array}$ & $\begin{array}{l}\stackrel{\sim}{\Im} \\
11 \\
ᄃ \\
\end{array}$ & $\begin{array}{l}\infty \\
0 \\
11 \\
\subset\end{array}$ & $\begin{array}{l}n \\
\text { 으 } \\
\text { II } \\
ᄃ\end{array}$ & 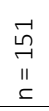 & \begin{tabular}{l} 
ब \\
$\underset{1}{11}$ \\
\multicolumn{1}{c}{}
\end{tabular} & \begin{tabular}{c}
$\bigcirc$ \\
$\stackrel{\bigcirc}{1}$ \\
II \\
\multicolumn{1}{c}{}
\end{tabular} & $\begin{array}{c}\text { N } \\
\text { กิ } \\
\text { II } \\
\simeq\end{array}$ & $\begin{array}{l}\text { g } \\
\text { ஸn } \\
11 \\
ᄃ\end{array}$ & \begin{tabular}{l}
\multirow{f}{f}{} \\
$\|$ \\
$\check{c}$
\end{tabular} \\
\hline \multirow[t]{6}{*}{ COMT } & rs4818 & & & & & + & & & & & 0 & & & & & & & \\
\hline & rs6269 & 0 & & & & - & & & & & 0 & & & & & & & \\
\hline & rs4680 & 0 & & & & - & 0 & & & 0 & 0 & & & & & & & \\
\hline & rs4633 & & & & & + & & & & & & & & & & & & \\
\hline & $\begin{array}{l}\text { Haplotype } \\
\text { Diatchenko }\end{array}$ & & & & & - & & & & & & & & & & & & \\
\hline & Haplotype $1^{\#}$ & & & & & & & & & & & & & & & & & Yes \\
\hline OPRM1 & rs1799971 & 0 & & & & & + & & & & 0 & & & & & & & \\
\hline KCNA1 & rs4766311 & & & - & & & & & & & & & & & & & & \\
\hline \multirow[t]{5}{*}{ KCND2 } & rs17376373 & & & - & & & & & & & & & & & & & & \\
\hline & rs702414 & & & Yes & & & & & & & & & & & & & & \\
\hline & rs802340 & & & Yes & & & & & & & & & & & & & & \\
\hline & rs12706292 & & & Yes & & & & & & & & & & & & & & \\
\hline & rs1072198 & & & + & & & & & & & & & & & & & & \\
\hline \multirow[t]{2}{*}{ KCNS1 } & rs734784 & & & Yes & & & & & & & & & + & + & + & 0 & & \\
\hline & rs13043825 & & & & & & & & & & & & + & 0 & & & & \\
\hline \multirow[t]{9}{*}{ KCNJ3 } & rs6435329 & & & Yes & & & & & & & & & & & & & & \\
\hline & rs11895478 & & & Yes & & & & & & & & & & & & & & \\
\hline & rs3106653 & & & Yes & & & & & & & & & & & & & & \\
\hline & rs3111006 & & & Yes & & & & & & & & & & & & & & \\
\hline & rs12471193 & & & Yes & & & & & & & & & & & & & & \\
\hline & rs7574878 & & & Yes & & & & & & & & & & & & & & \\
\hline & rs12995382 & & & - & & & & & & & & & & & & & & \\
\hline & rs13398937 & & & Yes & & & & & & & & & & & & & & \\
\hline & rs2591157 & & & Yes & & & & & & & & & & & & & & \\
\hline
\end{tabular}




\begin{tabular}{|c|c|c|c|c|c|c|c|c|c|c|c|c|c|c|c|c|c|c|}
\hline & & 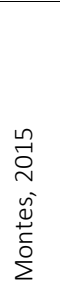 & 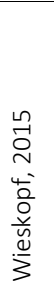 & 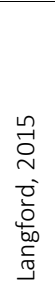 & 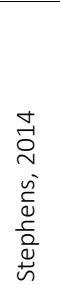 & 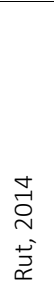 & 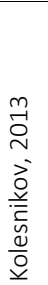 & 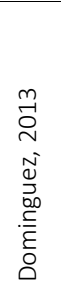 & 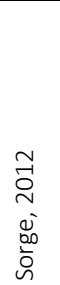 & 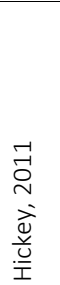 & 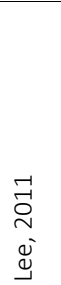 & $\begin{array}{l}0 \\
\text { O } \\
i \\
\text { i⿱ } \\
\text { in }\end{array}$ & 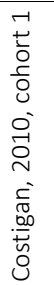 & 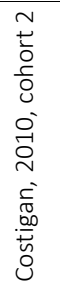 & 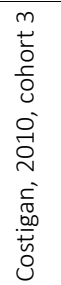 & 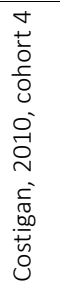 & 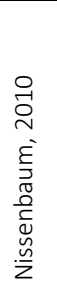 & 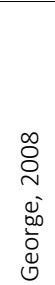 \\
\hline & & 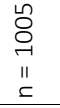 & $\begin{array}{l}\underset{\mathcal{Y}}{\Im} \\
\text { II } \\
=\end{array}$ & $\begin{array}{c}\infty \\
m_{11} \\
\simeq\end{array}$ & $\begin{array}{c}\infty \\
\underset{m}{1} \\
\| \\
ᄃ\end{array}$ & $\begin{array}{l}0 \\
\stackrel{1}{\prime} \\
\prime 1 \\
ᄃ\end{array}$ & 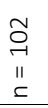 & $\begin{array}{l}\infty \\
\infty \\
\stackrel{1}{1} \\
\simeq \\
\subset\end{array}$ & \begin{tabular}{l}
$\stackrel{+}{\mathrm{n}}$ \\
II \\
\multicolumn{1}{c}{}
\end{tabular} & 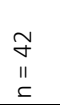 & $\begin{array}{l}\infty \\
o \\
11 \\
\subset\end{array}$ & $\begin{array}{l}m \\
\stackrel{0}{0} \\
\text { II } \\
ᄃ\end{array}$ & $\begin{array}{l}\stackrel{\vec{n}}{\rightarrow} \\
\text { "I } \\
=\end{array}$ & $\begin{array}{l}\stackrel{9}{2} \\
7 \\
11 \\
ᄃ\end{array}$ & \begin{tabular}{l}
$\underset{1}{8}$ \\
II \\
\multicolumn{1}{c}{}
\end{tabular} & $\begin{array}{l}\stackrel{\curvearrowright}{ } \\
\text { N } \\
\text { II } \\
\subset\end{array}$ & $\begin{array}{c}\stackrel{g}{\mathrm{H}} \\
\mathrm{N} \\
\mathrm{II} \\
\subset\end{array}$ & 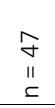 \\
\hline & rs17641121 & & & + & & & & & & & & & & & & & & \\
\hline & rs4467223 & & & Yes & & & & & & & & & & & & & & \\
\hline & Haplotype $2^{\#}$ & & & - & & & & & & & & & & & & & & \\
\hline KCNJ6 & rs860795 & & & Yes & & & & & & & & & & & & & & \\
\hline & rs857967 & & & Yes & & & & & & & & & & & & & & \\
\hline & rs858010 & & & Yes & & & & & & & & & & & & & & \\
\hline & rs858003 & & & + & & & & & & & & & & & & & & \\
\hline & rs2835914 & & & Yes & & & & & & & & & & & & & & \\
\hline & rs858035 & & & Yes & & & & & & & & & & & & & & \\
\hline & rs2835925 & & & + & & & & & & & & & & & & & & \\
\hline KCNK3 & rs1662988 & & & Yes & & & & & & & & & & & & & & \\
\hline & rs7584568 & & & Yes & & & & & & & & & & & & & & \\
\hline KCNK9 & rs 2542424 & & & - & & & & & & & & & & & & & & \\
\hline & rs2014712 & & & + & & & & & & & & & & & & & & \\
\hline & rs2545457 & & & + & & & & & & & & & & & & & & \\
\hline & rs888349 & & & Yes & & & & & & & & & & & & & & \\
\hline $\mathrm{GHC1}$ & rs4411417 & $0 /+$ & & & & & & & & & & & & & & & & \\
\hline CACNG & rs4820242 & & & & & & & & & & & & & & & & + & \\
\hline & rs2284015 & & & & & & & & & & & & & & & & + & \\
\hline & rs2284017 & & & & & & & & & & & & & & & & + & \\
\hline & rs2284018 & & & & & & & & & & & & & & & & + & \\
\hline & rs1883988 & & & & & & & & & & & & & & & & + & \\
\hline & Haplotype $3^{\#}$ & & & & & & & & & & & & & & & & + & \\
\hline CHRNA6 & rs7828365 & & + & & & & & & & & & & & & & & & \\
\hline$P 2 X 7 R$ & rs208294 & & & & & & & & + & & & & & & & & & \\
\hline & rs208296 & & & & & & & & - & & & & & & & & & \\
\hline & rs7958311 & & & & & & & & - & & & & & & & & & \\
\hline IFNG1 & rs2069727 & & & & Yes & & & & & & & & & & & & & \\
\hline & rs2069718 & & & & Yes & & & & & & & & & & & & & \\
\hline
\end{tabular}




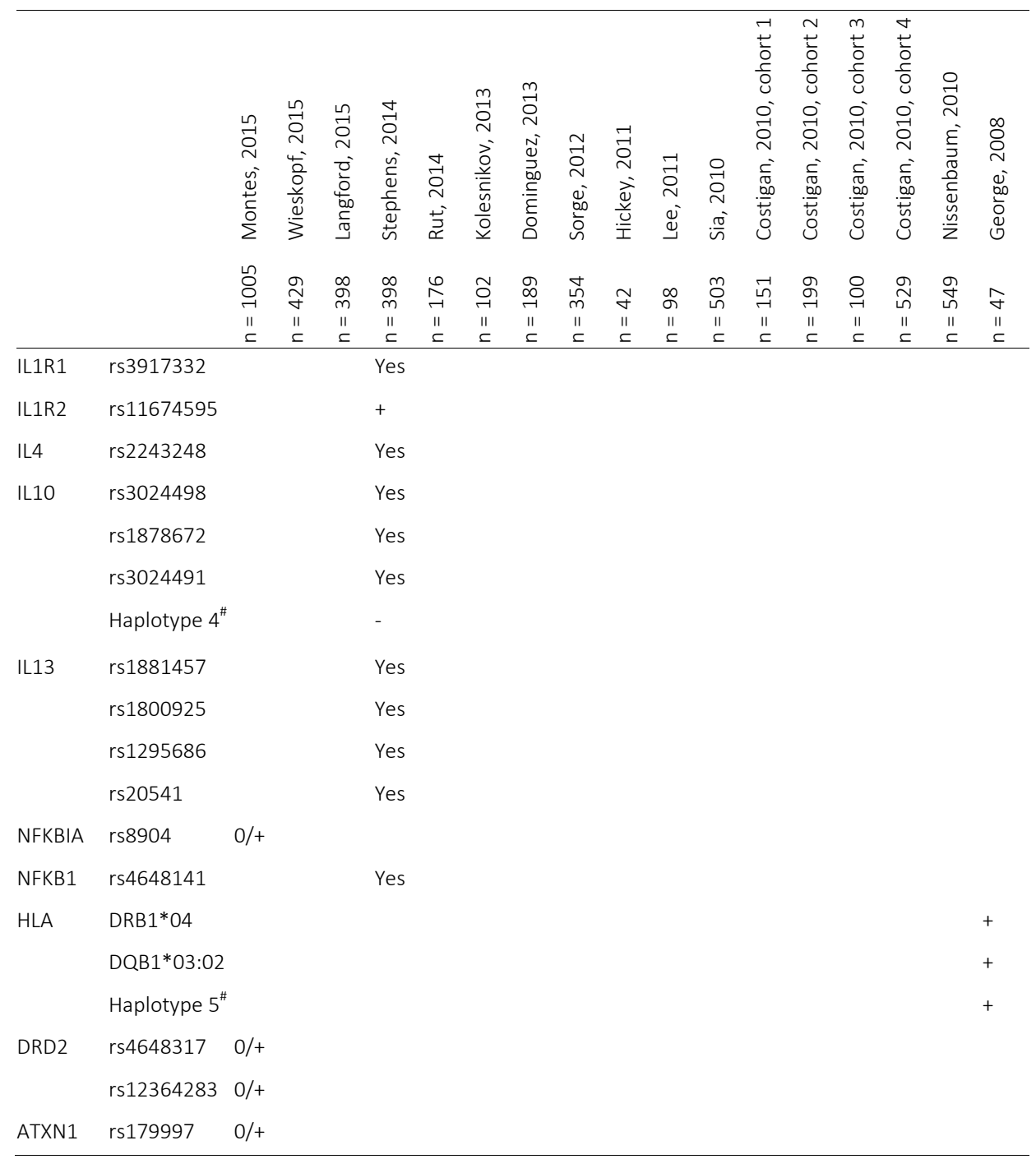

"Haplotype of Diatchenko: rs6269A, rs4633C, rs4818C, rs4680G; "Haplotype 1: rs4633C, rs4818C; "Haplotype 2: rs3111020G, rs11895478A; "Haplotype 3: rs4820242A, rs2284015C, rs2284015C; "Haplotype 4: rs3024505C, rs3024498A, rs3024496T, rs1878672C, rs1518111A, rs1518110T, rs3024491G; "Haplotype 5: DRB1*04, DQB1*03:02.

\section{Opioid receptors}

Three families of opioid receptors $(\mu, \kappa$, and $\delta$ ) have been identified and have been investigated in the context of CPSP. ${ }^{27}$ The most extensively studied receptor is the $\mu$ opioid receptor, which is encoded by the OPRM1 gene. The importance of this receptor in mediating the actions of endogenous opioids, morphine and other morphine-like drugs has been well established. ${ }^{28}$ The association between two polymorphisms of the 
OPRM1 gene (rs1799971 and rs563649) and the prevalence of CPSP has been studied in three patient cohorts. ${ }^{11,22,24}$ Carriers of at least one copy of the minor allele of rs1799971 experienced increased pain scores three months after abdominal surgery as compared to patients homozygous for the major allele $(p=0.02)$, even though the prevalence of CPSP was not increased $(p=0.22)$. The other cohorts did not show an association between polymorphisms of the OPRM1 gene and the prevalence or severity of CPSP. ${ }^{11,24}$ Polymorphisms of the OPRD1 and OPRK1 gene were studied in only one study and no association with the prevalence of CPSP was found. ${ }^{11}$

\section{Potassium channels}

Potassium channels are a vital part of the action potential and may influence pain conduction and transmission. They are tetrameric proteins, of which each subunit may harbour structural differences based on genetic variants. ${ }^{29}$ Structurally different subunits, encoded by seven different genes, have been studied in relation to CPSP (KCNA1, KCND2, KCNS1, KCNJ3, KCNJ6, and KCNK9). ${ }^{13,30}$ A statistically significant association was reported between the prevalence of CPSP six months after surgery for breast cancer and 31 polymorphisms located across all seven genes. ${ }^{13}$ However, no correction for multiple testing was performed. The minor alleles of rs4766311 ( $p=$ $0.003), \mathrm{rs} 17376373(p=0.009), \mathrm{rs} 12995382(p=0.017)$ and $\mathrm{rs} 2542424(p=0.023)$ were associated with a lower prevalence of CPSP. For rs1072198 ( $p=0.048)$, rs17641121 ( $p=$ $0.011), r s 858003(p=0.03), r s 2835925(p=0.002), r s 2014712(p=0.041)$, and rs2545457 ( $p=0.022$ ) the minor allele was associated with a higher prevalence of CPSP. ${ }^{13}$ For the remaining polymorphisms, it was not mentioned whether the minor allele was associated with an increased or decreased prevalence of CPSP.

The association between two polymorphisms of the KCNS1 and the severity of CPSP gene was analysed in four postsurgical patient cohorts. ${ }^{30}$ The minor allele of rs13043825 was studied in two of these cohorts and was found to be associated with more severe CPSP after lumbar surgical discectomy ( $p=0.03)$, but not after limb amputation. The minor allele of $r 5734784$ was studied in all four cohorts and was associated with more severe CPSP after lumbar surgical discectomy ( $p=0.003$ ), and limb amputation ( $p=0.00012$ for phantom limb pain and $p=0.0033$ for stump pain), but not after mastectomy. ${ }^{30}$

\section{GCH1}

GCH1 encodes for the enzyme GTP cyclohydrolase 1, which is an important enzyme in the biosynthesis of catecholamines, serotonin and nitric oxide. ${ }^{31}$ Fourteen polymorphisms and two haplotypes of this gene were studied, of which only one was statistically significant before multiple testing correction. ${ }^{11}$ The minor allele of rs4411417 was associated with an increased prevalence of CPSP after several surgical procedures (OR 1.27, 95\% Cl 1.00-1.62, $p=0.046)$. Polymorphisms in the GCH1 gene 
were also investigated in two other studies, but these studies did not find a statistically significant association with the prevalence of CPSP. ${ }^{23,24}$

\section{CACNG2}

This gene encodes for the $\gamma-2$ transmembrane AMPA receptor regulatory protein stargazin, and is known for its role in cerebellar function and development of epilepsy. ${ }^{14}$ In only one study, the association between the CACNG2 gene and CPSP was investigated. Twelve polymorphisms and one haplotype of the CACNG2 gene were studied in a retrospective cohort of Israeli women after mastectomy or lumpectomy. ${ }^{14}$ The minor alleles of five of these polymorphisms (rs4820242, rs2284015, rs2284017, rs2284018, and rs1883988) were associated with an increased prevalence of CPSP. Three of the polymorphisms ( $r$ 4820242, rs2284015, rs2284017) were combined in a haplotype (containing nucleotides $\mathrm{A}-\mathrm{C}-\mathrm{C}$ respectively), that was associated with an increased prevalence of CPSP $(\mathrm{OR}=1.65, p=0.001)$.

\section{CHRNA6}

CHRNA6 codes for the a6 subunit of nicotinic acetylcholine receptors, which is expressed by dopamine-releasing neurons in the midbrain. ${ }^{32}$ Only one study investigated the CHRNA6 polymorphisms in relation to CPSP. ${ }^{12}$ Of the three polymorphisms, only one (rs7828365) was associated with CPSP. ${ }^{12}$ Patients carrying the minor allele showed an increased risk of developing CPSP six months after inguinal hernia repair (OR 12.0, $p=0.03$ ).

\section{$P 2 X 7 R$}

P2X7R codes for an ionotropic ATP-gated purinoceptors. ${ }^{33}$ This is a ligand-gated receptor that is located in the central and peripheral nervous system and immune cells. ${ }^{34}$ It is involved in mediating cell death, regulation of receptor trafficking, and inflammation. ${ }^{35}$ An association was reported between 23 polymorphisms in the $P 2 X 7 R$ gene and the severity of CPSP in patients after breast surgery for tumour removal and axillary lymph node dissection. ${ }^{36}$ The minor allele of rs208294 ( $\left.p=0.003\right)$ was associated with more severe CPSP and the minor alleles of rs7958311 ( $p=0.003)$ and rs208296 ( $p=0.006)$ were associated with less severe CPSP.

\section{Cytokines}

Cytokines are key messengers in the human body, and are important in the paracrine and autocrine signalling, and regulation of an inflammatory response. ${ }^{37}$ An association between 84 polymorphisms across 15 cytokine-affiliated genes and the prevalence of CPSP in women after surgery for breast cancer has been described. ${ }^{15}$ Thirteen of these polymorphisms showed a statistically significant association with the prevalence of 
severe CPSP (vs. no CPSP), but correction for multiple testing was not performed. The IFNG1 gene encodes for cytokine interferon $r$. Two polymorphisms in this gene; rs2069727 ( $p=0.025)$ and rs2069718 ( $p=0.006)$ were significantly associated with the prevalence of CPSP. The ILIRI and ILIR2 genes encode for interleukin receptors. Rs3917332 was significantly associated with CPSP ( $p=0.037)$, but it was not mentioned whether the minor allele was associated with an increased or decreased prevalence. The minor allele of rs11674595 was associated with an increased prevalence of CPSP (OR 36.0, 95\% Cl 2.02-643.37, $p=0.015$ ). Polymorphisms in interleukin genes were also reported to be associated with CPSP. ${ }^{15} \operatorname{Rs} 2243248$ ( $p=0.033$ ) in IL4, rs3024498 ( $p=$ $0.015), r s 1878672(p=0.029)$ and $r$ 3024491 $(p=0.035)$ in IL10 and rs1881457 ( $p=$ $0.043), \operatorname{rs} 1800925(p=0.007), r s 1295686(p=0.014)$ and $r s 20541(p=0.017)$ in IL13 were all associated with the prevalence of CPSP, but once again it was not mentioned whether the minor allele was associated with an increased or decreased prevalence. The NFKB1 encodes for the DNA binding subunit of nuclear factor-kB. ${ }^{38}$ The polymorphism rs4648141 was also reported to be associated with either an increased or decreased prevalence of CPSP. ${ }^{15}$ The NFKBIA gene encodes for an inhibitor of nuclear factor-kB transcription factor. ${ }^{39}$ The minor allele of the $r \$ 8904$ polymorphism was associated with an increased prevalence of CPSP (OR 1.21, 95\% Cl 1.01-1.44, $p=0.039$ ), but it was no longer statistically significant after multiple testing correction. ${ }^{11}$

\section{Human leukocyte antigens}

The human leukocyte antigens play an important role in the immune system. ${ }^{37}$ Twentythree polymorphisms and one haplotype were studied in relation to the prevalence of CPSP after inguinal hernia repair. ${ }^{40}$ Two polymorphisms; DRB1*04 (OR 2.28, 95\% Cl 1.32-3.96, $p=0.004$ ) and DQB1*03:02 (OR 3.16, 95\% Cl 1.61-6.22, $p=0.009$ ) were significantly more frequent in patients with CPSP. Both polymorphisms were combined into a haplotype, which was also associated with an increased prevalence of CPSP (OR $3.16,95 \% \mathrm{Cl}$ 1.61-6.22).

\section{DRD2}

The DRD2 gene encodes for the dopamine receptor. Dopamine is an important neurotransmitter involved in the transmission of nerve impulses, and is associated with many psychiatric disorders. ${ }^{41}$ Nine polymorphisms in the DRD2 gene were studied, two of which were associated with the prevalence of CPSP, but only before multiple testing correction. ${ }^{11}$ The minor alleles of rs4648317 (OR 1.35, 95\% Cl 1.05-1.74, $p=0.019$ ) and rs12364283 (OR 1.58, 95\% Cl 1.11-2.23, $p=0.0102$ ) were associated with a higher prevalence of CPSP. 


\section{ATXN1}

The ATXN1 encodes for the protein ataxin-1, which has been associated with neuronal death in spinocerebellar ataxia type $1 .{ }^{42}$ In the one study that analysed the ATXN1 gene, an association was reported between the minor allele of rs179997 and an increased prevalence of CPSP, but this was statistically significant only before multiple testing correction (OR 1.20, 95\% Cl 1.00-1.44, $p=0.0473){ }^{11}$

\section{Discussion}

In this systematic review, we summarize those studies that have focused on the association between genetic polymorphisms and the prevalence and severity of chronic postsurgical pain. Our results demonstrate that the overlap of the polymorphisms analysed between studies is minimal. Only a few polymorphisms within the COMT gene, the OPRM1 gene, potassium channel genes, GCH1, and the $I L 10$ gene, have been investigated by two or more studies. Most studies analysed a wide array of polymorphisms, and did not correct for multiple testing. ${ }^{11,13,15}$ From a methodological point of view, we therefore conclude that research on polymorphisms and the prevalence and severity of CPSP is still in its infancy. Nevertheless, in answer to our first research question, several genes might be associated with the prevalence and severity of CPSP, based on the studies included in this systematic review; COMT gene, OPRM1, potassium channel genes, GCH1, CACNG, CHRNA6, P2X7R, cytokine-associated genes, human leukocyte antigens, DRD2, and ATXN1.

In the context of pain, the COMT gene has been studied most extensively. The COMT gene has been shown to play an important role in experimental pain, nonsurgical chronic pain and even acute postoperative pain. ${ }^{26,43-47}$ COMT haplotypes which link the activity of the COMT enzyme to the sensitivity of pain, are still used today. ${ }^{26}$ Based on this systematic review the role of the COMT gene in CPSP is not fully clear; although a statistically significant association was reported, other studies were not able to confirm this finding. ${ }^{11,21-24}$ A recent study reported a significant association between COMT polymorphisms and pain-related functional impairment in the chronic postoperative phase. ${ }^{48}$ Strictly, this is not identical to CPSP, but these findings might suggest a role of COMT during the postoperative phase. A statistically significant association between a COMT diplotype and both pain and pain catastrophizing was noted. $^{25}$ Psychological factors, such as pain catastrophizing, surgical fear, and depression, have been shown to play an important role in the development of CPSP, and the COMT gene might be the common moderating factor. ${ }^{2,8,9}$ More research on the association between the COMT gene, pain and psychiatric disorders such as depression, anxiety and catastrophizing, might be of interest and might lead to preventive measure in the preoperative phase. 
The opioid receptor genes have also been extensively studied. Several studies have shown an association between polymorphisms within these genes and morphine use in the acute postoperative phase, especially for rs1799971 in the OPRM1 gene. ${ }^{49,50}$ This is not surprising, as polymorphisms in these genes might influence the expression of opioid receptors and reduce the affinity for exogenous opioids, and thus the magnitude of the expected analgesic effect. The role of opioid receptors in the chronic postoperative phase is more complex. Laboratory studies suggest that the receptor variant of the minor allele might be more sensitive to endogenous opioids (e.g. bendorphins), increasing the pain threshold, whereas the sensitivity to exogenous opioids is reduced. ${ }^{51}$ The rs1799971 polymorphism was found to be associated with an increased pain intensity in the chronic postoperative phase. ${ }^{22}$ This finding was not confirmed by two other studies. ${ }^{11,24}$ Although one of these studies was clearly underpowered to detect any effect, the other study included over a thousand patients and might have been powerful enough.

Potassium channels are also known to play an important role in nociceptive signalling, and polymorphisms in genes encoding for potassium channels are associated with different sorts of pain (e.g. postoperative pain, cancer pain, inflammatory pain, neuropathic pain). ${ }^{52,53}$ The KCNS1 gene is the most studied potassium channel gene in relation to CPSP. The minor allele of the rs 734784 polymorphism in this gene has been associated with more severe CPSP in several cohorts. ${ }^{13,}{ }^{30}$ Other potassium channel genes have been studied and associations with CPSP were reported. ${ }^{13}$ Even though more research is necessary, KCNS1 is an interesting target for future therapeutic strategies.

Polymorphisms in the GCH1 gene and the relation with CPSP was analysed in various studies. However, only one study reported a statistically significant association before multiple testing correction. ${ }^{11}$ Even though other studies have demonstrated a relationship between the $\mathrm{GCH} 1$ gene, and experimental and chronic non-surgical pain, more research will be necessary to determine whether an association between GCH1 and CPSP exists. ${ }^{31,54}$

Unfortunately, the included studies were not able to answer our second research question (Does adding the associated polymorphisms to existing prediction models improve their accuracy?) Even though almost all included studies corrected for one or several confounding factors, the confounders differed between the studies and none of the studies included all major known patient characteristic, clinical and psychological predictors. $1,2,55,56$ The results of this systematic review indicate that some polymorphisms might be interesting predictors for CPSP, but it would be interesting to know whether these polymorphisms can genuinely increase the reliability of the preexisting models. For instance, polymorphisms in the COMT gene were shown to be significantly associated with persistent post-herniotomy-related functional impairment, but when included into a general prediction model, the discriminatory power of the model did not significantly improve. ${ }^{48}$ Given that determination of polymorphisms on a 
per-patient basis is still relatively expensive, an increased discriminatory power of the prediction model is obviously needed before including genotyping into the analysis of preoperative patients.

A major limitation of this systematic review is the absence of a meta-analysis. After a careful analysis of the available literature, we decided not to perform a meta-analysis, because the studies were too heterogeneous, and too few per genetic variant. We noted a wide variety in the included surgical procedures, the instrument by which pain was measured and the follow-up time. Furthermore, various statistical tests were performed, which resulted in different ways of data presentation. The studies were also methodologically different. Most studies did not mention the use of multiple testing correction, and the presented $p$-values suggest that the results would no longer be statistically significant if multiple testing correction had been applied. Another research strategy might be more appropriate for the future.

Future research strategies might benefit from approaches used in other complex diseases, such as psychiatric disorders, where genetic vulnerability is characterized by many contributing polymorphisms, each with low effect size. By increasing the number of samples, genome-wide association studies (GWAS) have proved capable of reliably identification of common genetic risk variants for schizophrenia. ${ }^{57,58}$ Although the neurobiological effects of these variants are on the verge of being explored, this research has already yielded new biological hypothesis. In addition, polygenic risk scores, constructed based on explorative GWAS, successfully predict liability to schizophrenia, enabling researchers to estimate genetic liability at the individual level, and to use this information when studying for gene-environment interactions. ${ }^{59,60}$

Genome wide association studies of CPSP are still lacking. Only by performing these GWAS, can we identify accurately all the polymorphisms associated with the prevalence and severity of CPSP. Performing such studies would require the collection of large clinical samples, with clearly defined phenotypes. This can be achieved only through international consortia. ${ }^{10}$ It would certainly advance the knowledge on the genetic background of CPSP and other pain related phenotypes, and would create opportunities and targets for future hypothesis driven research.

In addition to this, as we explained before, we strongly suggest that future research should focus on incorporating genetic risk factors in general predictor models on the prevalence of CPSP.

In conclusion, many studies have investigated the association between several polymorphisms and the prevalence and severity of chronic postsurgical pain. The methodology of the included studies was too heterogeneous to enable accurate conclusions to be drawn. Another approach might be more appropriate in the future. Genome-wide association studies are still lacking in this field. Larger sample sizes, more replication efforts and alignment of primary outcome measures and confounding variables will be necessary. Based on this systematic review, promising genes include the COMT gene, opioid receptor genes, potassium channel genes, GCH1, CACNG, 
Chapter 5

CHRNA6, P2X7R, cytokine-associated genes, human leukocyte antigens, DRD2, and $A T X N 1$. Future research should be aimed at incorporating genetic risk factors with patient characteristic, clinical and psychological predictors for CPSP. 


\section{References}

1. Kehlet $\mathrm{H}$, Jensen TS, Woolf CJ. Persistent postsurgical pain: risk factors and prevention. Lancet 2006; 367(9522): 1618-25.

2. Hoofwijk DM, Fiddelers AA, Peters ML, et al. Prevalence and Predictive Factors of Chronic Postsurgical Pain and Poor Global Recovery One Year after Outpatient Surgery. Clin J Pain 2015.

3. Simanski CJ, Althaus A, Hoederath S, et al. Incidence of chronic postsurgical pain (CPSP) after general surgery. Pain Med 2014; 15(7): 1222-9.

4. Lavand'homme P. The progression from acute to chronic pain. Curr Opin Anaesthesiol 2011; 24(5): 54550.

5. Parsons B, Schaefer C, Mann R, et al. Economic and humanistic burden of post-trauma and post-surgical neuropathic pain among adults in the United States. J Pain Res 2013; 6: 459-69.

6. Peng Z, Li H, Zhang C, Qian X, Feng Z, Zhu S. A retrospective study of chronic post-surgical pain following thoracic surgery: prevalence, risk factors, incidence of neuropathic component, and impact on qualify of life. PloS one 2014; 9(2): e90014.

7. Macrae WA. Chronic post-surgical pain: 10 years on. Br J Anaesth 2008; 101(1): 77-86.

8. Theunissen HM, Peters ML, Bruce J, Gramke HF, Marcus MA. Preoperative Anxiety and Catastrophizing: A Systematic Review and Meta-analysis of the Association With Chronic Postsurgical Pain. Clin J Pain 2012; 28(9): 819-41.

9. Peters ML, Sommer M, de Rijke JM, et al. Somatic and psychologic predictors of long-term unfavorable outcome after surgical intervention. Ann Surg 2007; 245(3): 487-94.

10. Clarke H, Katz J, Flor H, Rietschel M, Diehl SR, Seltzer Z. Genetics of chronic post-surgical pain: a crucial step toward personal pain medicine. Can J Anaesth 2015; 62(3): 294-303.

11. Montes A, Roca G, Sabate S, et al. Genetic and Clinical Factors Associated with Chronic Postsurgical Pain after Hernia Repair, Hysterectomy, and Thoracotomy: A Two-year Multicenter Cohort Study. Anesthesiology 2015; 122(5): 1123-41.

12. Wieskopf JS, Mathur J, Limapichat W, et al. The nicotinic alpha6 subunit gene determines variability in chronic pain sensitivity via cross-inhibition of $\mathrm{P} 2 \times 2 / 3$ receptors. Science translational medicine 2015; 7(287): 287ra72.

13. Langford DJ, Paul SM, West CM, et al. Variations in potassium channel genes are associated with distinct trajectories of persistent breast pain after breast cancer surgery. Pain 2015; 156(3): 371-80.

14. Nissenbaum J, Devor M, Seltzer Z, et al. Susceptibility to chronic pain following nerve injury is genetically affected by CACNG2. Genome research 2010; 20(9): 1180-90.

15. Stephens K, Cooper BA, West C, et al. Associations between cytokine gene variations and severe persistent breast pain in women following breast cancer surgery. J Pain 2014; 15(2): 169-80.

16. Macrae WA. Chronic pain after surgery. Br J Anaesth 2001; 87(1): 88-98.

17. Joanna-Briggs-Institute. Joanna Briggs Institute Reviewers' Manual: 2008 Edition. 2008 ed. Adelaide: The Joanna Briggs Institute; 2008.

18. Downs SH, Black N. The feasibility of creating a checklist for the assessment of the methodological quality both of randomised and non-randomised studies of health care interventions. Journal of epidemiology and community health 1998; 52(6): 377-84.

19. Grossman MH, Emanuel BS, Budarf ML. Chromosomal mapping of the human catechol-Omethyltransferase gene to 22q11.1----q11.2. Genomics 1992; 12(4): 822-5.

20. Guldberg HC, Marsden CA. Catechol-O-methyl transferase: pharmacological aspects and physiological role. Pharmacological reviews 1975; 27(2): 135-206.

21. Rut M, Machoy-Mokrzynska A, Reclawowicz D, et al. Influence of variation in the catechol-Omethyltransferase gene on the clinical outcome after lumbar spine surgery for one-level symptomatic disc disease: a report on 176 cases. Acta neurochirurgica 2014; 156(2): 245-52.

22. Kolesnikov Y, Gabovits B, Levin A, et al. Chronic pain after lower abdominal surgery: do catechol-Omethyl transferase/opioid receptor mu-1 polymorphisms contribute? Molecular pain 2013; 9: 19. 
23. Hickey OT, Nugent NF, Burke SM, Hafeez P, Mudrakouski AL, Shorten GD. Persistent pain after mastectomy with reconstruction. Journal of clinical anesthesia 2011; 23(6): 482-8.

24. Lee PJ, Delaney P, Keogh J, Sleeman D, Shorten GD. Catecholamine-o-methyltransferase polymorphisms are associated with postoperative pain intensity. Clin J Pain 2011; 27(2): 93-101.

25. George SZ, Wallace MR, Wright TW, et al. Evidence for a biopsychosocial influence on shoulder pain: pain catastrophizing and catechol-O-methyltransferase (COMT) diplotype predict clinical pain ratings. Pain 2008; 136(1-2): 53-61.

26. Diatchenko L, Slade GD, Nackley AG, et al. Genetic basis for individual variations in pain perception and the development of a chronic pain condition. Human molecular genetics 2005; 14(1): 135-43.

27. Pasternak GW. Pharmacological mechanisms of opioid analgesics. Clinical neuropharmacology 1993; 16(1): 1-18.

28. Pan L, Xu J, Yu R, Xu MM, Pan YX, Pasternak GW. Identification and characterization of six new alternatively spliced variants of the human mu opioid receptor gene, Oprm. Neuroscience 2005; 133(1): 209-20.

29. Doyle DA, Morais Cabral J, Pfuetzner RA, et al. The structure of the potassium channel: molecular basis of K+ conduction and selectivity. Science 1998; 280(5360): 69-77.

30. Costigan M, Belfer I, Griffin RS, et al. Multiple chronic pain states are associated with a common amino acid-changing allele in KCNS1. Brain : a journal of neurology 2010; 133(9): 2519-27.

31. Tegeder I, Costigan M, Griffin RS, et al. GTP cyclohydrolase and tetrahydrobiopterin regulate pain sensitivity and persistence. Nature medicine 2006; 12(11): 1269-77.

32. Meyer EL, Yoshikami D, McIntosh JM. The neuronal nicotinic acetylcholine receptors alpha $4 *$ and alpha 6* differentially modulate dopamine release in mouse striatal slices. Journal of neurochemistry 2008; 105(5): 1761-9.

33. Rassendren F, Buell G, Newbolt A, North RA, Surprenant A. Identification of amino acid residues contributing to the pore of a P2X receptor. The EMBO journal 1997; 16(12): 3446-54.

34. Collo G, Neidhart S, Kawashima E, Kosco-Vilbois M, North RA, Buell G. Tissue distribution of the P2X7 receptor. Neuropharmacology 1997; 36(9): 1277-83.

35. Kawano A, Tsukimoto M, Mori D, et al. Regulation of P2X7-dependent inflammatory functions by P2X4 receptor in mouse macrophages. Biochemical and biophysical research communications 2012; 420(1): 102-7.

36. Sorge RE, Trang T, Dorfman R, et al. Genetically determined P2X7 receptor pore formation regulates variability in chronic pain sensitivity. Nature medicine 2012; 18(4): 595-9.

37. Abbas AK, A.H. L, S. P. Basic immunology: functions and disorders of the immune system: Elsevier Health Sciences; 2012.

38. Edenberg HJ, Xuei X, Wetherill LF, et al. Association of NFKB1, which encodes a subunit of the transcription factor NF-kappaB, with alcohol dependence. Human molecular genetics 2008; 17(7): 96370.

39. Verma IM, Stevenson JK, Schwarz EM, Van Antwerp D, Miyamoto S. Rel/NF-kappa B/I kappa B family: intimate tales of association and dissociation. Genes \& development 1995; 9(22): 2723-35.

40. Dominguez CA, Kalliomaki M, Gunnarsson U, et al. The DQB1 *03:02 HLA haplotype is associated with increased risk of chronic pain after inguinal hernia surgery and lumbar disc herniation. Pain 2013; 154(3): 427-33.

41. Meyer-Lindenberg A, Tost $\mathrm{H}$. Neural mechanisms of social risk for psychiatric disorders. Nature neuroscience 2012; 15(5): 663-8.

42. Rub U, Burk K, Timmann D, et al. Spinocerebellar ataxia type 1 (SCA1): new pathoanatomical and clinicopathological insights. Neuropathology and applied neurobiology 2012; 38(7): 665-80.

43. Diatchenko L, Nackley AG, Slade GD, et al. Catechol-O-methyltransferase gene polymorphisms are associated with multiple pain-evoking stimuli. Pain 2006; 125(3): 216-24.

44. Kambur O, Kaunisto MA, Tikkanen E, Leal SM, Ripatti S, Kalso EA. Effect of catechol-o-methyltransferasegene (COMT) variants on experimental and acute postoperative pain in 1,000 women undergoing surgery for breast cancer. Anesthesiology 2013; 119(6): 1422-33. 
45. Candiotti KA, Yang Z, Buric D, et al. Catechol-o-methyltransferase polymorphisms predict opioid consumption in postoperative pain. Anesth Analg 2014; 119(5): 1194-200.

46. Kolesnikov Y, Gabovits B, Levin A, Voiko E, Veske A. Combined catechol-O-methyltransferase and muopioid receptor gene polymorphisms affect morphine postoperative analgesia and central side effects. Anesth Analg 2011; 112(2): 448-53.

47. Tammimaki A, Mannisto PT. Catechol-O-methyltransferase gene polymorphism and chronic human pain: a systematic review and meta-analysis. Pharmacogenetics and genomics 2012; 22(9): 673-91.

48. Belfer I, Dai $F$, Kehlet $\mathrm{H}$, et al. Association of functional variations in COMT and GCH1 genes with postherniotomy pain and related impairment. Pain 2015; 156(2): 273-9.

49. Hwang IC, Park JY, Myung SK, Ahn HY, Fukuda K, Liao Q. OPRM1 A118G gene variant and postoperative opioid requirement: a systematic review and meta-analysis. Anesthesiology 2014; 121(4): 825-34.

50. Sia AT, Lim Y, Lim EC, et al. Influence of mu-opioid receptor variant on morphine use and self-rated pain following abdominal hysterectomy. J Pain 2013; 14(10): 1045-52.

51. Mura E, Govoni S, Racchi M, et al. Consequences of the $118 \mathrm{~A}>\mathrm{G}$ polymorphism in the OPRM1 gene: translation from bench to bedside? J Pain Res 2013; 6: 331-53.

52. Tsantoulas C. Emerging potassium channel targets for the treatment of pain. Current opinion in supportive and palliative care 2015; 9(2): 147-54.

53. Salinas M, Duprat F, Heurteaux C, Hugnot JP, Lazdunski M. New modulatory alpha subunits for mammalian Shab K+ channels. The Journal of biological chemistry 1997; 272(39): 24371-9.

54. Tegeder I, Adolph J, Schmidt H, Woolf CJ, Geisslinger G, Lotsch J. Reduced hyperalgesia in homozygous carriers of a GTP cyclohydrolase 1 haplotype. Eur J Pain 2008; 12(8): 1069-77.

55. Gramke HF, de Rijke JM, van Kleef $M$, et al. Predictive factors of postoperative pain after day-case surgery. Clin J Pain 2009; 25(6): 455-60.

56. Belfer I, Schreiber KL, Shaffer JR, et al. Persistent postmastectomy pain in breast cancer survivors: analysis of clinical, demographic, and psychosocial factors. J Pain 2013; 14(10): 1185-95.

57. Ripke S, O'Dushlaine C, Chambert K, et al. Genome-wide association analysis identifies 13 new risk loci for schizophrenia. Nature genetics 2013; 45(10): 1150-9.

58. Schizophrenia Working Group of the Psychiatric Genomics C. Biological insights from 108 schizophreniaassociated genetic loci. Nature 2014; 511(7510): 421-7.

59. Roussos P, Giakoumaki SG, Zouraraki C, et al. The Relationship of Common Risk Variants and Polygenic Risk for Schizophrenia to Sensorimotor Gating. Biological psychiatry 2015.

60. Iyegbe C, Campbell D, Butler A, Ajnakina O, Sham P. The emerging molecular architecture of schizophrenia, polygenic risk scores and the clinical implications for GxE research. Social psychiatry and psychiatric epidemiology 2014; 49(2): 169-82.

61. Sia AT, Sng BL, Lim EC, Law H, Tan EC. The influence of ATP-binding cassette sub-family B member -1 (ABCB1) genetic polymorphisms on acute and chronic pain after intrathecal morphine for caesarean section: a prospective cohort study. Int J Obstet Anesth 2010; 19(3): 254-60. 



\section{Chapter}

\section{Genetic polymorphisms and prediction of chronic postsurgical pain 3 and 12 months after hysterectomy}

Daisy M.N. Hoofwijk MD, Roel R.I. van Reij BSc, Bart P.F. Rutten MD PhD, Gunter Kenis PhD, Maurice Theunissen PhD, Elbert A. Joosten, PhD, Wolfgang F. Buhre MD PhD, Nynke J. van den Hoogen MSc 


\section{Abstract}

Chronic postsurgical pain (CPSP) is a serious problem. Clinical and psychological variables have not been able to explain all observed variance in prevalence and severity of CPSP. The first objective is to determine the association between genetic polymorphisms and the prevalence of CPSP after hysterectomy. The second objective is to analyze if the implementation of genetic polymorphisms into a previously performed clinical and psychological predictor analysis on the development of CPSP after hysterectomy will improve its discriminatory power. A prospective multicenter cohort study was performed in patients undergoing hysterectomy for benign indication. Clinical and psychological variables were collected by questionnaires in the week before surgery, postoperatively up to day 4, three and twelve months after hysterectomy. Blood was collected and 16 polymorphisms previously suggested to be correlated to CPSP (COMT, GCH1, KCNS1, CACNG2 and OPRM1) were genotyped. Logistic regression analyses were performed. A total of 345 patients were available for the genetic analyses. The prevalence of CPSP three months postoperatively was $10.5 \%$ and after twelve months $7.9 \%$. The polymorphism rs4818 within the COMT-gene was associated with the prevalence of CPSP after three months. No polymorphisms were associated with CPSP after twelve months. The addition of rs4818 to the prediction model did not change its discriminatory power significantly. 


\section{Introduction}

Chronic postsurgical pain (CPSP) is a common problem affecting up to $60 \%$ of surgical patients and is associated with negative outcomes such as a reduced quality of life and socio-economic consequences. ${ }^{1-6}$ Demographic, clinical and psychological risk factors for the development of CPSP have been well documented and include young age, procedure related risk factors, preexisting pain, acute postoperative pain, pain catastrophizing, surgical fear and lack of optimism. ${ }^{1,2,7-9}$ Unfortunately, these factors have not been able to explain all the observed variance in the prevalence and severity of CPSP. For this reason, the study and implementation of these polymorphisms have become of interest to further improve prediction models for CPSP. Data from twin studies and human pedigrees have estimated that the heritability of chronic pain generally ranges from $30-70 \% .{ }^{10}$ Especially polymorphisms in genes that encode for proteins involved in nerve conduction and transmission, opioid signaling and inflammatory processes have been suggested to play an important role in the development of CPSP. ${ }^{11-14}$ Identifying these genes could play an important role in finding targets for treatment.

Multiple studies have been performed that describe the association between genetic polymorphisms and the prevalence or severity of CPSP. ${ }^{11-17}$ Based on a systematic review an association between the prevalence or severity of CPSP and single nucleotide polymorphisms (SNPS) within the COMT (catechol-O-methyl transferase) gene, opioid receptor genes, potassium channel genes, GCH1 (GTP cyclohydrolase 1) gene, CACNG2 (calcium channel voltage gate dependent gamma subunit 2) gene, CHRNA6 (cholinergic receptor nicotinic alpha 6) gene, $P 2 X 7 R$ ( $22 X$ purinoceptor 7) gene, cytokine-associated genes, human leukocyte antigens, DRD2 (dopamine receptor D2) gene and ATXN1 (ataxin 1) gene was suggested. ${ }^{18}$ Nevertheless, most of these polymorphisms were researched by only one study, with the exception of polymorphisms within the COMT, OPRM1, GCH1, and KCNS1 genes. ${ }^{16,19-22}$ Furthermore, this systematic review revealed that several studies were of retrospective design, and most studies lacked statistical power and had only performed exploratory statistical analyses without correcting for multiple testing or known confounders. ${ }^{18}$ Interestingly, none of the studies used in the systematic review incorporated genetic polymorphisms in pre-existing prediction models for CPSP (i.e. based on demographic, clinical and psychological factors). As a result, it is unknown whether the inclusion of associated genetic polymorphisms will improve the discriminatory power of the existing clinical and psychological prediction models for the development of CPSP. It needs no further explanation that the latter is of significant clinical importance.

This study has two objectives. The first objective is to determine if genetic polymorphisms in the COMT, OPRM1, GCH1, CACNG2 and KCNS1 genes are associated with the prevalence of CPSP after hysterectomy. The second objective is to determine if the implementation of these genetic polymorphisms into a previously performed 
clinical and psychological predictor analysis for CPSP in this population will be able to improve its discriminatory power. ${ }^{23}$

\section{Methods}

\section{Study population}

The study was registered at the Dutch Trial Register under the number NTR2702 (http://www.trialregister.nl/trialreg/index.asp). An elaborate description of the study protocol has been published elsewhere. ${ }^{23}$ In short, a multicenter prospective cohort study was performed. Patients planned to undergo hysterectomy for a benign indication were included in one of 4 hospitals in the Netherlands: Maastricht University Medical Center+ (MUMC+) Maastricht, the Catharina Hospital (CzE) Eindhoven, the Máxima Medical Center (MMC) Veldhoven and the Orbis Medical Center (OMC) Sittard-Geleen. Inclusion took place between September 2010 and January 2014. Inclusion criteria were age between 18 and 65 years, good command of the Dutch language, elective surgery and total or subtotal hysterectomy with or without oophorectomy, and all types of surgical approach. Exclusion criteria were history of cancer, illiteracy and cognitive impairment. Patients who reported a malignancy or underwent another surgical procedure during the first postoperative year were also excluded. Approval of the local Medical Ethical Committee was obtained in all hospitals and all patients gave informed consent before participation.

\section{Collection of preoperative variables and predictors}

Patients were asked to fill out a baseline questionnaire before undergoing the hysterectomy. This questionnaire contained questions about demographics, (i.e. age, educational level, employment status, marital status, number of children, ethnicity), general health status and comorbidity, preoperative pain, and several psychological predictors. Preoperative pain was measured by using an adapted version of the Brief Pain Inventory, which determines the severity, location, duration and intensity of pain during the last week. ${ }^{24}$ An 11-item Numeric Rating Scale (NRS; where $0=$ no pain, and $10=$ worst pain imaginable) was used to measure pain intensity. Patients were asked to rate preoperative pain related to the planned hysterectomy and non-hysterectomy related pain separately. The Douleur Neuropathique 4 (DN4) was used to assess the neuropathic character of the pain. This instrument originally consists of 10 items, but only the 7 self-reported items were used (DN4-interview). Previous literature has demonstrated a sensitivity of $78 \%$ and specificity of $81 \%$ if only these 7 self-reported items are used. ${ }^{23,25}$ In agreement with previous literature a score of $\geq 3$ was defined as neuropathic pain. ${ }^{25}$ 
Psychological predictors included expected pain at the fourth postoperative day, surgical fear, pain catastrophizing, optimism, social support, depression, well-being and childhood abuse. Surgical fear was measured by the 8-item surgical fear questionnaire, of which four items measure short-term aspects of surgical fear and four items measure long-term aspects of fear. ${ }^{26}$ The 13-item Pain Catastrophizing Scale (PCS) was used to measure pain catastrophizing on three subscales; helplessness, rumination, and magnification. ${ }^{27}$ Optimism was assessed by using the 10 -item revised Life Orientation Test (LOT-R). ${ }^{28}$ Social support was measured by the 19-item Medical Outcomes Study social support survey (MOS-SSS), and by asking the patient for the number of close friends and relatives available for support. ${ }^{29}$ Depression was assessed with the Center for Epidemiological Studies - Depression (CES-D) questionnaire. ${ }^{30}$ Psychological wellbeing was assessed by the 12-item Well-Being Questionnaire (W-BQ12), which measures positive well-being, energy and negative well-being. ${ }^{31}$ To avoid overlap with the CES-D, the subscale of negative well-being was left out.

\section{Collection of perioperative variables and predictors}

Surgery-related and anesthesia-related variables were collected at the time of the procedure. These included type of incision, total or subtotal hysterectomy, with or without oophorectomy, indication for the hysterectomy, type of anesthesia, postoperative analgesic use, duration of the surgery, amount of blood loss, complications, and the amount of years of experience of the attending gynecologist.

Acute postoperative pain was assessed each day during the first four postoperative days. Patients were asked to rate their average and highest pain at rest and during movement, as well as the use of any analgesics. At the fourth postoperative day the DN4-interview was used to asses neuropathic pain.

\section{Collection of outcomes measures}

Questionnaires were used to measure pain severity at 3 and 12 months after hysterectomy. Patients were asked to rate hysterectomy related and non-hysterectomy related pain separately. Predictor analysis was performed with the highest hysterectomy related pain during the last week. In accordance with previous research an NRS $>3$ was defined as moderate to severe pain. $2,23,32,33$

\section{Genotyping and quality control}

Peripheral blood samples were obtained from the included patients. DNA was extracted from these samples at the Department of Clinical Genetics of the Maastricht University Medical Center+ (Maastricht, The Netherlands). Genotyping of SNPs within the following genes was performed at the Institute of Human Genetics at the UniKlinikum 
(Bonn, Germany) using the Infinium PsychArray v1.2 BeadChip (Illumina Inc., San Diego, CA, USA) and a customized Iplex array: COMT (rs4633, rs6269, rs4818, rs4680), GCH1 (rs10483639, rs3783641, rs8007267), KCNS1 (rs13043825, rs734784), CACNG2 (rs2284015, rs2284017, rs2284018, rs4820242, rs1883988) and OPRM1 (563649, rs1799971). These genes and polymorphisms were selected because they have previously been reported to be linked to CPSP. ${ }^{18}$

Genotypes were called using BeadStudio (Illumina Inc.). Quality control parameters included SNP callrate $<95 \%$, subject callrate $<95 \%$, deviation of the Hardy-Weinberg equilibrium $\left(p<1 \times 10^{-6}\right)$ and removal of rare variants with a minor allele frequency < 0.01 . After basic quality control heterozygosity was tested and outliers (> 3 standard deviations) were removed. Relatedness between the subjects was tested and one member of each pair was removed at random if relatedness was detected. All SNPs with $A<>T$ or $C<>G$ polymorphisms were removed from the set. After these control steps the SNPs were pruned to remove SNPs in $\operatorname{LD}\left(r^{2}>0.02\right)$. Principal components analysis was performed and the first two principal components were analyzed. The ancestry of the subjects was determined using HapMap, which showed that the outliers in the principal component analysis had a background other than Caucasian and were therefore removed from the sample.

Genotype imputation was performed using the imputation stepwise approach implemented in Minimac3 ${ }^{34}$ and Eagle v2.3 ${ }^{35}$. HRC r1.1 2016 panel was the reference panel, with population set to European. ${ }^{36}$ The output of the imputation was checked with the Will Rayner tool. Afterwards additional quality control was performed with genotype probability $>0.9$, quality threshold was set at 0.5 , INFO-score $>0.4$ and minor allele frequency and genotyping rate was checked again.

\section{Statistical analysis}

Patient characteristics and mean pain scores were evaluated using descriptive statistics. Before performing any other analyses, missing data were imputed by predictive mean matching. This method imputes values within the range of observed values in the data set, and is therefore robust to non-normal distribution of variables. The number of imputations was set to 5 . The results demonstrated are the pooled results based on these 5 datasets. In order to reduce the number of psychological predictors, a factor analysis with oblimin rotation was performed. Principal component analyses revealed 2 factors, which were named surgery-related worries (based on the SFQ and PCS) and general psychological robustness (based on the LOT-R, CES-D and W-BQ12). ${ }^{23}$

The association between the genetic polymorphisms and CPSP at 3 and 12 months was tested by univariate logistic regression analyses. An additive genetic model was tested, in which the number of minor alleles $(0,1$ or 2$)$ was defined as the candidate predictor. A $p$-value of $<0.05$ was considered statistically significant. 
In order to evaluate the effect of addition of genetic polymorphisms to a previously performed predictor analysis based on clinical and psychological variables, analyses were repeated using the same data. ${ }^{23}$ Separate analyses were performed for CPSP after 3 and 12 months. We first performed univariate logistic regression analyses on the same candidate predictors as were used in our previous publication (i.e. participating hospital, age, type of anesthesia, type of incision, paid job, educational level, American Society of Anesthesiology (ASA) physical status, number of pregnancies, surgery-related worries, general psychological robustness, history of sexual abuse, hysterectomy related baseline pain, non-hysterectomy related baseline pain, neuropathic pain at postoperative day 4 , training of attending gynecologist, surgery related infection during the first three postoperative months). For clinical and psychological predictors, the variable was only included in the multivariate logistic regression analysis if the univariate $p$-value was $<0.1$. Before including any of the genetic predictors in the regression model, correction for multiple testing was performed. This was done according to the method described by Galwey et. al., in which the effective number of tests is used instead of the actual number of tests. ${ }^{37,} 38$ In our study the effective number of tests was 11, which means that the SNP was only included in the regression model if the $p$-value in the univariate analysis was $<0.0091(0.1 / 11)$. After inclusion of all predictor variables in the regression model, a backward elimination analysis was performed until all remaining $p$-values were $<0.05$ for clinical and psychological variables or $<0.0045(0.05 / 11)$ for genetic variables.

For the variables hospital, age, type of anesthesia and type of incision a forced entry method was used in our previous publication, but because they were all non-significant, we did not use a forced entry in this analysis. After the final clinical and psychological prediction model was determined, the area under the curve (AUC) was calculated to investigate the model's discriminatory accuracy. After that, the genetic polymorphisms that were statistically significant in univariate logistic regression analyses were added to the multivariate prediction model, after which a backward elimination analysis was performed until all $p$-values were $<0.05$. The AUC was calculated for this final prediction model, and allows for determination whether the use of genetic analysis in clinical prediction models improves the chance of identifying patients at risk for CPSP after hysterectomy.

\section{Results}

\section{Flowchart and patient characteristics}

Figure 1 demonstrates the flowchart of inclusion and follow-up, which is an extension of the flowchart of our previous publication. ${ }^{23}$ Of the 517 patients that provided informed consent, 428 patients (82.8\%) provided baseline data and returned at least one of the 
follow-up questionnaires. Of these patients, 3 patients were excluded because no blood was available for genotyping and 80 patients were excluded because of insufficient quality of the isolated DNA, leaving 345 patients (80.6\%) with useful data for the genetic analyses. Of these 345 patients, 332 patients (96.2\%) returned the 3 month follow-up questionnaire, and 305 patients (91.9\%) returned the 12 month follow-up questionnaire.

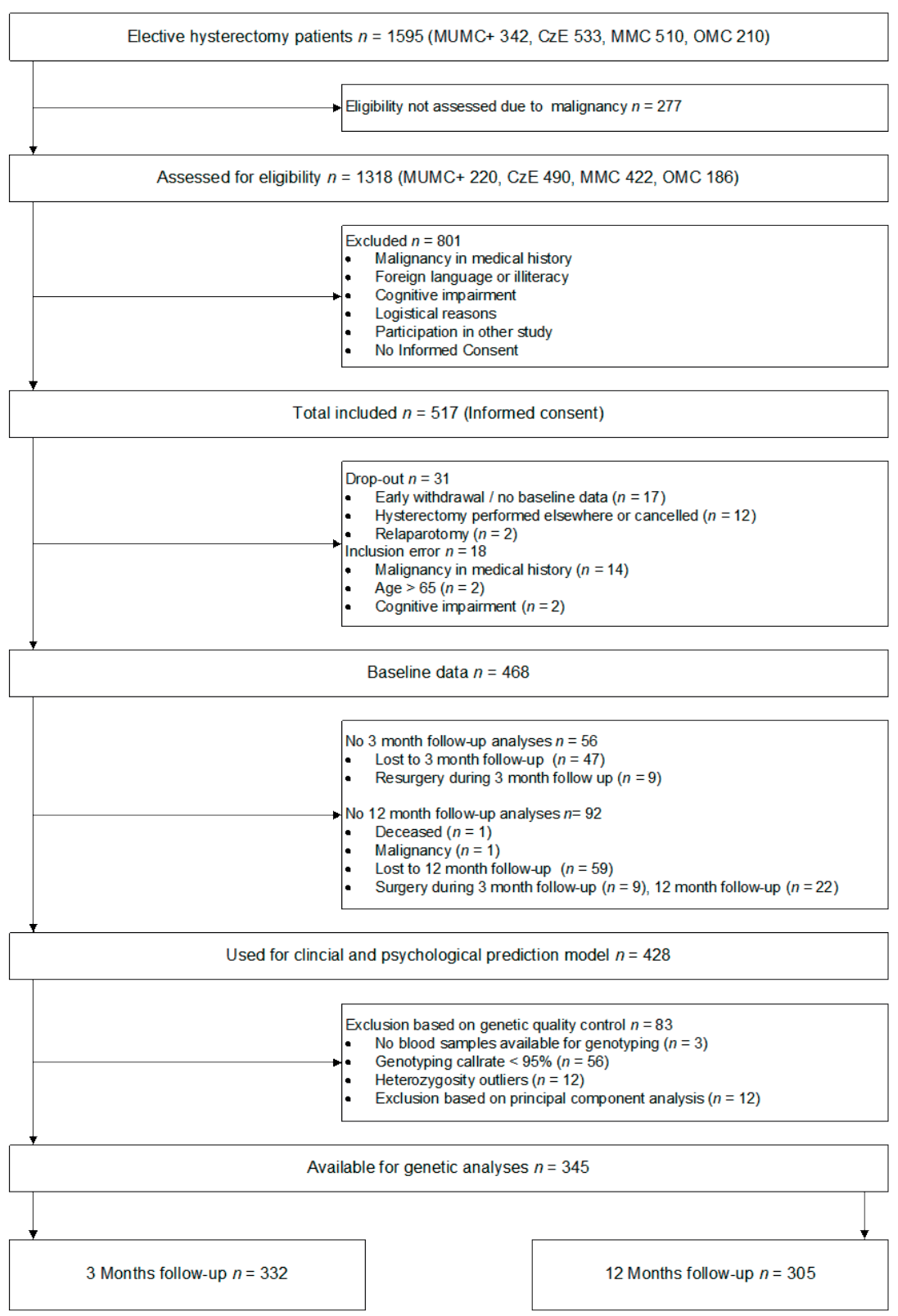

Figure 1. Flowchart of patient inclusion. 
Baseline patient characteristics are displayed in Table 1 and perioperative data are shown in Table 2. All patients included were females of European descent. The average age at the time of hysterectomy was 46,9 years (SD 7.2). The most frequently performed procedure was vaginal hysterectomy (65.8\%), and the most frequently performed anesthetic technique was general anesthesia (88.4\%). Most patients were ASA physical class I (54.8\%) or II (40.3\%). The prevalence of moderate to severe preoperative pain (NRS > 3) was rather high; $49.3 \%$ reported pain related to the planned hysterectomy and $34.2 \%$ reported non-hysterectomy related pain. Pain at the fourth postoperative day was also common; $62.5 \%$ of patients scored an NRS $>3$, and $20.8 \%$ of all patients scored $\geq 3$ at the DN4-interview.

Table 1. Baseline patient characteristics.

\begin{tabular}{|c|c|}
\hline & Baseline sample $(n=345)$ \\
\hline \multicolumn{2}{|l|}{ Center } \\
\hline Maastricht UMC+ & $116(33.6 \%)$ \\
\hline $\mathrm{CzE}$ & $106(30.7 \%)$ \\
\hline MMC & $87(25.2 \%)$ \\
\hline OMC & $36(10.4 \%)$ \\
\hline Age (mean, SD) & $46.9(7.2)$ \\
\hline \multicolumn{2}{|l|}{ Employment } \\
\hline Paid job & $252(73.3 \%)$ \\
\hline No paid job & $92(26.7 \%)$ \\
\hline \multicolumn{2}{|l|}{ Educational level } \\
\hline No/lower & $51(14.8 \%)$ \\
\hline Intermediate & $214(62.2 \%)$ \\
\hline University/higher & $79(23.0 \%)$ \\
\hline \multicolumn{2}{|l|}{ ASA physical class } \\
\hline 1 & $189(54.8 \%)$ \\
\hline II & $139(40.3 \%)$ \\
\hline III & $5(1.4 \%)$ \\
\hline \multicolumn{2}{|l|}{ Preoperative pain } \\
\hline Hysterectomy related pain (highest NRS > 3) & $170(49.3 \%)$ \\
\hline Non-hysterectomy related pain (highest NRS > 3) & $118(34.2 \%)$ \\
\hline \multicolumn{2}{|l|}{ History of sexual abuse } \\
\hline No & $272(79.5 \%)$ \\
\hline Yes & $70(20.5 \%)$ \\
\hline Number of pregnancies (mean, SD) & $2.5(1.2)$ \\
\hline \multicolumn{2}{|l|}{ Indication for hysterectomy (more than 1 possible) } \\
\hline Leiomyoma & $105(30.4 \%)$ \\
\hline Prolapse & $64(18.6 \%)$ \\
\hline Menorrhagia/metrorrhagia & $170(49.3 \%)$ \\
\hline Dysmenorrhoea & $22(6.4 \%)$ \\
\hline Endometriosis/adenomyosis & $18(5.2 \%)$ \\
\hline Abdominal pain & $18(5.2 \%)$ \\
\hline Cervial dysplasia & $20(5.8 \%)$ \\
\hline Other & $19(5.5 \%)$ \\
\hline
\end{tabular}

$\mathrm{ASA}=$ American Society of Anesthesiologist, Maastricht UMC+ = Maastricht University Medical Center+, CzE = Catharina Hospital Eindhoven, $\mathrm{MMC}=$ Máxima Medical Center Veldhoven, $\mathrm{OMC}=$ Orbis Medical Center Sittard-Geleen, NRS = Numeric Rating Scale, SD = Standard Deviation. 
Table 2. Perioperative data

\begin{tabular}{|c|c|}
\hline & Baseline sample $(n=345)$ \\
\hline \multicolumn{2}{|l|}{ Anesthesia type } \\
\hline General & $296(85.8 \%)$ \\
\hline Spinal & $40(11.6 \%)$ \\
\hline General \& epidural & $9(2.6 \%)$ \\
\hline \multicolumn{2}{|l|}{ Hysterectomy procedure } \\
\hline Total hysterectomy & $328(95.1 \%)$ \\
\hline Subtotal hysterectomy & $14(4.1 \%)$ \\
\hline \multicolumn{2}{|l|}{ Oophorectomy } \\
\hline No & $317(93 \%)$ \\
\hline Unilateral oophorectomy & $10(2.9 \%)$ \\
\hline Bilateral oophorectomy & $14(4.1 \%)$ \\
\hline \multicolumn{2}{|l|}{ Incision type } \\
\hline Total laparoscopic & $65(18.8 \%)$ \\
\hline Vaginal or laparoscopic assisted vaginal & $227(65.8 \%)$ \\
\hline Abdominal incision & $53(15.4 \%)$ \\
\hline Years of training of gynecologist (mean, SD) & $14.2(9.9)$ \\
\hline \multicolumn{2}{|l|}{ Pain at rest at PACU (1 hour after hysterectomy) } \\
\hline NRS $0-3$ & $168(54.5 \%)$ \\
\hline NRS 4-10 & $140(45.5 \%)$ \\
\hline \multicolumn{2}{|c|}{ Pain at postoperative day 4 (highest NRS last 24 hours) } \\
\hline NRS $0-3$ & $188(62.5 \%)$ \\
\hline NRS 4-10 & $113(37.5 \%)$ \\
\hline \multicolumn{2}{|l|}{ DN4 at day 4} \\
\hline $0-2$ & $228(79.2 \%)$ \\
\hline$\geq 3$ & $60(20.8 \%)$ \\
\hline
\end{tabular}

PACU = Post Anesthesia Care Unit, NRS = Numeric Rating Scale, SD = Standard Deviation, DN4 = Douleur Neuropathique 4

\section{Genetic polymorphisms and CPSP}

Three months after hysterectomy, 35 of 332 patients (10.5\%) experienced moderate to severe hysterectomy related pain (NRS $>3$ ). Twelve months after hysterectomy, the prevalence decreased to 24 of 305 patients (7.9\%).

Table 3 shows the location, minor alleles and minor allelic frequencies of all included SNPS. Almost all SNPs followed Hardy Weinberg equilibrium, with the exception of rs2284018. This is probably caused by the fact that the genotype TT was not present in our population. Therefore, the results of polymorphism rs2284018 need to be interpreted with caution. Table 4 demonstrates the results of the univariate regression analyses without correction for multiple testing. CPSP three months after hysterectomy was statistically significantly associated with the minor allele of rs4818 (OR [95\%Cl] 1.99 [1.19-3.33], $p$-value 0.009) and rs6269 (1.97 [1.18-3.28], $p$-value 0.01) within the COMTgene. After correction for multiple testing only rs4818 was eligible for inclusion in the prediction model for CPSP. All other tested SNPs, within the GCH1, CACNG, KCNS1 and $O P R M 1$ genes, were not significantly associated with the presence of moderate to severe 
hysterectomy related pain three months after the surgery. No association was found between the included SNPs and the severity of pain 12 months after hysterectomy.

Table 3. Description of included polymorphisms within our patient population

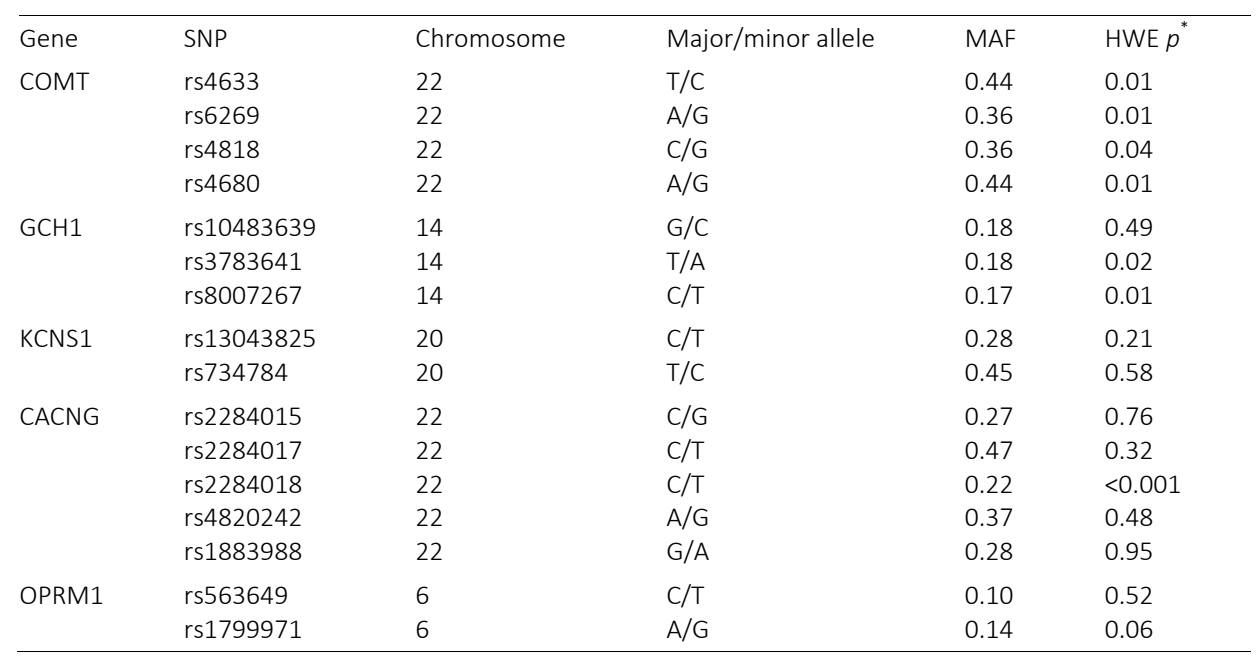

$\mathrm{SNP}=$ Single Nucleotide Polymorphism, MAF = Minor Allelic Frequency, HWE = Hardy Weinberg equilibrium.

Table 4. Association between included polymorphisms and chronic postsurgical pain (CPSP) at 3 and 12 months after hysterectomy

\begin{tabular}{|c|c|c|c|c|c|}
\hline \multirow[b]{2}{*}{ Gene } & \multirow[b]{2}{*}{ SNP } & \multirow{2}{*}{$\begin{array}{l}\text { CPSP at } 3 \text { months } \\
(n=332) \\
\text { OR }(95 \% \mathrm{Cl})\end{array}$} & \multirow[b]{2}{*}{$p$} & \multicolumn{2}{|l|}{$\begin{array}{l}\text { CPSP at } 12 \text { months } \\
(n=305)\end{array}$} \\
\hline & & & & OR $(95 \% \mathrm{Cl})$ & $p$ \\
\hline \multirow[t]{4}{*}{ COMT } & rs4633 & $1.59(0.95-2.66)$ & 0.08 & $1.09(0.59-2.02)$ & 0.78 \\
\hline & rs6269 & $1.97(1.19-3.26)$ & 0.01 & $1.22(0.67-2.24)$ & 0.52 \\
\hline & rs4818 & 1.99 (1.19-3.33) & 0.009 & $1.35(0.73-2.48)$ & 0.34 \\
\hline & rs4680 & $1.58(0.94-2.65)$ & 0.08 & $1.20(0.65-2.22)$ & 0.57 \\
\hline \multirow[t]{3}{*}{$\mathrm{GCH} 1$} & rs10483639 & $1.11(0.61-2.03)$ & 0.74 & $1.11(0.54-2.31)$ & 0.77 \\
\hline & rs3783641 & $1.04(0.57-1.90)$ & 0.90 & $1.13(0.56-2.30)$ & 0.73 \\
\hline & rs8007267 & $1.34(0.76-2.36)$ & 0.32 & $1.08(0.52-2.26)$ & 0.84 \\
\hline \multirow[t]{2}{*}{ KCNS1 } & rs13043825 & $0.86(0.48-1.53)$ & 0.61 & $0.91(0.46-1.77)$ & 0.77 \\
\hline & rs734784 & $0.77(0.47-1.29)$ & 0.32 & $0.85(0.47-1.55)$ & 0.60 \\
\hline \multirow[t]{5}{*}{ CACNG } & rs2284015 & $0.86(0.49-1.14)$ & 0.61 & $0.80(0.40-1.58)$ & 0.51 \\
\hline & rs2284017 & $1.15(0.90-1.47)$ & 0.57 & $0.89(0.50-1.60)$ & 0.70 \\
\hline & rs2284018 & $0.75(0.36-1.56)$ & 0.45 & $0.77(0.32-1.84)$ & 0.56 \\
\hline & rs4820242 & $1.35(0.81-2.26)$ & 0.25 & $1.19(0.65-2.18)$ & 0.57 \\
\hline & rs1883988 & $0.83(0.47-1.47)$ & 0.53 & $0.75(0.38-1.49)$ & 0.41 \\
\hline \multirow[t]{2}{*}{ OPRM1 } & rs563649 & $1.40(0.63-3.12)$ & 0.41 & $0.37(0.09-1.62)$ & 0.19 \\
\hline & rs1799971 & $1.25(0.60-2.60)$ & 0.56 & $0.54(0.18-1.62)$ & 0.27 \\
\hline
\end{tabular}

SNP = Single Nucleotide Polymorphism, OR = Odds ratio. 


\section{Discriminatory power of prediction models}

Because no SNPs were significantly associated with CPSP 12 months after hysterectomy in the univariate logistic regression analyses, we will only describe the prediction model for CPSP 3 months after hysterectomy. The following demographic, clinical and psychosocial variables were not associated with CPSP at 3 months in the univariate analyses and were therefore not used in the clinical prediction model: participating hospital, type of anesthesia, type of incision, educational level, ASA physical status, general psychological robustness, history of sexual abuse, and training of attending gynecologist.

The results of the logistic regression analysis for CPSP after three months are shown in Table 5. After performing the backward elimination analysis the following variables were included in the clinical prediction model: employment status, preoperative hysterectomy related pain, neuropathic pain at the fourth postoperative day, and selfreported infection during the first three postoperative months. The area under the receiver operating characteristic curve (AUC) for this clinical model was 0.78 .

In step 2, COMT rs4818 was added to the model. Rs4818 remained significant in the model, with an odds ratio of 2.50 (1.36-4.60). The addition of this variable increased the AUC of the prediction model from $0.78(95 \% \mathrm{Cl} 0.69-0.86)$ to $0.82(0,74-0,91)$, but this was not statistically significant.

Table 5. Prediction model for CPSP 3 months after hysterectomy, based on demographic, clinical, psychological and genetic factors

\begin{tabular}{|c|c|c|c|}
\hline Independent variable & OR $(95 \% \mathrm{Cl})$ & $P$-value & $A \cup C$ \\
\hline Step 1 & & & 0.78 \\
\hline \multicolumn{4}{|l|}{ Age } \\
\hline Per year & NS & $\mathrm{N} / \mathrm{A}$ & \\
\hline \multicolumn{4}{|l|}{ Employment } \\
\hline Paid job vs. no paid job & $0.39(0.17-0.91)$ & 0.03 & \\
\hline \multicolumn{4}{|l|}{ Pregnancies } \\
\hline Per number & NS & $N / A$ & \\
\hline \multicolumn{4}{|l|}{ Surgery-related worries } \\
\hline Per point (range $-1.68-2.60$ ) & NS & $\mathrm{N} / \mathrm{A}$ & \\
\hline \multicolumn{4}{|c|}{ Preoperative hysterectomy related pain } \\
\hline Per point on NRS (rang 0 - 10) & $1.18(1.04-1.35)$ & 0.01 & \\
\hline \multicolumn{4}{|c|}{ Preoperative non-hysterectomy related pain } \\
\hline Per point on NRS (rang $0-10$ ) & NS & $\mathrm{N} / \mathrm{A}$ & \\
\hline \multicolumn{4}{|c|}{ DN4-interview at postoperative day 4} \\
\hline$\geq 3$ vs. $0-2$ & $4.65(1.77-12.18)$ & 0.002 & \\
\hline \multicolumn{4}{|c|}{ Self-reported infection first 3 postoperative months } \\
\hline Yes vs. no & $6.81(2.66-17.45)$ & $<0.001$ & \\
\hline Step 2 & & & 0.82 \\
\hline \multicolumn{4}{|l|}{ rs4818 (COMT gene) } \\
\hline Per number of minor alleles & $2.50(1.36-4.60)$ & 0.003 & \\
\hline
\end{tabular}

NRS = Numeric Rating Scale, DN4 = Douleur Neuropathique 4, OR = Odds Ratio, Cl = Confidence Interval, AUC $=$ Area Under the Curve, NS = Not significant. 


\section{Discussion}

The first objective of this study was to determine if genetic polymorphisms in the COMT, OPRM1, GCH1, CACNG2 and KCNS1 genes are associated with the prevalence of CPSP after hysterectomy. This study shows that the rs4818 polymorphism within the COMT gene is associated with the presence of moderate to severe chronic postsurgical pain, three months after hysterectomy. The COMT gene encodes for the enzyme catechol-O-methyl transferase, which is essential in the breakdown of catecholamines. Polymorphisms within this gene can cause altered COMT enzyme activity. ${ }^{39,} 40$ The COMT gene has been studied extensively in relation to several pain syndromes. Associations have been found for experimental pain, for non-surgical chronic pain, for acute postoperative pain and for CPSP. ${ }^{41-46}$ In line with our findings, Rut et. al. reported a relationship between increasing severity of CPSP after lumbar discectomy and the minor allele (G) of rs4818, the minor allele (G) of rs4680, and homozygosity for the minor allele (T) of rs4633. ${ }^{16}$ On the other hand, in contrast to our findings, Rut and colleagues also described an association between less severe CPSP and the minor allele (G) of rs6269. Patients with the minor allele of rs6269 have been reported to experience more severe preoperative pain in their study. ${ }^{16}$ Two studies that investigated pain in the acute postoperative phase, demonstrated a relationship between increased pain severity and the presence of one or two minor alleles of rs4818 in patients after third molar extraction or surgery for breast cancer. ${ }^{21,43}$ An association between pain sensitivity in an experimental setting and several polymorphisms within the COMT gene in women with temperomandibular joint disorder has been reported, as patients with the major allele (A) of and the major allele (C) of rs4818 are more sensitive to pain. ${ }^{41}$ An association between several COMT polymorphisms and pain related functional impairment six months after inguinal hernia surgery has been described, as the presence of one or more minor alleles of rs4633 and rs6269 were associated with a protective effect. ${ }^{47}$

In sum, many studies reported associations between pain and polymorphisms within the COMT gene. However, the allele responsible for an increase in pain sensitivity or severity is not identical in all studies. Polymorphisms associated with increased pain severity in some studies were associated with a protective effect in other publications. ${ }^{16,21,41,43,46}$ Differences in types of pain (e.g. postoperative pain versus experimental pain and functional impairment) might be a possible explanation for these variations. Future studies are needed to further analyze the relation between types of pain, pain sensitivity or severity and polymorphisms in the COMT gene. Obviously it is very important to know the allele responsible for an increase in pain severity before these COMT polymorphisms can be included in general pain prediction models.

In this study, we were unable to demonstrate an association between the prevalence of CPSP after hysterectomy and polymorphisms within the GCH1, KCNS1, 
CACNG, and OPRM1 genes. Associations between CPSP and these polymorphism have been found in previous publications. ${ }^{11,13,14,17,18,22}$ It is very likely that our present study lacks statistical power to find an association between these polymorphisms and the presence of CPSP three and twelve months after hysterectomy. Future studies, in larger patient cohorts, are necessary to determine whether an association between these genes and CPSP exists.

The second objective of our study was to determine if the addition of genetic polymorphisms to the pre-existing clinical and psychological prediction model for CPSP after hysterectomy, would be able to improve the discriminatory power of the model. Our study demonstrates, for the first time, that the addition of polymorphisms within the COMT gene to the pre-existing prediction model improved the discriminatory power of the model from 'fair' (AUC 0.7-0.8) to 'good' (AUC 0.8-0.9). Even though the difference in AUC between a clinical prediction model and a prediction model with additional genetic factors was not statistically significance, we did show a trend towards improvement, and even small improvements in prediction value can be of clinical relevance in personalizing risk profiles and tailoring preventative strategies. ${ }^{47}$ Although many studies have found associations between polymorphisms in several genes and the prevalence and severity of CPSP, ${ }^{18}$ until now the additional value of using genetic polymorphisms in predicting CPSP has not been described. The additional value of using polymorphisms in pain related functional impairment after inguinal hernia repair has been reported, but pain related functional impairment is a different outcome than CPSP. ${ }^{47}$ Nevertheless, comparable findings between the two studies suggest that COMT polymorphisms play an important role in mediating pain processes during the chronic postoperative period. ${ }^{18,47}$ Future studies will be needed to determine whether polymorphisms within other genes might be able to even further improve clinical and psychological prediction models.

Many studies have shown that psychological predictors, such as pain catastrophizing, surgical fear and lack of optimism are associated with the prevalence and severity of CPSP, but we were not able to confirm these findings in the present study. $2,7,48$ For this study the considerable number of psychological predictors was reduced to two variables (i.e. general psychological robustness and surgery-related worries) based on a factor analysis. ${ }^{23}$ Combining multiple psychological predictors into a single variable might explain why we did not find a significant association between psychological robustness and CPSP. On the other hand, lack of statistical power might also be a reason for the absence of a association between psychological predictors and CPSP. However, there was to be enough power to detect an association between genetic polymorphisms and CPSP. Future studies are needed to determine the role of simultaneous use of psychological and genetic variables for the prediction of CPSP.

Important strengths of our study are both its prospective longitudinal design, as well as the homogeneity of the patient sample. All patients were of female gender, the same ethnicity and underwent the same surgery for benign indication. However, our study 
also has limitations. First of all, the sample size was relatively small for genetic research. The study was powered to perform a regression analysis with clinical and psychological variables. It is likely that larger sample sizes are necessary to detect the association between individual genetic polymorphisms and the prevalence of CPSP. In addition to this, the prevalence of CPSP was relatively low (only 35 patients after three months, and 24 patients after 12 months), which further decreases the statistical power. This could explain why we did find an association between polymorphisms and pain three months after hysterectomy, but not 12 months. The second limitation is that we only studied polymorphisms that have already been studied by other authors. No genome wide association studies (GWAS) have been performed yet with regard to CPSP.

Future studies on CPSP and genetics should focus on GWAS. This is the only way to identify all polymorphisms that can possibly be associated with the development of CPSP. This approach would require larger sample sizes and preferably homogeneous patient populations with a clearly defined pain phenotype. At the moment, our research group is working on a GWAS to determine if other polymorphisms are associated with CPSP after hysterectomy. After promising polymorphisms have been identified by GWAS, these polymorphisms can be tested and validated in large groups of patients, and various surgical procedures. We hypothesize that individual genes explain only a small part of the variation in pain phenotype, whereas a combination of multiple genes of interest might be able to explain a larger portion. This combination of polymorphisms can then be combined with clinical and psychological predictors to compose personalized risk profiles for standard procedures.

In conclusion, our study demonstrates that two polymorphisms within the COMT gene are associated with the prevalence of moderate to severe CPSP three months after hysterectomy. In line with recent literature, our results further indicate that polymorphisms within the COMT gene play an important role in postoperative mechanisms of pain processing. Addition of the rs4818 polymorphism to the prediction model showed a trend towards improvement. In order to determine the role of other genes on the development of CPSP, more research in larger patient cohorts is needed. 


\section{References}

1. Kehlet H, Jensen TS, Woolf CJ. Persistent postsurgical pain: risk factors and prevention. Lancet 2006; 367(9522): 1618-25.

2. Hoofwijk DM, Fiddelers AA, Peters ML, et al. Prevalence and Predictive Factors of Chronic Postsurgical Pain and Poor Global Recovery One Year after Outpatient Surgery. Clin J Pain 2015.

3. Simanski CJ, Althaus A, Hoederath $\mathrm{S}$, et al. Incidence of chronic postsurgical pain (CPSP) after general surgery. Pain Med 2014; 15(7): 1222-9.

4. Lavand'homme P. The progression from acute to chronic pain. Curr Opin Anaesthesiol 2011; 24(5): 54550.

5. Parsons B, Schaefer C, Mann R, et al. Economic and humanistic burden of post-trauma and post-surgical neuropathic pain among adults in the United States. J Pain Res 2013; 6: 459-69.

6. Peng Z, Li H, Zhang C, Qian X, Feng Z, Zhu S. A retrospective study of chronic post-surgical pain following thoracic surgery: prevalence, risk factors, incidence of neuropathic component, and impact on qualify of life. Plos one 2014; 9(2): e90014.

7. Macrae WA. Chronic post-surgical pain: 10 years on. Br J Anaesth 2008; 101(1): 77-86.

8. Katz J, Seltzer Z. Transition from acute to chronic postsurgical pain: risk factors and protective factors. Expert Rev Neurother 2009; 9(5): 723-44.

9. Peters ML, Sommer M, de Rijke JM, et al. Somatic and psychologic predictors of long-term unfavorable outcome after surgical intervention. Ann Surg 2007; 245(3): 487-94.

10. Clarke H, Katz J, Flor H, Rietschel M, Diehl SR, Seltzer Z. Genetics of chronic post-surgical pain: a crucial step toward personal pain medicine. Can J Anaesth 2015; 62(3): 294-303.

11. Montes A, Roca G, Sabate S, et al. Genetic and Clinical Factors Associated with Chronic Postsurgical Pain after Hernia Repair, Hysterectomy, and Thoracotomy: A Two-year Multicenter Cohort Study. Anesthesiology 2015; 122(5): 1123-41.

12. Wieskopf JS, Mathur J, Limapichat W, et al. The nicotinic alpha6 subunit gene determines variability in chronic pain sensitivity via cross-inhibition of P2X2/3 receptors. Science translational medicine 2015; 7(287): 287ra72.

13. Langford DJ, Paul SM, West CM, et al. Variations in potassium channel genes are associated with distinct trajectories of persistent breast pain after breast cancer surgery. Pain 2015; 156(3): 371-80.

14. Nissenbaum J, Devor M, Seltzer Z, et al. Susceptibility to chronic pain following nerve injury is genetically affected by CACNG2. Genome research 2010; 20(9): 1180-90.

15. Stephens K, Cooper BA, West C, et al. Associations between cytokine gene variations and severe persistent breast pain in women following breast cancer surgery. J Pain 2014; 15(2): 169-80.

16. Rut M, Machoy-Mokrzynska A, Reclawowicz D, et al. Influence of variation in the catechol-Omethyltransferase gene on the clinical outcome after lumbar spine surgery for one-level symptomatic disc disease: a report on 176 cases. Acta neurochirurgica 2014; 156(2): 245-52.

17. Kolesnikov Y, Gabovits B, Levin A, et al. Chronic pain after lower abdominal surgery: do catechol-Omethyl transferase/opioid receptor mu-1 polymorphisms contribute? Molecular pain 2013; 9: 19.

18. Hoofwijk DM, van Reij RR, Rutten BP, Kenis G, Buhre WF, Joosten EA. Genetic polymorphisms and their association with the prevalence and severity of chronic postsurgical pain: a systematic review. $\mathrm{Br} J$ Anaesth 2016; 117(6): 708-19.

19. George SZ, Wallace MR, Wright TW, et al. Evidence for a biopsychosocial influence on shoulder pain: pain catastrophizing and catechol-O-methyltransferase (COMT) diplotype predict clinical pain ratings. Pain 2008; 136(1-2): 53-61.

20. Kolesnikov Y, Gabovits B, Levin A, Voiko E, Veske A. Combined catechol-O-methyltransferase and muopioid receptor gene polymorphisms affect morphine postoperative analgesia and central side effects. Anesth Analg 2011; 112(2): 448-53.

21. Lee PJ, Delaney P, Keogh J, Sleeman D, Shorten GD. Catecholamine-o-methyltransferase polymorphisms are associated with postoperative pain intensity. Clin J Pain 2011; 27(2): 93-101. 
22. Costigan M, Belfer I, Griffin RS, et al. Multiple chronic pain states are associated with a common amino acid-changing allele in KCNS1. Brain : a journal of neurology 2010; 133(9): 2519-27.

23. Theunissen M, Peters ML, Schepers J, et al. Recovery 3 and 12 months after hysterectomy: epidemiology and predictors of chronic pain, physical functioning, and global surgical recovery. Medicine 2016; 95(26): e3980.

24. Cleeland CS, Ryan KM. Pain assessment: global use of the Brief Pain Inventory. Annals of the Academy of Medicine, Singapore 1994; 23(2): 129-38.

25. Bouhassira D, Attal N, Alchaar $\mathrm{H}$, et al. Comparison of pain syndromes associated with nervous or somatic lesions and development of a new neuropathic pain diagnostic questionnaire (DN4). Pain 2005; 114(1-2): 29-36.

26. Theunissen M, Peters ML, Schouten EG, et al. Validation of the surgical fear questionnaire in adult patients waiting for elective surgery. PloS one 2014; 9(6): e100225.

27. Sullivan MJ, Bishop SR. The Pain Catastrophizing Scale: Development and Validation. Psychological Assessment 1995; 7(4): 524-32.

28. Scheier MF, Carver CS, Bridges MW. Distinguishing optimism from neuroticism (and trait anxiety, selfmastery, and self-esteem): a reevaluation of the Life Orientation Test. J Pers Soc Psychol 1994; 67(6): 1063-78.

29. Sherbourne CD, Stewart AL. The MOS social support survey. Soc Sci Med 1991; 32(6): 705-14.

30. Radloff L. The CES-D scale: a self-report depression scale for research in the general population. App/ Psych Meas 1977; 1: 385-401.

31. Bradley C. The 12-Item Well-Being Questionnaire: origins, current stage of development, and availability. Diabetes care 2000; 23(6): 875.

32. Gerbershagen HJ, Rothaug J, Kalkman CJ, Meissner W. Determination of moderate-to-severe postoperative pain on the numeric rating scale: a cut-off point analysis applying four different methods. Br J Anaesth 2011; 107(4): 619-26.

33. Dihle A, Helseth S, Paul SM, Miaskowski C. The exploration of the establishment of cutpoints to categorize the severity of acute postoperative pain. Clin J Pain 2006; 22(7): 617-24.

34. Das S, Forer L, Schonherr S, et al. Next-generation genotype imputation service and methods. Nature genetics 2016; 48(10): 1284-7.

35. Loh PR, Danecek P, Palamara PF, et al. Reference-based phasing using the Haplotype Reference Consortium panel. Nature genetics 2016; 48(11): 1443-8.

36. McCarthy S, Das S, Kretzschmar W, et al. A reference panel of 64,976 haplotypes for genotype imputation. Nature genetics 2016; 48(10): 1279-83.

37. Galwey NW. A new measure of the effective number of tests, a practical tool for comparing families of non-independent significance tests. Genetic epidemiology 2009; 33(7): 559-68.

38. Li J, Ji L. Adjusting multiple testing in multilocus analyses using the eigenvalues of a correlation matrix. Heredity 2005; 95(3): 221-7.

39. Diatchenko L, Nackley AG, Slade GD, Fillingim RB, Maixner W. Idiopathic pain disorders--pathways of vulnerability. Pain 2006; 123(3): 226-30.

40. Nackley AG, Shabalina SA, Tchivileva IE, et al. Human catechol-O-methyltransferase haplotypes modulate protein expression by altering mRNA secondary structure. Science 2006; 314(5807): 1930-3.

41. Diatchenko L, Slade GD, Nackley AG, et al. Genetic basis for individual variations in pain perception and the development of a chronic pain condition. Human molecular genetics 2005; 14(1): 135-43.

42. Diatchenko L, Nackley AG, Slade GD, et al. Catechol-O-methyltransferase gene polymorphisms are associated with multiple pain-evoking stimuli. Pain 2006; 125(3): 216-24.

43. Kambur O, Kaunisto MA, Tikkanen E, Leal SM, Ripatti S, Kalso EA. Effect of catechol-o-methyltransferasegene (COMT) variants on experimental and acute postoperative pain in 1,000 women undergoing surgery for breast cancer. Anesthesiology 2013; 119(6): 1422-33.

44. Candiotti KA, Yang Z, Buric D, et al. Catechol-o-methyltransferase polymorphisms predict opioid consumption in postoperative pain. Anesth Analg 2014; 119(5): 1194-200. 


\section{Chapter 6}

45. Tammimaki A, Mannisto PT. Catechol-O-methyltransferase gene polymorphism and chronic human pain: a systematic review and meta-analysis. Pharmacogenetics and genomics 2012; 22(9): 673-91.

46. Belfer I, Segall S. COMT genetic variants and pain. Drugs of today 2011; 47(6): 457-67.

47. Belfer I, Dai $F$, Kehlet $H$, et al. Association of functional variations in COMT and GCH1 genes with postherniotomy pain and related impairment. Pain 2015; 156(2): 273-9.

48. Theunissen HM, Peters ML, Bruce J, Gramke HF, Marcus MA. Preoperative Anxiety and Catastrophizing: A Systematic Review and Meta-analysis of the Association With Chronic Postsurgical Pain. Clin J Pain 2012; 28(9): 819-41. 
Chapter

\section{General discussion}





\section{General discussion}

Every surgeon and anesthesiologist is aware of the fact that good perioperative patient care is unmistakably important to ensure optimal patient outcome. Outcome is often defined in terms of improvement of health status (e.g. curation of malignant disease, improvement of vascular status, etc) or improvement of functional status (e.g. return of joint mobility, improvement of vision), but outcome in terms of the patient's quality of life (QoL) should not be forgotten. Research has demonstrated that the presence of pain has a significant burden on quality of life, by affecting both physical and mental health status. ${ }^{1,2}$ Pain also has a significant influence on the patient's ability to participate in society, causes work impairment and increases use of health care resources. ${ }^{1,3,4}$

In order to ensure optimal patient outcome it is therefore paramount to make every possible effort to prevent the development of both acute and chronic postsurgical pain. A lot of literature is available with regard to acute postsurgical pain (APSP), and anesthesiologist are proficient at preventing and treating APSP by using multimodal analgesia techniques (e.g. combining analgesics, peripheral and neuraxial blocks, etc). ${ }^{5-7}$ Most hospitals have protocols in place to identify patients with APSP as early as possible, so that optimal treatment can be promptly initiated. ${ }^{8}$ However, with regard to the prevention and treatment of chronic postsurgical pain (CPSP), a lot is unknown. Therefore, protocols are often missing and thus optimal treatment is not performed. Nevertheless, the problem of CPSP is significant, as literature has already demonstrated that $10-50 \%$ of patients suffer from CPSP, and in 2-10\% of the cases CPSP is severe. 9,10 Surgeons and anesthesiologists need to be aware of this serious problem and methods of identifying patients at risk will be the first step towards prevention and treatment of CPSP.

This thesis aims to contribute to the knowledge regarding prevalence and risk factors of CPSP after several types of surgery. In the first part of this thesis we aim to identify demographic, clinical and psychological risk factors for the development of CPSP (Chapters II and III). These risk factors can be easily identified with use of questionnaires, and without invasive testing of patients. Furthermore, since clinical and psychological risk factors have only been able to explain part of the observed variance in prevalence and severity of CPSP, genetic factors have become of interest. It is therefore that in the second part of this thesis, we aim to identify genetic risk factors for the development of CPSP (Chapters V and VI). Moreover, the additional value of using genetic risk factors in clinical and psychological prediction models was determined (Chapter VI). In this general discussion the research questions as described in the general introduction (Chapter I) are answered and recommendations for future research are suggested. 


\section{$R Q$ 1: What is the prevalence of CPSP after relatively minor procedures in an outpatient setting?}

In order to answer this question we performed a large prospective cohort study of 908 patients undergoing a wide variety of outpatient procedures. Data were gathered by using three questionnaires. The first questionnaire was filled out a week before the surgery and included questions about demographic risk factors, present pain intensity and analgesic use, and three validated psychological questionnaires (i.e. Pain Catastrophizing Scale, Surgical Fear Questionnaire, Life Orientation Test). ${ }^{11-13}$ Four days after the surgery the second questionnaire was filled out to measure acute postoperative pain intensity and analgesic use. The main outcome parameter, CPSP, was measured one year after the outpatient surgery. Pain was measured by an 11-point numeric rating scale (NRS), and moderate to severe pain was defined as an NRS $>3 .{ }^{14,15}$ Patients were specifically asked to only rate the pain related to the surgical procedure.

The main outcome of this study was as follows: the prevalence of moderate to severe CPSP one year after outpatient surgery was $15.3 \%$, and in $3.2 \%$ of the cases the pain was rated as severe (NRS $>7$ ). A procedure-specific variation in the prevalence of CPSP was noted. In case of ophthalmologic surgery the prevalence of CPSP was only $2.9 \%$, but in case of orthopedic surgery $34.5 \%$ of patients reported moderate to severe CPSP.

Since we demonstrated that the prevalence of CPSP was extremely high after orthopedic surgery, and because arthroscopic knee surgery was one of the most commonly performed outpatient procedures in the Netherlands, ${ }^{16}$ a subgroup analyses was performed in 104 patients after outpatient knee arthroscopy. Partial meniscectomy was achieved in $77.9 \%$ of patients, in the remaining cases only debridement and lavage was performed. The prevalence of CPSP one year after arthroscopic knee surgery was $32.7 \%$. In $94.2 \%$ of the patients pain was the indication for the surgery, and fortunately $71.1 \%$ of the patients reported less pain after one year as compared to preoperatively, but $16.4 \%$ of patients reported more pain.

In conclusion, outpatient surgery was associated with a significant chance of developing CPSP in our cohort, but a procedure-specific variance was observed (Chapter II). Especially orthopedic surgery was associated with a high prevalence of CPSP (Chapter II \& III). In order to better understand the reasons behind this, we aimed to further the risk factors for the development of CPSP.

\section{RQ 2: Which demographic, clinical and psychological factors are associated with the development of CPSP after outpatient surgery?}

The same cohort of 908 patients undergoing outpatient surgery was used to answer the second research question. Studied demographic factors were age, gender, work status, and educational level. None of the demographic factors was associated with the 
presence of CPSP. Of the studied clinical variables preoperative pain, preoperative analgesic use, and acute postoperative pain (APSP) proved to be important predictors for the presence of moderate to severe CPSP. The predictive value of preoperative pain was the highest. An explanation can lie in the peripheral and central sensitization caused by pre-existing pain. ${ }^{9,} 10$ Preoperative pain has often been present during a longer period of time, while APSP is of a shorter nature. Preoperative pain might therefore by more likely to cause sensitization of the nervous system, and thus be a stronger predictor for CPSP. Other studies have confirmed the importance of both preoperative and acute postoperative pain as risk factors for the development of CPSP. ${ }^{10,17-20}$ The preoperative use of analgesics was also associated with CPSP in our cohort. In this case, the efficacy of analgesic use might in be more important, as we also demonstrated that patients with preoperative analgesics but without preoperative pain experienced less CPSP than patients with inadequately treated preoperative pain.

Tested psychological factors were surgical fear, optimism and pain catastrophizing. Both surgical fear and lack of optimism were associated with the development of CPSP in our cohort. Other studies have confirmed the importance of psychological factors with regard to the development of CPSP. ${ }^{20-22}$ Furthermore, poor preoperative quality of life also proved to be associated with CPSP in our cohort.

In conclusion, we were able to define preoperative pain, preoperative analgesic use, acute postoperative pain, surgical fear, lack of optimism and poor preoperative quality of life as risk factors for the development of CPSP (Chapter II). The association between preoperative pain and the presence of CPSP one year after outpatient surgery was the strongest.

\section{RQ 3: Is moderate to severe preoperative pain associated with an increase in postoperative hospital costs and health care utilization after outpatient surgery?}

A few small studies have shown that CPSP is associated with increased health care utilization. ${ }^{23,24}$ With regard to this economic burden, it would be interesting to know if it is possible to identify patients at risk for increased postoperative health care costs. Since we have already demonstrated that preoperative pain is one of the most important predictors for CPSP (Chapter II), ${ }^{25}{ }^{26}$ it is very important to know whether preoperative pain is also associated with increased postoperative health care costs.

In order to answer this question, we performed a large prospective cohort study in 1353 patients undergoing various types of outpatient surgery. Questionnaires were used to determine the severity of preoperative pain one week before the surgery. The amount of hospital-based health care costs during the first postoperative year was gathered from the hospital's data management system. Another questionnaire was filled out one year after the surgery, in which patients provided the number of visits to their general practitioner, medical specialist, emergency department or other health 
care professional. Patients were also asked about the number of days of absenteeism from their work or other social activities.

The main findings of this study were as follows: the average of hospital-based health care costs one year after outpatient surgery was $€ 1.520$. The average costs in patients with moderate to severe preoperative pain (€1.662) were statistically significantly higher than in patients without preoperative pain (€1.431). There was also a procedurespecific variance in postoperative costs. The highest costs were associated with ENTsurgery, dental surgery and orthopaedic surgery.

Patients with moderate to severe preoperative pain visited their general practitioner, medical specialist or other health care professional more often during first postoperative year than patients without preoperative pain. Patients with preoperative pain were more often absent from paid work during the first postoperative year as well.

In conclusion, in our cohort moderate to severe preoperative pain was associated with higher hospital-based health care costs during the first postoperative year, as well as an increased number of visits to the general practitioner, medical specialist or other health care professional and an increased amount of absenteeism from paid work (Chapter IV).

\section{RQ 4: Which genetic polymorphisms are associated with the prevalence and severity of CPSP?}

In order to answer this question we performed a systematic review of the available literature. We included 14 studies, describing a total of 5269 patients in 17 cohorts. The most commonly included surgical procedures were breast surgery ${ }^{27-32}$ and inguinal hernia repair. ${ }^{33-35}$ CPSP was measured at 3 months or more in all studies. The relatively small sample size and the large number of polymorphisms studied, resulted in low statistical power for most studies. Correction for multiple testing was reported by only one study. ${ }^{33}$ Furthermore, six of the included cohorts were of retrospective design. ${ }^{29-32}$ Both the heterogeneity in study design and the fact that only a few polymorphisms were reported by more than one study, precluded the performance of a meta-analysis.

Our systematic review demonstrated associations between the prevalence or severity of CPSP and polymorphisms within the following genes: the COMT (catechol-Omethyl transferase) gene, the OPRM1 (opioid receptor mu 1) gene, potassium channel genes, GCH1 (GTP cyclohydrolase 1) gene, CACNG2 (calcium channel voltage gate dependent gamma subunit 2) gene, CHRNA6 (cholinergic receptor nicotinic alpha 6) gene, P2X7R (P2X purinoceptor 7) gene, cytokine-associated genes, human leukocyte antigens, DRD2 (dopamine receptor D2) gene and ATXN1 (ataxin 1). It should be taken into account that only polymorphisms within COMT, OPRM1, GCH1 and KCNS1 were researched by more than one study.

Associations between CPSP and polymorphisms within the COMT gene were explored in several off the included studies, and two of them reported a significant 
association. $^{36,37}$ The minor alleles of rs4818 and rs4633 and the major alleles of rs6269 and rs4680 were correlated with an increased severity of CPSP. ${ }^{36}$ The importance of the COMT gene in pain processing mechanisms is confirmed by others studies, that have described associations between the COMT gene and experimental pain, non-surgical chronic pain and acute postoperative pain. ${ }^{38-42}$ Polymorphisms within the OPRM1 were investigated by three studies, but only one of these studies was able to confirm an association between the rs1799971 polymorphism and the severity of CPSP. ${ }^{43}$ Multiple studies have linked have previously linked the OPRM1 gene to morphine consumption in the acute postoperative phase. ${ }^{44,45}$ Of the potassium channel genes, the KCNS1 gene has been most extensively studied. The minor allele of rs 734784 has been linked to the severity of CPSP in several cohorts. ${ }^{27,31}$ Other polymorphisms within potassium channel genes have been described by only study, but without the correction for multiple testing. ${ }^{27}$ Polymorphisms of the $\mathrm{GCH} 1$ gene were researched in by studies, but only one found an association with the prevalence of CPSP, but only before multiple testing correction. ${ }^{33}$ Even though an association between $\mathrm{GCH} 1$ and both experimental pain and non-surgical chronic pain has been reported, ${ }^{46}$ more research is necessary to determine its role in the development of CPSP.

In conclusion, several studies have investigated the association between CPSP and genetic polymorphism, but the heterogeneity in methodology of these studies does not enable us to draw accurate conclusions. Nevertheless, promising genes can be selected and include the COMT gene, opioid receptor genes, potassium channel genes, GCH1, CACNG, CHRNA6, P2X7R, cytokine-associated genes, human leukocyte antigens, DRD2 and ATXN1.

\section{RQ 5 Does the addition of genetic risk factors to an existing clinical and demographic prediction model for CPSP after hysterectomy increase the accuracy of this model?}

Our systematic review (Chapter $\mathrm{V}$ ) demonstrates that several genetic polymorphisms are probably associated with the prevalence and severity of CPSP. ${ }^{47}$ However, none of the included studies researched the additional value of incorporating genetic polymorphisms into existing clinical and psychological prediction models for CPSP. Since determination of genetic polymorphisms requires a blood draw and is still relatively expensive on a per-patient basis, the additional value has to be demonstrated before it should be commonly deployed in a clinical setting.

In order to answer this research question we performed a large multicenter prospective cohort study in 345 patients undergoing hysterectomy for benign indication. Patients were asked to fill out several questionnaires. ${ }^{22}$ The baseline questionnaire before the hysterectomy included questions about demographics, general health status, preoperative pain and several psychological predictors. Surgeryrelated and anesthesia-related variables were collected at the time of the procedure. 
Acute postoperative pain was assessed by questionnaire each day during the first four postoperative days. At three and twelve months after hysterectomy patients were asked to fill out questionnaires with regard to CPSP. Peripheral blood samples for genotyping were obtained at the time of the surgery. Polymorphisms selected for genotyping were within the COMT gene, GCH1, KCNS1 gene, OPRM1 gene and CACNG2 gene. These genes were selected because they have been previously linked to CPSP (Chapter V). ${ }^{47}$

Clinical and psychological risk factors for CPSP in this cohort were shown to be hysterectomy-related and nonhysterectomy-related preoperative pain, number of pregnancies, surgery-related worries, neuropathic pain at day 4, and self-reported surgery-related infection at 3 months. ${ }^{22}$ In our analysis for genetic risk factors in the very same patient population, only rs 4818 was associated with the prevalence of CPSP three months after hysterectomy, and no polymorphisms were associated with CPSP after twelve months. The area under the curve (AUC) of the prediction model improved from 0.78 to 0.82 with the addition of the $\mathrm{rs} 4818$ polymorphism, but this was however not statistically significant. A possible explanation could be the relatively small size of the study population. This research should be repeated in larger patient cohorts.

In conclusion, the rs4818 polymorphisms within the COMT-gene was associated with the prevalence of CPSP three months after hysterectomy, but the implementation of rs4818 into the clinical and psychological prediction model, did not significantly improve the chance of identifying hysterectomy patients at risk for CPSP.

\section{Recommendations for future research}

In order to further clarify the prevalence and risk factors of CPSP, future research should not only focus on the intensity of the reported pain (e.g. NRS or VAS), but also on the characteristics (e.g. neuropathic, inflammatory, etc.) and the location of the reported pain. Not only is this necessary in order to discriminate between the continuation of a pre-existing problem and newly formed postoperative pain, ${ }^{48}$ as it is known that various types of pain do have a different origin and are therefore associated with different risk profiles. Moreover, pain should not be the only outcome measure studied. Other Patient Reported Outcome Measures (PROMs, e.g. postoperative quality of life, quality of recovery, etc.) could give a more general impression of the success of the procedure from the patient's point of view. ${ }^{49-51}$ This is especially true for procedures that are performed in order to improve the patient's quality of life.

The identification of genetic risk factors for the development of CPSP is still in its infancy. Most studies have so far focused on genetic polymorphisms that have been associated with other types of pain (hypotheses-driven research). A different approach, in the form of genome-wide association studies (GWAS) is necessary in order to identify promising polymorphisms in CPSP. With GWAS, an extremely large amount (millions) of polymorphisms spread over the entire genome is studied simultaneously. ${ }^{52}$ This method 
can help formulate hypotheses as to which polymorphisms are likely to be correlated with CPSP (hypotheses-generating research). ${ }^{53}$ Once GWAS have identified promising polymorphisms in multiple large patient cohorts, these polymorphisms can be tested in smaller procedure-specific cohorts and incorporated into clinical and psychological prediction models.

All together, prediction and prevention of CPSP needs further research. Future studies will have to focus on interventions to reduce the prevalence and severity of CPSP. Small studies have already shown that the prevalence of CPSP is decreased with treatment of acute postoperative wound pain and neuropathic pain. ${ }^{54,55}$ However, since our studies demonstrate that preoperative pain appears to be the strongest predictor for the development of CPSP, it would be interesting to know if optimal treatment of preoperative pain would be able to reduce the prevalence and severity of CPSP. Furthermore, the role of targeting preoperative anxiety, pain catastrophizing and pessimism in the prevention of CPSP, should also be clarified. More research in the field of genetics and CPSP will further improve prediction models, but might also lead to new targets for prevention and treatment of CPSP. 


\section{References}

1. Langley P, Muller-Schwefe G, Nicolaou A, Liedgens H, Pergolizzi J, Varrassi G. The societal impact of pain in the European Union: health-related quality of life and healthcare resource utilization. Journal of medical economics 2010; 13(3): 571-81.

2. Goren A, Mould-Quevedo J, daCosta DiBonaventura M. Prevalence of pain reporting and associated health outcomes across emerging markets and developed countries. Pain Med 2014; 15(11): 1880-91.

3. Langley P, Perez Hernandez C, Margarit Ferri C, Ruiz Hidalgo D, Lubian Lopez M. Pain, health related quality of life and healthcare resource utilization in Spain. Journal of medical economics 2011; 14(5): 62838.

4. Langley PC. The societal burden of pain in Germany: health-related quality-of-life, health status and direct medical costs. Journal of medical economics 2012; 15(6): 1201-15.

5. Wardhan R, Chelly J. Recent advances in acute pain management: understanding the mechanisms of acute pain, the prescription of opioids, and the role of multimodal pain therapy. F1000Research 2017; 6: 2065.

6. Buvanendran A, Kroin JS. Multimodal analgesia for controlling acute postoperative pain. Curr Opin Anaesthesiol 2009; 22(5): 588-93.

7. Elvir-Lazo OL, White PF. The role of multimodal analgesia in pain management after ambulatory surgery. Curr Opin Anaesthesiol 2010; 23(6): 697-703.

8. Inspectie voor de gezondheidszorg I. Basisset Medisch Specialistische Zorg Kwaliteitsindicatoren. 2017. https://http://www.igj.nl/documenten/indicatorensets/2016/08/08/basisset-medisch-specialistischezorg-2017.

9. Kehlet $\mathrm{H}$, Jensen TS, Woolf CJ. Persistent postsurgical pain: risk factors and prevention. Lancet 2006; 367(9522): 1618-25.

10. Fletcher D, Stamer UM, Pogatzki-Zahn E, et al. Chronic postsurgical pain in Europe: An observational study. Eur J Anaesthesiol 2015; 32(10): 725-34.

11. Sullivan MJ, Bishop SR. The Pain Catastrophizing Scale: Development and Validation. Psychological Assessment 1995; 7(4): 524-32.

12. Theunissen $M$, Peters ML, Schouten EG, et al. Validation of the surgical fear questionnaire in adult patients waiting for elective surgery. PloS one 2014; 9(6): e100225.

13. Scheier MF, Carver CS, Bridges MW. Distinguishing optimism from neuroticism (and trait anxiety, selfmastery, and self-esteem): a reevaluation of the Life Orientation Test. J Pers Soc Psychol 1994; 67(6): 1063-78.

14. Gerbershagen HJ, Rothaug J, Kalkman CJ, Meissner W. Determination of moderate-to-severe postoperative pain on the numeric rating scale: a cut-off point analysis applying four different methods. Br J Anaesth 2011; 107(4): 619-26.

15. Dihle A, Helseth S, Paul SM, Miaskowski C. The exploration of the establishment of cutpoints to categorize the severity of acute postoperative pain. Clin J Pain 2006; 22(7): 617-24.

16. Operaties in het ziekenhuis; soort opname, leeftijd en geslacht, 1995-2010. 05-02-2014. http://statline.cbs.nl/Statweb/publication/?DM=SLNL\&PA=80386NED\&D1=a\&D2=0\&D3=0\&D4=a\&D5=1 \&HDR=T\&STB=G4\%2cG1\%2cG2\%2cG3\&VW=T.

17. Macrae WA. Chronic pain after surgery. Br J Anaesth 2001; 87(1): 88-98.

18. Aasvang EK, Gmaehle E, Hansen JB, et al. Predictive risk factors for persistent postherniotomy pain. Anesthesiology 2010; 112(4): 957-69.

19. Katz J, Seltzer Z. Transition from acute to chronic postsurgical pain: risk factors and protective factors. Expert Rev Neurother 2009; 9(5): 723-44.

20. Peters ML, Sommer M, de Rijke JM, et al. Somatic and psychologic predictors of long-term unfavorable outcome after surgical intervention. Ann Surg 2007; 245(3): 487-94. 
21. Theunissen HM, Peters ML, Bruce J, Gramke HF, Marcus MA. Preoperative Anxiety and Catastrophizing: A Systematic Review and Meta-analysis of the Association With Chronic Postsurgical Pain. Clin J Pain 2012; 28(9): 819-41.

22. Theunissen M, Peters ML, Schepers J, et al. Recovery 3 and 12 months after hysterectomy: epidemiology and predictors of chronic pain, physical functioning, and global surgical recovery. Medicine 2016; 95(26): e3980.

23. Parsons B, Schaefer C, Mann R, et al. Economic and humanistic burden of post-trauma and post-surgical neuropathic pain among adults in the United States. J Pain Res 2013; 6: 459-69.

24. Hollisaaz MT, Noorbala MH, Irani N, et al. Severity of chronic pain affects health care utilization after kidney transplantation. Transplant Proc 2007; 39(4): 1122-5.

25. Hoofwijk DM, Fiddelers AA, Emans PJ, et al. Prevalence and Predictive Factors of Chronic Postsurgical Pain and Global Surgical Recovery 1 Year After Outpatient Knee Arthroscopy: A Prospective Cohort Study. Medicine 2015; 94(45): e2017.

26. Hoofwijk DM, Fiddelers AA, Peters ML, et al. Prevalence and Predictive Factors of Chronic Postsurgical Pain and Poor Global Recovery One Year after Outpatient Surgery. Clin J Pain 2015.

27. Langford DJ, Paul SM, West CM, et al. Variations in potassium channel genes are associated with distinct trajectories of persistent breast pain after breast cancer surgery. Pain 2015; 156(3): 371-80.

28. Stephens K, Cooper BA, West C, et al. Associations between cytokine gene variations and severe persistent breast pain in women following breast cancer surgery. J Pain 2014; 15(2): 169-80.

29. Sorge RE, Trang T, Dorfman R, et al. Genetically determined P2X7 receptor pore formation regulates variability in chronic pain sensitivity. Nature medicine 2012; 18(4): 595-9.

30. Hickey OT, Nugent NF, Burke SM, Hafeez P, Mudrakouski AL, Shorten GD. Persistent pain after mastectomy with reconstruction. Journal of clinical anesthesia 2011; 23(6): 482-8.

31. Costigan M, Belfer I, Griffin RS, et al. Multiple chronic pain states are associated with a common amino acid-changing allele in KCNS1. Brain : a journal of neurology 2010; 133(9): 2519-27.

32. Nissenbaum J, Devor M, Seltzer Z, et al. Susceptibility to chronic pain following nerve injury is genetically affected by CACNG2. Genome research 2010; 20(9): 1180-90.

33. Montes A, Roca G, Sabate S, et al. Genetic and Clinical Factors Associated with Chronic Postsurgical Pain after Hernia Repair, Hysterectomy, and Thoracotomy: A Two-year Multicenter Cohort Study. Anesthesiology 2015; 122(5): 1123-41.

34. Wieskopf JS, Mathur J, Limapichat $\mathrm{W}$, et al. The nicotinic alpha6 subunit gene determines variability in chronic pain sensitivity via cross-inhibition of $\mathrm{P} 2 \times 2 / 3$ receptors. Science translational medicine 2015; 7(287): 287ra72.

35. Dominguez CA, Kalliomaki M, Gunnarsson U, et al. The DQB1 *03:02 HLA haplotype is associated with increased risk of chronic pain after inguinal hernia surgery and lumbar disc herniation. Pain 2013; 154(3): 427-33.

36. Rut M, Machoy-Mokrzynska A, Reclawowicz D, et al. Influence of variation in the catechol-Omethyltransferase gene on the clinical outcome after lumbar spine surgery for one-level symptomatic disc disease: a report on 176 cases. Acta neurochirurgica 2014; 156(2): 245-52.

37. George SZ, Wallace MR, Wright TW, et al. Evidence for a biopsychosocial influence on shoulder pain: pain catastrophizing and catechol-O-methyltransferase (COMT) diplotype predict clinical pain ratings. Pain 2008; 136(1-2): 53-61.

38. Diatchenko L, Slade GD, Nackley AG, et al. Genetic basis for individual variations in pain perception and the development of a chronic pain condition. Human molecular genetics 2005; 14(1): 135-43.

39. Diatchenko L, Nackley AG, Slade GD, et al. Catechol-O-methyltransferase gene polymorphisms are associated with multiple pain-evoking stimuli. Pain 2006; 125(3): 216-24.

40. Kambur O, Kaunisto MA, Tikkanen E, Leal SM, Ripatti S, Kalso EA. Effect of catechol-o-methyltransferasegene (COMT) variants on experimental and acute postoperative pain in 1,000 women undergoing surgery for breast cancer. Anesthesiology 2013; 119(6): 1422-33.

41. Candiotti KA, Yang Z, Buric D, et al. Catechol-o-methyltransferase polymorphisms predict opioid consumption in postoperative pain. Anesth Analg 2014; 119(5): 1194-200. 
42. Kolesnikov Y, Gabovits B, Levin A, Voiko E, Veske A. Combined catechol-O-methyltransferase and muopioid receptor gene polymorphisms affect morphine postoperative analgesia and central side effects. Anesth Analg 2011; 112(2): 448-53.

43. Kolesnikov Y, Gabovits B, Levin A, et al. Chronic pain after lower abdominal surgery: do catechol-Omethyl transferase/opioid receptor mu-1 polymorphisms contribute? Molecular pain 2013; 9: 19.

44. Hwang IC, Park JY, Myung SK, Ahn HY, Fukuda K, Liao Q. OPRM1 A118G gene variant and postoperative opioid requirement: a systematic review and meta-analysis. Anesthesiology 2014; 121(4): 825-34.

45. Sia AT, Lim Y, Lim EC, et al. Influence of mu-opioid receptor variant on morphine use and self-rated pain following abdominal hysterectomy. J Pain 2013; 14(10): 1045-52.

46. Tegeder I, Costigan M, Griffin RS, et al. GTP cyclohydrolase and tetrahydrobiopterin regulate pain sensitivity and persistence. Nature medicine 2006; 12(11): 1269-77.

47. Hoofwijk DM, van Reij RR, Rutten BP, Kenis G, Buhre WF, Joosten EA. Genetic polymorphisms and their association with the prevalence and severity of chronic postsurgical pain: a systematic review. $\mathrm{Br} J$ Anaesth 2016; 117(6): 708-19.

48. Werner MU, Kongsgaard UE. I. Defining persistent post-surgical pain: is an update required? Br J Anaesth 2014; 113(1): 1-4.

49. Stessel B, Fiddelers AA, Joosten EA, Hoofwijk DM, Gramke HF, Buhre WF. Prevalence and Predictors of Quality of Recovery at Home After Day Surgery. Medicine 2015; 94(39): e1553.

50. Wong J, Tong D, De Silva Y, Abrishami A, Chung F. Development of the functional recovery index for ambulatory surgery and anesthesia. Anesthesiology 2009; 110(3): 596-602.

51. Jakobsson J. Assessing recovery after ambulatory anaesthesia, measures of resumption of activities of daily living. Curr Opin Anaesthesiol 2011; 24(6): 601-4.

52. Kraft P, Cox DG. Study designs for genome-wide association studies. Advances in genetics 2008; 60: 465504.

53. Geschwind DH, Flint J. Genetics and genomics of psychiatric disease. Science 2015; 349(6255): $1489-94$.

54. Bouman EA, Theunissen M, Bons SA, et al. Reduced incidence of chronic postsurgical pain after epidural analgesia for abdominal surgery. Pain practice : the official journal of World Institute of Pain 2014; 14(2): E76-84.

55. Clarke H, Bonin RP, Orser BA, Englesakis M, Wijeysundera DN, Katz J. The prevention of chronic postsurgical pain using gabapentin and pregabalin: a combined systematic review and meta-analysis. Anesth Analg 2012; 115(2): 428-42. 
Chapter

\section{Summary}

Samenvatting 



\section{Summary}

Chronic postsurgical pain (CPSP) remains a frequent problem with serious consequences, such as a reduced quality of life, absenteeism from work and social activities and an increase in health care consumption. The aim of this thesis was improve our insight into the complex interaction of clinical, psychological and genetic risk factors for the development of CPSP after a wide variety of surgical procedures.

The results of a large prospective cohort study, describing the prevalence and predictors of CPSP in patients undergoing a wide variety of outpatient procedures is described in Chapter II. The prevalence of CPSP one year after outpatient surgery was $15.3 \%$. Severe CPSP was reported by $3.2 \%$ of patients. The prevalence of CPSP was strongly dependent on the procedure performed. Orthopedic surgery was most often associated with the presence of CPSP. The most important risk factor for the development of CPSP was the presence of preoperative pain. Other risk factors were preoperative analgesic use, surgical fear, lack of optimism, low preoperative quality of life and the presence of acute postsurgical pain.

Chapter III describes the prevalence and predictive factors of CPSP after arthroscopic knee surgery. The prevalence of moderate to severe CPSP was $32.7 \%$. Both the presence of preoperative pain and the preoperative use of analgesics were strongly predictive for the development of CPSP.

The results of a large prospective cohort study, describing hospital-based health care costs during the first year after outpatient surgery, are presented in Chapter IV. Hospital-based health care costs during the first postoperative year were significantly higher in patients with moderate to severe preoperative pain, than in patients without preoperative pain. Furthermore, patients with preoperative pain visited their general practitioner, medical specialist or other health care professional more often with questions about pain and were more often absent from their paid job.

Chapter $\mathrm{V}$ describes the results of a systematic review regarding the genetic risk factors for the development of CPSP after a variety of surgical procedures. Associations with the prevalence or severity of CPSP was reported for polymorphisms in the COMT gene, OPRM1 gene, potassium channel genes, GCH1 gene, CACNG gene, CHRNA6 gene, P2X7R gene, cytokine-associated genes, human leukocyte antigen genes, DRD2 gene and ATXN1 gene. Unfortunately, most studies were small and of retrospective design, and most polymorphisms were described by only one study.

The results of our own clinical study with regard to genetic risk factors for the development of CPSP are presented in Chapter VI. The rs4818 polymorphism within the COMT gene was associated with the prevalence of CPSP three months after 
hysterectomy. No associations were found between the included polymorphisms and CPSP twelve months after hysterectomy. Addition of genetic polymorphisms to the clinical and psychological prediction model for CPSP in this cohort, did not significantly improve the accuracy of the model. 


\section{Samenvatting}

Chronisch postoperatieve pijn (CPOP) is een frequent probleem met potentieel ernstige gevolgen, zoals een verminderde kwaliteit van leven, verzuim van werk en sociale activiteiten, en een toegenomen behoefte aan zorg. Het doel van dit proefschrift was het verbeteren van de kennis ten aanzien van de complexe interactie van klinische, psychologische en genetische risicofactoren voor het ontwikkelen van CPOP na verschillende chirurgische ingrepen.

De resultaten van een grote prospectieve cohort studie, waarin werd gezocht naar de prevalentie en predictoren voor CPOP na verschillende procedures in dagbehandeling, worden beschreven in Hoofdstuk II. De prevalentie van CPOP een jaar na chirurgie in dagbehandeling was 15,3\%. Ernstige CPOP werd gerapporteerd door 3,2\% van de patiënten. De prevalentie van CPOP was sterk afhankelijk van de uitgevoerde ingreep. Orthopedische ingrepen werden het vaakste geassocieerd met de aanwezig van CPOP. De belangrijkste risicofactor voor het ontwikkelen van CPOP was de aanwezig van preoperatieve pijn. Andere risicofactoren waren analgeticagebruik, angst voor de operatie, een gebrek aan optimisme, lage preoperatieve kwaliteit van leven, en de aanwezigheid van acute postoperatieve pijn.

Hoofdstuk III beschrijft de prevalentie en predictoren voor CPOP na arthroscopie van de knie. De prevalentie van matige tot ernstige CPOP was $32,7 \%$. De aanwezigheid van preoperatieve pijn en het gebruik van analgetica in de preoperatieve fase, waren beide sterke predictoren voor de ontwikkeling van CPOP.

De resultaten van een grote prospectieve cohort studie, waarin werd gekeken naar de ziekenhuiskosten in het eerste jaar na chirurgie in dagbehandeling, worden beschreven in Hoofdstuk IV. De ziekenhuiskosten in het eerste postoperatieve jaar van patiënten met matige tot ernstige preoperatieve pijn waren significant hoger dan van patiënten zonder preoperatieve pijn. Patiënten met preoperatieve pijn brachten vaker een bezoek aan hun huisarts, medisch specialist of andere zorgverlener met vragen over pijn, en tevens was het ziekteverzuim hoger.

Hoofdstuk $V$ beschrijft de resultaten van een systematische review omtrent de genetische factoren voor het ontwikkelen van CPOP na verschillende chirurgische ingrepen. Een relatie met de prevalente of ernst van de CPOP werd gerapporteerd voor het COMT gen, OPRM1 gen, kaliumkanaalgenen, GCH1 gen, CACNG gen, CHRNA6 gen, P2X7R gen, cytokine-geassocieerde genen, HAL-genen, DRD2 gen en het ATXN1 gen. Helaas waren de meeste studies klein of retrospectief en werden de meeste polymorfismen maar door een enkele studie beschreven. 
De resultaten van onze eigen klinische studie met betrekking tot de genetische risicofactoren voor het ontwikkelen van CPOP worden beschreven in Hoofdstuk VI. Het rs4818 polymorfisme in het COMT gen was geassocieerd met de prevalentie van CPOP drie maanden na een hysterectomie. Er werd geen relatie gevonden tussen de onderzochte polymorfismen en CPOP twaalf maanden na een hysterectomie. Het toevoegen van genetische polymorfismen aan het bestaande klinische en psychologische predictiemodel voor CPOP in dit cohort, verbeterde de nauwkeurig van het model niet significant. 
Chapter

\section{Valorisation addendum}





\section{Valorisation addendum}

The goal of this thesis is to contribute to the scientific knowledge and understanding of the pathologic processes involved in the human body with regard to pain; but also to find approaches to improve and optimize healthcare in the perioperative setting in order to decrease the prevalence and severity of chronic postsurgical pain (CPSP). The goal of this paragraph is to highlight the societal and economic importance of the research aims in this thesis; both on the voluminous group of surgical patients, as well as on society in general.

We will start by explaining the impact of pain on the individual patient and on society. Second, we will explain the importance of developing prediction models for CPSP. We will end this paragraph by highlighting the future research directions that are necessary to develop methods for preventing CPSP as adequately as possible.

\section{Personal and societal impact of pain}

Pain is an important and widely prevalent health problem, especially in the developed world. Pain causes discomfort and suffering, and research has demonstrated that pain severity is an important determinant of quality of life. ${ }^{1-3}$ Aiming to prevent and treat pain as adequately as possible therefore has a substantial moral ground, and is considered an essential part of optimal patient care. ${ }^{4}$ In addition to this, research has also demonstrated that pain, especially acute postsurgical pain (APSP), is associated with increased morbidity and mortality. Patients who suffer from APSP are prone to cardiac morbidity (e.g. myocardial infarction), pulmonary morbidity (e.g. pulmonary infections), stroke and postoperative delirium. ${ }^{5-7}$ It has also been demonstrated that pain has a negative impact on other organ systems, such as the gastrointestinal system, the renal system, blood coagulation and the immune system. ${ }^{8}$

In addition to the negative consequences of pain for the individual patient, research has shown that pain can have an important negative impact on society in general. Multiple studies have shown that chronic pain is associated with increased consumption of health care resources, absenteeism from work and a substantial associated economic burden. ${ }^{9-13}$ More specifically, moderate to severe acute postsurgical pain has been clearly demonstrated to be associated with delayed hospital discharge and, in case of ambulatory surgery, with unanticipated hospital admission and contact with the general practitioner or other health care professionals. ${ }^{5,14}$ Since it is estimated that over $80 \%$ of all surgical patients experience inadequately managed pain during the acute postoperative phase, it is easy to imagine that APSP leads to a substantial increase in health care costs and a negative impact on society. ${ }^{5}$ This is especially true for societies which are based on a system of solidarity, and in which health care costs are thus considered a shared individual and governmental responsibility. 
The societal consequences of CPSP have been less extensively studied. A small study has demonstrated that patients with severe neuropathic CPSP or neuropathic posttrauma pain visit physicians more frequently and use more prescription medication than patients with less severe pain. ${ }^{15}$ Another study demonstrated an increase in health care utilization of patients with CPSP after kidney transplantation. ${ }^{16}$

The described negative consequences of acute and chronic postsurgical pain on the individual patient (e.g. discomfort and suffering, increased morbidity and mortality) and on society in general (e.g. increased health care costs, absenteeism from work and increased economic burden) are important reasons to make every attempt to reduce the prevalence and severity of APSP and CPSP. As mentioned before, adequate treatment of APSP and CPSP is very important in order to reduce the prevalence and severity of these pain problems, and should be part of optimal patient care. However, prevention will be far more effective in reducing the negative consequences for the individual patient and society. In order to prevent the development of CPSP in postsurgical patients, it is of paramount importance that we first understand the risk factors for the development of CPSP. Only then will we be able to focus research on identifying strategies to prevent the development of moderate to severe CPSP.

\section{Importance of prediction of CPSP}

During the last decade many researchers have attempted to develop tools to identify patients at risk for the development of moderate to severe CPSP. Until recently, the focus has been on demographic, clinical and psychological risk factors. These risk factors can be easily identified with the use of questionnaires and do not require invasive testing. One of the most important risk factors seems to be the presence of moderate to severe preoperative pain. ${ }^{17-20}$ Other risk factors are the presence of moderate to severe acute postoperative pain, preoperative analgesic use, surgical fear, lack of optimism and pain catastrophizing. 2, 17, 21, 22 However, clinical and psychological risk factors have only been able to explain part of the observed variance in the prevalence and severity of CPSP. For this reason, genetic factors have recently become of interest. Our own studies, as well as several others, have been able to identify genetic polymorphisms as possible predictors for CPSP. ${ }^{23,24}$ Especially polymorphisms within the COMT gene, opioid receptor genes, potassium channel genes, GCH1, CACNG, CHRNA6, P2X7R, cytokine-associated genes, human leukocyte antigens, DRD2 and ATXN1 seem to be promising predictors for CPSP.

Defining the risk factors for CPSP is the first step towards reduction and prevention of CPSP. It will create possibilities for an individual approach, which is paramount in the time of personalized medicine. It could help identify the predictors for CPSP on an individual level during the preoperative phase, after which a personalized plan can be made. It will allow patients to be informed of their individual risk at CPSP, which might lead to an informed decision of the treatment plan. In some cases patients might decide 
not to undergo the planned surgery at all. This could be the case for patients with a high risk at developing CPSP, who are about to undergo surgery that is not strictly necessary (e.g. cosmetic procedures), or procedures for which non-surgical alternatives exist. In other cases, a preoperative tailor-made treatment plan might be offered to the patient, in order to try to influence the present risk factors (e.g. treatment of preoperative pain or psychological factors).

Future research will hopefully make us gain even more insight into the predictors for the development of CPSP. In order to further clarify this, future research should not only focus on the intensity of the reported pain (e.g. NRS or VAS), but also on the characteristics of the pain (e.g. neuropathic, inflammatory, etc.), as well as the location of the reported pain. This is important because various types of pain can have a different origin and can therefore be associated with various risk profiles. Future research should also try to further reveal the genetic risk factors for the development of CPSP. Most studies have so far focused on genetic polymorphisms that have been associated with other types of pain, as well as psychiatric diseases. This type of genetic research is also known as hypothesis-driven research. A different approach, in the form of genome-wide associated studies (GWAS) will hopefully lead to the identification of other promising polymorphisms associated with CPSP. ${ }^{25,}{ }^{26}$ Ones GWAS have identified promising polymorphisms, and these data have been validated in large patient cohorts, these polymorphisms can be tested in smaller procedure-specific cohorts, and can be incorporated into clinical and psychological prediction models.

\section{Towards prevention of CPSP}

Our research has demonstrated that the prevalence of CPSP is at least $10-15 \%$ after several types of surgical procedures. ${ }^{17,24,27}$ Several studies have demonstrated that the presence of CPSP is associated with an increase in health care costs and societal economic burden. ${ }^{15,16}$ Our own research found an association between preoperative pain, one of the most important predictors of CPSP, and the amount of hospital-based health care costs as well as absenteeism from paid work. ${ }^{28}$ All these data suggest that adequate prevention of moderate to severe CPSP will be able to reduce health care costs and societal economic burden significantly.

Even though the research in this thesis does not describe preventative methods with regards to CPSP, the importance of adequate prevention in society does warrant a short summary of possible methods for prevention. Future research will have to demonstrate whether these methods will actually be able to reduce the prevalence and severity of CPSP.

Small studies have already suggested that the prevalence of CPSP can be reduced by treating acute postoperative wound pain and neuropathic pain. ${ }^{29,30}$ However, our own studies, as well as several others, have demonstrated that preoperative pain appears to be an even stronger predictor for the development of CPSP. ${ }^{17,18,20}$ It would therefore be 


\section{Chapter 9}

interesting to know if treatment of preoperative pain is able to reduce the prevalence and severity of CPSP even further. In order to be successful, treatment of preoperative pain will probably have to be as timely and as effective as possible, in order to limit preoperative sensitization of the peripheral and central nervous system.

Furthermore, research has also demonstrated that preoperative anxiety, pain catastrophizing and pessimism are important risk factors in the development of CPSP. ${ }^{2}$, 17, 21, 22 The role of targeting these psychological risk factors should also be clarified by future research. More research in the field of genetics and CPSP might lead to new perioperative pharmacologic targets for the prevention and treatment of CPSP.

\section{Summary}

Defining the predictive factors for the development of CPSP after several types of surgery is the first step towards the prevention of CPSP. A decreased prevalence and severity of CPSP will in turn lead to a reduction of health care costs, work absenteeism and societal economic burden. 


\section{References}

1. van Agt HM, Essink-Bot ML, Krabbe PF, Bonsel GJ. Test-retest reliability of health state valuations collected with the EuroQol questionnaire. Soc Sci Med 1994; 39(11): 1537-44.

2. Peters ML, Sommer M, de Rijke JM, et al. Somatic and psychologic predictors of long-term unfavorable outcome after surgical intervention. Ann Surg 2007; 245(3): 487-94.

3. Stessel B, Fiddelers AA, Joosten EA, Hoofwijk DM, Gramke HF, Buhre WF. Prevalence and Predictors of Quality of Recovery at Home After Day Surgery. Medicine 2015; 94(39): e1553.

4. Inspectie voor de gezondheidszorg I. Basisset Medisch Specialistische Zorg Kwaliteitsindicatoren. 2017. https://http://www.igj.nl/documenten/indicatorensets/2016/08/08/basisset-medisch-specialistischezorg-2017.

5. Gan TJ. Poorly controlled postoperative pain: prevalence, consequences, and prevention. J Pain Res 2017; 10: 2287-98.

6. Kalkman CJ, Visser K, Moen J, Bonsel GJ, Grobbee DE, Moons KG. Preoperative prediction of severe postoperative pain. Pain 2003; 105(3): 415-23.

7. Ballantyne JC, Carr DB, deFerranti S, et al. The comparative effects of postoperative analgesic therapies on pulmonary outcome: cumulative meta-analyses of randomized, controlled trials. Anesth Analg 1998; 86(3): 598-612.

8. Joshi GP, Ogunnaike BO. Consequences of inadequate postoperative pain relief and chronic persistent postoperative pain. Anesthesiol Clin North America 2005; 23(1): 21-36.

9. Schaefer C, Sadosky A, Mann R, et al. Pain severity and the economic burden of neuropathic pain in the United States: BEAT Neuropathic Pain Observational Study. ClinicoEconomics and outcomes research : CEOR 2014; 6: 483-96.

10. Nickel R, Raspe HH. [Chronic pain: epidemiology and health care utilization]. Der Nervenarzt 2001; 72(12): 897-906.

11. Breivik H, Collett B, Ventafridda V, Cohen R, Gallacher D. Survey of chronic pain in Europe: prevalence, impact on daily life, and treatment. Eur J Pain 2006; 10(4): 287-333.

12. Liedgens $\mathrm{H}$, Obradovic M, De Courcy J, Holbrook T, Jakubanis R. A burden of illness study for neuropathic pain in Europe. ClinicoEconomics and outcomes research : CEOR 2016; 8: 113-26.

13. Juniper M, Le TK, Mladsi D. The epidemiology, economic burden, and pharmacological treatment of chronic low back pain in France, Germany, Italy, Spain and the UK: a literature-based review. Expert opinion on pharmacotherapy 2009; 10(16): 2581-92.

14. Pavlin DJ, Chen C, Penaloza DA, Polissar NL, Buckley FP. Pain as a factor complicating recovery and discharge after ambulatory surgery. Anesth Analg 2002; 95(3): 627-34.

15. Parsons B, Schaefer C, Mann R, et al. Economic and humanistic burden of post-trauma and post-surgical neuropathic pain among adults in the United States. J Pain Res 2013; 6: 459-69.

16. Hollisaaz MT, Noorbala MH, Irani N, et al. Severity of chronic pain affects health care utilization after kidney transplantation. Transplant Proc 2007; 39(4): 1122-5.

17. Hoofwijk DM, Fiddelers AA, Peters ML, et al. Prevalence and Predictive Factors of Chronic Postsurgical Pain and Poor Global Recovery One Year after Outpatient Surgery. Clin J Pain 2015.

18. Fletcher D, Stamer UM, Pogatzki-Zahn E, et al. Chronic postsurgical pain in Europe: An observational study. Eur J Anaesthesiol 2015; 32(10): 725-34.

19. Macrae WA. Chronic pain after surgery. Br J Anaesth 2001; 87(1): 88-98.

20. Aasvang EK, Gmaehle E, Hansen JB, et al. Predictive risk factors for persistent postherniotomy pain. Anesthesiology 2010; 112(4): 957-69.

21. Theunissen HM, Peters ML, Bruce J, Gramke HF, Marcus MA. Preoperative Anxiety and Catastrophizing: A Systematic Review and Meta-analysis of the Association With Chronic Postsurgical Pain. Clin J Pain 2012; 28(9): 819-41. 
22. Theunissen M, Peters ML, Schepers J, et al. Recovery 3 and 12 months after hysterectomy: epidemiology and predictors of chronic pain, physical functioning, and global surgical recovery. Medicine 2016; 95(26): e3980.

23. Hoofwijk DM, van Reij RR, Rutten BP, Kenis G, Buhre WF, Joosten EA. Genetic polymorphisms and their association with the prevalence and severity of chronic postsurgical pain: a systematic review. $\mathrm{Br} J$ Anaesth 2016; 117(6): 708-19.

24. Hoofwijk DM, van Reij RR, Rutten BP, et al. Genetic polymorphisms and prediction of chronic postsurgical pain 3 and 12 months after hysterectomy. Submitted.

25. Kraft P, Cox DG. Study designs for genome-wide association studies. Advances in genetics 2008; 60: 465504.

26. Geschwind DH, Flint J. Genetics and genomics of psychiatric disease. Science 2015; 349(6255): $1489-94$.

27. Hoofwijk DM, Fiddelers AA, Emans PJ, et al. Prevalence and Predictive Factors of Chronic Postsurgical Pain and Global Surgical Recovery 1 Year After Outpatient Knee Arthroscopy: A Prospective Cohort Study. Medicine 2015; 94(45): e2017.

28. Hoofwijk DM, Fiddelers AA, Stessel B, et al. Severity of preoperative pain is associated with the amount of postoperative hospital-based health care costs during the first year after outpatient surgery. Submitted.

29. Bouman EA, Theunissen M, Bons SA, et al. Reduced incidence of chronic postsurgical pain after epidural analgesia for abdominal surgery. Pain practice : the official journal of World Institute of Pain 2014; 14(2): E76-84.

30. Clarke H, Bonin RP, Orser BA, Englesakis M, Wijeysundera DN, Katz J. The prevention of chronic postsurgical pain using gabapentin and pregabalin: a combined systematic review and meta-analysis. Anesth Analg 2012; 115(2): 428-42. 


\section{Chapter}

\section{Acknowledgements}

Dankwoord 



\section{Dankwoord}

Bijna negen jaar geleden begon ik, destijds als semi-arts, met het verzamelen van data voor de studie die uiteindelijk een belangrijk onderdeel zou worden van mijn proefschrift. Nu, zoveel jaren later, kan ik eindelijk zeggen dat het proefschrift af is en dat ik met veel plezier terugkijk op deze periode. Het was niet altijd makkelijk naast mijn opleiding tot anesthesioloog en later mijn klinische werkzaamheden als staflid. Er zijn meerdere momenten geweest waarop ik mij afvroeg of ik nog de energie had en of het überhaupt zou gaan lukken om het promotietraject tot een goed einde te brengen, maar dit proefschrift is het bewijs dat de aanhouder wint.

Als ik iets heb geleerd, is het dat het verrichten van goed en waardevol wetenschappelijk onderzoek iets is wat geen enkele onderzoeker of promovendus alleen kan. De steun en kritische blik van de mede-onderzoekers en het promotieteam zijn hierbij onmisbaar, maar ook het begrip en de waardering van de collega's op de werkvloer zijn niet te onderschatten. Last, but not least, zonder de steun en het geduld van mijn familie en vrienden zou dit zeker allemaal niet gelukt zijn!

\section{Het promotieteam}

Prof. Dr. Buhre, beste Wolfgang, in 2013 heb je zonder aarzelen de taken van Marco Marcus overgenomen. Je hebt je verdiept in mijn onderzoek, waardoor het voor mij mogelijk was door te gaan met mijn promotietraject. Je bent zeer nauw betrokken geweest bij de vier manuscripten die nog moesten worden geschreven en je wist mij in contact te brengen met deskundigen van andere vakgebieden. Ik wil je bedanken voor je vertrouwen en je onmisbare hulp om dit promotie-traject tot een goed einde te brengen.

Prof. Dr. Joosten, beste Bert, zonder jouw hulp had ik dit proefschrift nooit tot een goed einde kunnen brengen. Ondanks al het extra werk en tijd, had je er vertrouwen in dat ik in staat was het project 'genetica en pijn' op te pakken en ben je me hierin blijven steunen. Hierdoor heb ik veel ervaring kunnen opdoen als onderzoeker en dit heeft mijn proefschrift vele malen interessanter gemaakt. Je kritische blik en je snelle reacties op mijn vragen waren onmisbaar voor dit proefschrift!

Dr. Gramke, beste Hans, jouw werk was de basis waaruit al mijn onderzoek is voortgekomen. Als geen ander had je inzicht in de stof en ontelbare keren heb ik jouw proefschrift gelezen om inspiratie op te doen voor mijn eigen manuscripten. Ondanks al je andere verplichtingen en drukke bestaan wist je tijd te maken om mijn manuscripten te lezen en te verbeteren door je kritische blik.

Dr. Fiddelers, beste Audrey. Vanaf het begin was jij mijn steun en toeverlaat. Als geen ander wist jij hoe de database in elkaar stak en heb je mij de beginselen van het 
verrichten van wetenschappelijk onderzoek bijgebracht. Je hebt me geleerd een database op te bouwen en de data te analyseren. Bij elke vraag die ik had, was jij bereid om mij te helpen, ongeacht hoe veel tijd je dit zelf kostte. Zelfs nadat je ging werken voor een andere afdeling ben jij betrokken gebleven en was je altijd bereid met een kritische blik naar mijn manuscripten te kijken. Jij was de rode draad in dit promotietraject!

\section{De co-auteurs}

Prof. Dr. Marcus, beste Marco, jij was de eerste die wetenschappelijke potentie in mij zag. Je hebt mij, al heel vroeg in mijn carrière, het vertrouwen geschonken om met deze database aan de slag te gaan en dit onderzoek uit te bouwen tot een mooi project. De eerste jaren van mijn promotie hebben we dan ook samen gewerkt aan een aantal mooie publicaties. Deze jaren hebben mij gevormd!

Dr. Maurice Theunissen, al vanaf het begin was jij, samen met Audrey, betrokken bij mijn onderzoek en was je altijd bereid methodologische vragen te beantwoorden, ook als je zelf druk bezig was. Veel dank ben ik je verschuldigd voor het beschikbaar stellen van de perfect uitgebouwde database met hysterectomiepatiënten, en voor je bereidheid om bij herhaling toe te lichten waar bepaalde informatie te vinden was.

Drs. Roel van Reij, zonder jou was het nooit gelukt om de genetische database uit te bouwen. Samen met Dr. Nynke van den Hoogen heb je me geholpen met het klaarmaken van alle samples voor genetische analyse. Je heb je, met behulp van collega's in Bonn, weten te bekwamen in de ontzettend moeilijk stof van de statische analyse van GWAS-data. Je was onmisbaar bij het klaarmaken van de database en hulp bij de analyses. Ik wens je heel veel succes bij het schrijven van je eigen proefschrift!

Dr. Bjorn Stessel, jarenlang hebben we in hetzelfde schuitje gezeten. We zijn min of meer samen aan ons promotietraject begonnen en deelden interesse in hetzelfde onderwerp. Ik heb veel gehad aan je goede ideeën en aan je kritische blik. Jij hebt je verdediging er inmiddels al opzitten en ik hoop dat ik je snel mag volgen!

Prof. Dr. Bart Rutten en Dr. Gunter Kenis, jullie hebben me wegwijs weten te maken in de beginselen van het genetisch onderzoek. Jullie ervaring en geduld heeft ertoe geleid dat ik wist waar ik moest beginnen!

Prof. Dr. Carmen Dirksen, bedankt voor je tips en adviezen met betrekking tot de ingewikkelde stof van de economische analyses.

Dr. Pieter Emans, dankzij jouw hulp was ik in staat de resultaten met betrekking tot chronisch postchirurgische pijn in een cohort van orthopedische patiënten op de juiste manier te interpreteren. 
Dr. Sander van Kuijk, bedankt voor jouw hulp, vaak op zeer korte termijn, met methodologische en statistische vraagstukken en voor je kritische blik op de geschreven manuscripten.

\section{Overige collegae}

Hermina Gielen, jou ben ik speciale dank verschuldigd dat je altijd bereid was een plekje te zoeken in de drukke agenda van Wolfgang, zodat mijn proefschrift geen vertraging zou oplopen. Ook bedank ik je voor de praktische hulp in de afrondende fase en de tips en informatie die ik nodig had om alles tijdig af te hebben.

Mijn dank gaat uit naar de leden van de beoordelingscommissie; Prof. Dr. Karin Faber als voorzitter, Prof. Dr. Ide Heyligers, Prof. Dr. Roy Kruitwagen, Prof. Dr. Gert Jan Scheffer en Dr. Marc Buise. Bedankt voor jullie beoordeling en goedkeuring van mijn proefschrift.

Alle AIOS en semi-artsen die hebben meegeholpen met het verzamelen van de data voor mijn onderzoek mogen natuurlijk niet vergeten worden. Zonder de vele uren die jullie hebben besteed aan het includeren van patiënten en het bijhouden van data in de database, was mijn onderzoek nooit mogelijk geweest.

Beste maten, vakgroepleden uit het Zuyderland, bedankt voor het vertrouwen dat jullie me hebben gegeven door mij in jullie vakgroep op te nemen! Jullie hebben geen idee hoeveel ik jullie steun heb gewaardeerd en hoe blij ik ben met jullie voorbereidingen om massaal aanwezig te kunnen zijn bij de verdediging van mijn proefschrift.

Mijn paranimfen Fleur Franssen en Markus Janssen, jullie wil ik bedanken voor jullie onmisbare hulp om deze dag tot een goed einde te brengen!

\section{Mijn familie}

Pap, jij was de eerste die tegen me zei dat ik deze kans niet moest laten schieten, de eerste die me ervan wist te overtuigen dat ik dit kon. Zonder jouw steun was ik nooit aan dit promotietraject begonnen. Marijke, jij hebt er altijd voor gezorgd dat álle kinderen konden rekening op een veilige plek en de nodige steun!

Jos en Philomène, bedankt voor alle keren, gepland en ongepland, dat jullie op Lexie hebben willen passen. Hierdoor heb ik de tijd gekregen om in alle rust aan mijn onderzoek te werken én hebben Robbie en ik de tijd gekregen om regelmatig samen lekker te gaan eten.

Lieve Robbie, toen we elkaar leerden kennen was ik al druk bezig met mijn promotietraject, waardoor ik ook regelmatig thuis in mijn eigen tijd bezig was. Dit heeft je er niet van weerhouden om me jarenlang te steunen. Meerdere keren heb ik eraan 
gedacht de moed op te geven, maar jij wist me er steeds van te overtuigen dat ik in staat was dit tot een goed einde te brengen, waardoor ik weer voldoende moed kreeg om door te zetten. Inmiddels zijn we ruim zes jaar verder, zijn we getrouwd, hebben we een prachtige dochter, Lexie, en is ons tweede meisje onderweg. Ik hou ontzettend veel van jullie en kijk ernaar uit om nog meer tijd met jullie door te brengen! 
Chapter

Curriculum Vitae and list of publications 



\section{Curriculum vitae}

Daisy Hoofwijk was born on July $31^{\text {st }}, 1985$ in Sittard, The Netherlands. After graduating cum laude from high school at the Trevianum in Sittard in 2004, she started medical training at the University of Maastricht, where she obtained her bachelor degree cum laude in 2007 and her master degree in 2010. She finished her scientific internship at the department of Anesthesiology and Pain Treatment of the Maastricht University Medical Center, during which she started gathering the data for her thesis. After receiving her medical degree in 2010 she started her residency in Anesthesiology at the Maastricht University Medical Center. She finished her residency in 2016, after which she started working as a staff anesthesiologist and followed a fellowship in Pain Treatment in the same hospital. In 2017 she started working as an anesthesiologist and pain practitioner in Zuyderland Medical Center, Heerlen and Sittard. Daisy is married to Robbie, and together they have a daughter, Lexie. 


\section{List of publications}

\section{Publications}

- Hoofwijk DMN, Fiddelers AAA, Peters ML, Stessel B, Kessels AG, Joosten EA, Gramke $\mathrm{HF}$, Marcus MAE. Prevalence and predictive factors of chronic postsurgical pain and poor global recovery one year after outpatient surgery. Clin J Pain, 2015 Dec;31(12):1017-25

- Stessel B, Fiddelers AAA, Joosten EA, Hoofwijk DMN, Gramke HF, Buhre WFFA. Prevalence and predictors of quality of recovery at home after day surgery. Medicine 2015 Sep;94(39):e1553

- Hoofwijk DMN, Fiddelers AAA, Emans PJ, Joosten EA, Gramke HF, Marcus MAE, Buhre WFFA. Prevalence and predictive factors of chronic postsurgical pain and poor global recovery one year after outpatient knee arthroscopy; a prospective cohort study. Medicine 2015 Nov; 94 (45): e2017

- Hoofwijk DMN, van Reij RR, Rutten BP, Buhre WF, Joosten EA. Genetic polymorphisms and their association with the prevalence and severity of chronic postsurgical pain: a systematic review. Br J Anaesth 2016 Dec;117(6):708-719

- Stessel B, Theunissen M, Marcus MA, Joosten EA, van Kuijk SMJ, Fiddelers AAA, Peters ML, Hoofwijk DMN, Buhre WFFA, Gramke HF. Prevalence and predictors of patient non-adherence to pharmacological acute pain therapy at home after day surgery: a prospective cohort study. Pain Pract 2018 Feb;18(2):194-204

- Hoofwijk DMN, Fiddelers AAA, Stessel B, Gramke HF, Dirksen CD, van Kuijk SMJ, Joosten EA, Buhre WF. Severity of preoperative pain is associated with the amount of postoperative hospital-based health care costs during the first year after outpatient surgery. Submitted

- Hoofwijk DMN, van Reij RRI, Rutten BPF, Kenis G, Theunissen M, Joosten EA, Buhre WF, van den Hoogen NJ. Genetic polymorphisms and prediction of chronic postsurgical pain 3 and 12 months after hysterectomy. Submitted

\section{Book Chapters}

- Chronische pijn na een lumpectomie; Hoofwijk DMN, Fiddelers AAA, Bouman EA. Probleemgeoriënteerd denken in de pijngeneeskunde, De Tijdstroom, 2017 


\section{Posters and presentations}

- Hoofwijk DMN, Fiddelers AAA, Klein Ovink J, Gramke HF, Marcus MAE. Postoperative analgesic use increases by providing a preoperative prescription. Euroanesthesia 2010, Helsinki, Finland

- Fiddelers AAA, Hoofwijk DMN, Klein Ovink J, Gramke HF, Marcus MAE. Preoperative analgesic use is a predictor of postoperative pain in ambulatory surgery. Euroanesthesia 2010, Helsinki, Finland

- Hoofwijk DMN. Does preoperative analgesic use and quality of life predict postoperative pain after outpatient surgery? Presentation NVA dagen, Maastricht, The Netherlands.

- Hoofwijk DMN, Fiddelers AAA, Gramke HF, Marcus MAE. Does preoperative quality of life predict pain after outpatient surgery? Euroanesthesia 2011, Amsterdam, The Netherlands

- Hoofwijk DMN, Fiddelers AAA, Kropman L, Gramke HF, Marcus MAE. Health care consumption during the first four days after outpatient surgery. Euroanesthesia 2012, Paris, France.

- Fiddelers AAA, Hoofwijk DMN, Gramke HF, Marcus MAE. The correlation between preoperative, acute postoperative and persistent postoperative pain. Euroanesthesia 2012, Paris, France

- Hoofwijk DMN, Fiddelers AAA, Gramke HF, Marcus MAE. Health care consumption and chronic postsurgical pain one year after outpatient surgery. Anesthesiology (ASA meeting) 2012, Washington DC, United States

- Hoofwijk DMN, Fiddelers AAA, Gramke HF, Marcus MAE. Does acute postoperative pain truly predict chronic postsurgical pain (CPSP)? $7^{\text {th }}$ World Congress World Institute of Pain, Maastricht, The Netherlands

- Hoofwijk DMN, Fiddelers AAA, Emans PJ, Joosten EA, Gramke HF, Marcus MAE, Buhre WFFA. Prevalence and predictive factors of chronic postsurgical pain and global recovery one year after outpatient knee arthroscopy. Anesthesiolgy (ASA meeting) 2015, San Diego, United States

- Van Reij RRI, Hoofwijk DMN, Rutten BP, Kenis G, van den Hoogen NJ, Buhre WFFA, Joosten EA. Genetic polymorphisms and their association with the prevalence and severity of chronic postsurgical pain. NVA Wetenschapsdag 2016, Ede, The Netherlands 


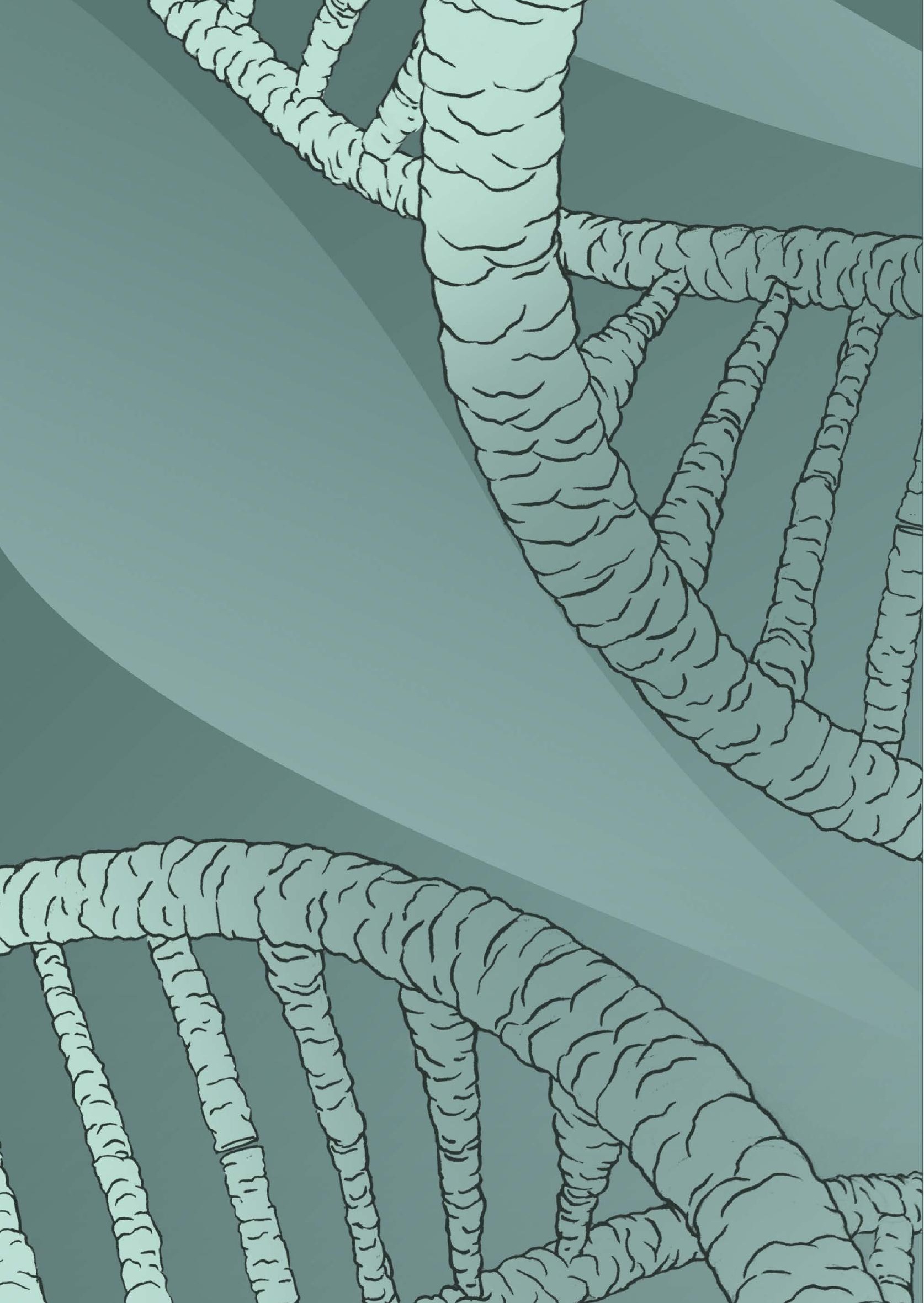

